\title{
Neuroimaging markers of major depressive disorder: orbitomedial prefrontal cortex and beyond
}

Citation for published version (APA):

Samara, Z. (2015). Neuroimaging markers of major depressive disorder: orbitomedial prefrontal cortex and beyond. [Doctoral Thesis, Maastricht University]. Boekenplan. https://doi.org/10.26481/dis.20151106zs

Document status and date:

Published: 01/01/2015

DOI:

10.26481/dis.20151106zs

Document Version:

Publisher's PDF, also known as Version of record

\section{Please check the document version of this publication:}

- A submitted manuscript is the version of the article upon submission and before peer-review. There can be important differences between the submitted version and the official published version of record.

People interested in the research are advised to contact the author for the final version of the publication, or visit the DOI to the publisher's website.

- The final author version and the galley proof are versions of the publication after peer review.

- The final published version features the final layout of the paper including the volume, issue and page numbers.

Link to publication

\footnotetext{
General rights rights.

- You may freely distribute the URL identifying the publication in the public portal. please follow below link for the End User Agreement:

www.umlib.nl/taverne-license

Take down policy

If you believe that this document breaches copyright please contact us at:

repository@maastrichtuniversity.nl

providing details and we will investigate your claim.
}

Copyright and moral rights for the publications made accessible in the public portal are retained by the authors and/or other copyright owners and it is a condition of accessing publications that users recognise and abide by the legal requirements associated with these

- Users may download and print one copy of any publication from the public portal for the purpose of private study or research.

- You may not further distribute the material or use it for any profit-making activity or commercial gain

If the publication is distributed under the terms of Article $25 \mathrm{fa}$ of the Dutch Copyright Act, indicated by the "Taverne" license above, 
Neuroimaging Markers of Major Depressive Disorder: Orbitomedial Prefrontal Cortex and beyond 



\title{
Neuroimaging Markers of Major Depressive Disorder:
}

\section{Orbitomedial Prefrontal Cortex and beyond}

\author{
DISSERTATION \\ to obtain the degree of Doctor at Maastricht University, \\ on the authority of the Rector Magnificus,, Prof. Dr. L.L.G. Soete \\ in accordance with the decision of the Board of Deans, \\ to be defended in public on Friday $6^{\text {th }}$ November 2015 at 10:00 o'clock \\ by \\ Zoe (Zoi) Samara
}

born on the $16^{\text {th }}$ of June 1977 in Thessaloniki, Greece 


\section{Promotor:}

Prof. dr. J.G. Ramaekers (Maastricht University)

Copromotors

Dr. P. Stiers (Maastricht University)

Dr. E.A.T. Evers (Maastricht University)

Assessment Committee:

Prof. dr. W. Riedel (chair; Maastricht University)

Prof. dr. T. van Amelsvoort (Maastricht University)

Prof. dr. R. Goebel (Maastricht University)

Prof. dr. D. Margulies (Max Planck Institute)

Prof. dr. S. Rombouts (Universiteit Leiden)

ㄷ Zoe (Zoi) Samara, Maastricht 2015

Publisher Boekenplan, Maastricht

ISBN 9789086663910

All rights reserved 


\section{Contents}

Chapter 1

Introduction

Chapter 2

Human orbitomedial prefrontal cortex: Intrinsic connectivity parcellation and functional organization

Chapter 3

Orbitomedial prefrontal cortex functional connectivity markers of Major Depression vulnerability and disease

Chapter 4

Altered face processing in Major Depression Vulnerability:

Inhabiting different social worlds?

Chapter 5

Discussion

Summary

Addendum

Knowledge valorization

Curriculum Vitae \& Publications

Acknowledgements 

Chapter 1

Introduction

"What is depression like?" he whispered "It's like drowning. Except you can see everyone around you breathing" 


\section{Background}

\section{Major Depressive Disorder syndrome}

Major Depressive Disorder (MDD) is a psychiatric syndrome affecting $4.7 \%$ of the population worldwide and women twice as often as men (Ferrari et al., 2013). According to DSM-IV TR (APA, 1994), MDD is diagnosed as the experience of episodes of persistent negative mood or anhedonia accompanied by at least four of the following somatic, affective or cognitive symptoms: significant weight changes, sleep disturbances, psychomotor agitation or retardation, fatigue, feelings of worthlessness or excessive guilt, indecisiveness and recurrent suicidal thoughts. The symptoms must be present for a minimum of 2 weeks, represent a marked change from previous functioning and cause significant distress. This definition of MDD emerged from efforts in psychiatry to formulate objective and reliable criteria to guide diagnosis in clinical and research settings. Although these diagnostic criteria are useful in practice, it should be emphasized that MDD is not the negative, pessimistic mood or anhedonic state per se; negative or "down" states are commonly experienced by otherwise healthy people during normal mood variations or in response to stressful experiences and significant life changes. Rather, MDD is better defined as a proclivity or susceptibility of the individual to enter such "down" states, coupled with an inability to successfully upregulate his/her mood and disengage from the downward spiral of physiological and psychological events which subsequently unfolds (Holtzheimer \& Mayberg, 2011).

Subjectively, MDD presents as a negative view of the self, the world and the future (Beck's famous cognitive triad; Beck, 2008) and negative, dysfunctional self-schemata (maladaptive internal representations of ideas or experiences related to one' s self) are at the core of its clinical picture. The average age of MDD onset is around 32 years but MDD occurs also frequently in adolescent or early adulthood (Ferrari et al., 2013). The course of MDD can be episodic or chronic, with or without full symptomatic remission between the episodes, resulting in a quite variable clinical picture in each individual (Figure 1). MDD is associated with significant personal, societal and financial costs for both the sufferer and his/her immediate and extended social circle. It is projected to be the first overall contributor to the worldwide burden of disease in 2030 (Holtzheimer \& Mayberg, 2011). MDD is often comorbid with other mental and physical health conditions (Ferrari et al., 2013), the most impactful of which is the decision of the sufferer to end his/her life, occurring $21 \%$ more often during depressive episodes (Holma et al., 2010). 

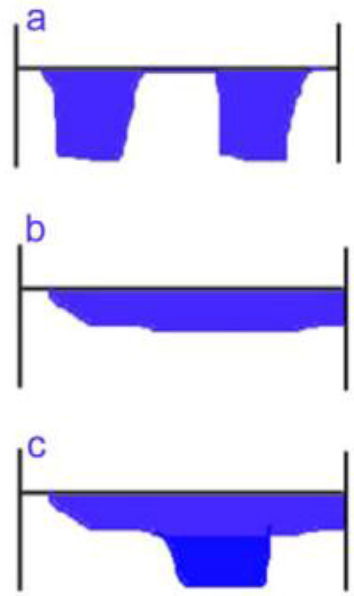
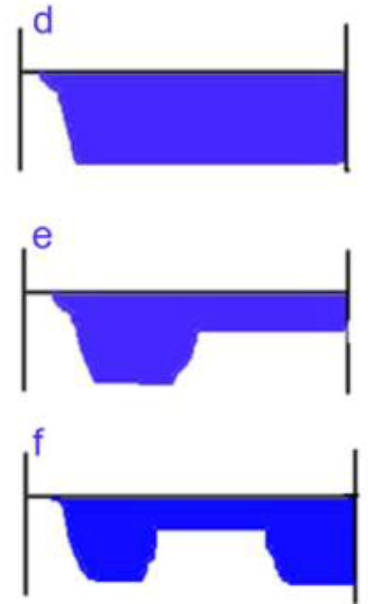

Figure 1. Graphical depiction of the variable course of MDD. The horizontal axis plots the time-course over lifespan and the vertical axis plots clinically significant mood variations. a) Recurrent MDD with 2 episodes, b) dysthymic disorder, c) single MDD episode superimposed on antecedent dysthymic disorder ("double depression"), d) chronic MDD episode, e) MDD episode in partial remission and f) recurrent MDD with 2 episodes (similar to a) but without full inter-episode recovery. Figure originally published in Klein (2010).

Early diagnosis and individually tailored treatment is instrumental for the alleviation of MDD. However, MDD patients frequently remain un- or misdiagnosed long past their first depressive episode (van Rijswijk et al., 2009), getting caught up amidst contradicting evaluations by general practitioners, psychologists, psychiatrists and health staff in general. Even when correctly diagnosed, MDD patients often undergo multiple unsuccessful treatment attempts. First-line MDD treatments are psychotherapy, predominantly cognitive-behavioral interventions and pharmacotherapy with various types of antidepressants. Efficacy rates of MDD treatment are sobering. Initial treatment response to the currently most commonly used antidepressant class, selective serotonin reuptake inhibitors, ranges between $40-60 \%$. Full remission is achieved only by $30-45 \%$ of the treatment responders (Carvalho et al., 2007) while 1/3 of the patients under therapy develops recurrent symptoms (Souery et al., 2006). Given all these, it is common for MDD patients to have tried various kinds of treatments and several combinations of mono- and adjunct therapy. Alternative types of treatment for moderate depression include repetitive transcranial magnetic stimulation (rTMS) of the dorsolateral prefrontal cortex and lifestyle changes such as exercise, light therapy and dietary supplements. Severe, refractory MDD is treated with electroconvulsive therapy (see Figure 2 for the course of 
treatment attempts of MD patients). The efficacy of new treatments such as neuro-feedback therapy, vagus nerve stimulation and deep brain stimulation (DBS) in the subgenual cingulate and other brain regions is currently tested in randomized control trials.

\section{Major Depressive Disorder Treatment Algorithm}

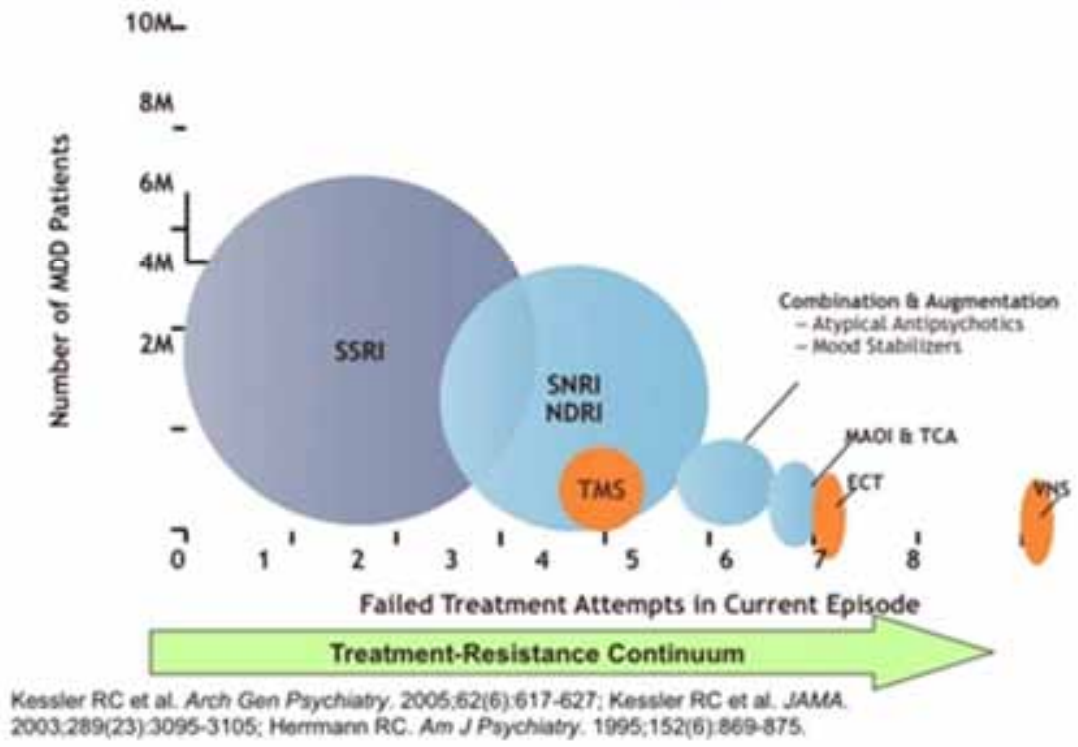

Figure 2. Average course of treatment attempts for MDD. SSRI (Selective Serotonin Reuptake Inhibitor); SNRI (Serotonin Norepinephrine Reuptake Inhibitor); NDRI (Norepinephrine Dopamine Reuptake Inhibitor); MAOI (Mono Amine Oxidase Inhibitor); TCA (Tricyclic Antidepressant); ECT (Electro Convulsive Therapy); VNS (Vagus Nerve Stimulation). Figure originally published in Kessler et al., (2005).

\section{MDD etiology and genetic influences}

The etiology of MDD is multifactorial but it has been established that genetic load influences the development of the syndrome. MDD heritability estimates in monozygotic twins range between $40 \%$ and $50 \%$ (Sullivan et al., 2000) while other first-degree relatives of MDD patients run a $2-5 \%$ higher risk of developing MDD themselves, depending on the severity and lifecourse of their kin's MDD (Weissman et al., 1996). Identifying the genes responsible for MDD heritability is complicated by gene-environment interactions and a complex mode of inheritance in which multiple genes with small effect sizes are likely involved (Lohoff, 2010). Serotonergic genes were 
the first candidate genes for MDD susceptibility given the effects of many antidepressant drugs on the availability of serotonin in the brain. Several studies implicate the serotonin transporter gene (SLC6A4) and its 5-HTTLPR polymorphism in the promoter region of the gene as well as the serotonin receptor gene HTR2A in MDD. Variants of the tryptophan hydroxylase (TPH2) gene, associated with serotonin production, have also been implicated in MDD vulnerability. Recently, due to the recognition of the role of the brain-derived neurotrophic factor (BDNF) in neurogenesis and mood disorders, the association of BDNF's Val66Met polymorphism with MDD has also been set under investigation (Lohoff, 2010).

Immediate relatives of MDD patients are thus genetically predisposed to develop MDD themselves. However, it should be noted that: a) childhood experiences of severe neglect and abuse, recent stressful life events and the quality of core intimate relationships of the individual (Uher, 2008) influence the onset and perpetuation of MDD, and $b$ ) relatives of MDD patients are often under significant burden in the form of experiencing responsibility for the depressed person, loss of control in their everyday life and role-reversals in their familial relationships (Skundberg-Kletthagen et al., 2015). The burden of MDD for the family unit is particularly pronounced for patients' offspring who are both deprived of responsive, functional parents who would fulfill the important parental role of modeling appropriate life-coping strategies and at the same time impacted early in life by the repercussions of parental MDD (i.e. unemployment, marital discord, substance dependence, etc.). Hence, MDD vulnerability when operationalized as "having a first-degree relative with MDD" likely entails epigenetic contributions as well (Hayden et al., 2014) and should be looked at as multidimensional, even though disentangling the genetic from the environmental influences is challenging.

\section{Neuroimaging biomarkers in psychiatry}

MDD is a multifactorial, multidimensional syndrome which does not occur suddenly in the individual's life. Rather, it is the interaction of genetic loads that confer both vulnerability and resilience to mood disorders coupled with the interplay between aggravating and protective environmental influences and the net sum of all these effects at any given point in time. In order to elucidate its etiology and improve our prevention, diagnosis and treatment strategies, it is important to distinguish between predisposing factors, precipitating conditions, manifestations of the current depressive episode and possible adaptation-to-disease or compensation processes. It is also crucial that this distinction is not made at the level of behavioral symptoms, such as the DSM criteria mentioned above, that have been put together to aid classification in practice. Symptoms of psychiatric disorders, similarly to those of somatic conditions, are shared by many conditions and are, thus, non-specific. More importantly, they are merely the behavioral manifestations of disease processes and hence only remotely related to causal factors. In 
the case of MDD this is exemplified in the distinction made in a previous section between the "down" state of the organism during the episode (i.e. the symptoms observed) and the inability to regulate mood states effectively (i.e. one facet of the disorder).

The advent of modern non-invasive neuroimaging techniques has enabled psychiatry to move from measuring self-reported behavioral symptoms to quantifying brain biomarkers, i.e. characteristics that are "objectively measured and evaluated as indicators of normal biological processes, pathogenic processes, or pharmacologic responses to a therapeutic intervention" (Strimbu \& Tavel, 2010). Neuroimaging components, i.e. anatomical properties and functional processes that can be measured with EEG, MEG, PET and MRI tools hold a lot of promise for advancing our understanding of disease processes and proving useful in the clinic. By studying large cohorts of patients, at-risk individuals and healthy controls we can identify components in the brain signal that constitute biomarkers of MDD vulnerability (or trait markers). Vulnerability biomarkers have also been defined as "measureable components unseen by the unaided eye", i.e. endophenotypes (Gottesman \& Gould, 2003) and are suggested to constitute the link between the genetic disease load (genotype) and its behavioral expression (phenotype). According to Gottesman \& Gould (2003) endophenotypes "decompose the psychiatric disease syndrome into straightforward or direct entities which can be successfully linked to the disease's genetic underpinnings". These measurable components constitute endophenotypes if they meet the following criteria: a) are associated with illness in the population, 2) are heritable, 3) manifest in an individual regardless of whether the illness is active or not and 4) within families cosegregate with the illness (Gottesman \& Gould, 2003; Kendler \& McNeale, 2010). Since MDD vulnerability biomarkers represent possible causal factors of the syndrome they can aid in the elucidation of disease mechanisms and gene-disease associations. In samples of patients and controls we can also isolate brain alterations that manifest during depressive episodes, i.e. biomarkers of MDD disease (or state markers) (Figure 3). Disease state biomarkers are brain changes observed specifically during the acute phase of a disorder and are believed to represent the correlates of the behavioral symptoms and/or compensation mechanisms in light of them.

Both types of markers might prove useful in prevention, diagnosis and treatment; at risk individuals could be identified based on brain patterns that confer susceptibility and the modification of these predisposing patterns might become the target for prevention interventions. In patients, diagnosis can be improved by using objective measures from brain scans alongside the subjective report and evaluation of symptoms while treatment could become more effective if focused on reversing the manifestations of the current episode at the first instance. Trait markers, which represent "fixed" changes, 
might be targeted during the remitted phase to help sustain response to treatment and reduce relapse risk.
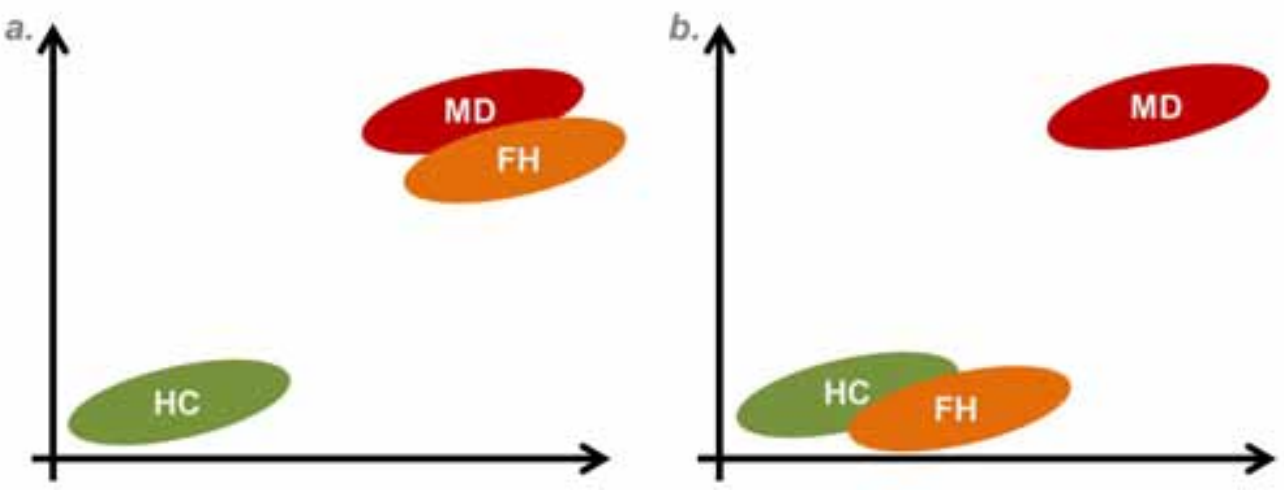

Figure 3. Graphical representation of how the groups of participants are used to delineate trait and state effects. a) In markers of vulnerability (trait markers) alterations or changes are isolated that are common in patients and at-familial-risk individuals, b) In markers of disease (state markers) alterations are unique to patients currently experiencing a depressive episode. MD; major depression participants; $\mathrm{FH}$; family history participants; $\mathrm{HC}$; healthy control participants.

\section{Scope}

\section{What type of biomarkers should we be looking for in MDD?}

Neuroimaging abnormalities in various brain regions have been reported for MDD. Some of the most well documented findings converge on the involvement of the medial prefrontal cortex, mainly the subgenual and rostral cingulate cortex, areas of the medial and lateral orbitofrontal surface and the anterior insula (Mayberg et al., 2005; Pezawas et al., 2005; Price \& Drevets, 2010; Pizzagalli, 2011). These areas, spatially positioned within what is referred to as the orbitomedial prefrontal cortex (OMPFC), together with subcortical affective and autonomic centers, subserve cognitive, emotional and reward processes (see Figure 4 for the limbic-cortical dysregulation model of MDD). Given the presence of widespread abnormalities, MDD is likely not the pathology of a single brain area but rather constitutes a "systems-level" or connectivity disorder, arising from and affecting the pathways of higher-order cortical, limbic and autonomic integration. Evidence for the existence of such circuits comes from post mortem and tracing findings of anatomical connectivity between various OMPFC regions and the amygdala, the hippocampus, the striatum, the thalamus, the hypothalamus and midbrain areas (Price \& Drevets, 2010). Treatments such as ablative neurosurgery, DBS and repetitive TMS with OMPFC targets demonstrate that these circuits are critically involved in MDD. Firstly, the interruption or 
stimulation of white matter tracts in various brain sites (either frontal or limbic) by ablative surgery and DBS leads to symptomatic improvement of severe, treatment-resistant depression (Riva-Posse et al., 2013). Secondly, the antidepressant effect of TMS on left dorsolateral PFC sites is related to the anti-correlation of the site with the subgenual cingulate (Fox et al., 2012).

What is more, it appears that the functional connectivity, i.e. the functionally integrated relationship, between the areas implicated in the MDD affected networks is highly relevant as an MDD biomarker. Although disruption of white matter tracts, or structural connectivity abnormalities are likely also part of the MDD syndrome, especially in severe MDD, functional connectivity might prove more useful in therapeutic applications. In some cases, as soon as DBS contacts in the subgenual cingulate become active, patients report that the subjective feeling of depressed mood vanishes. Therefore, simply modulating the function of the network alone, in the absence of long-term anatomical changes, produces antidepressant effects. Thus, an appropriate aim for improving therapeutic interventions might be to optimize non-invasive neuro-modulation treatments in order to effect the necessary and sufficient functional connectivity changes for initial response to treatment. The resulting modulated function of the network, will, at the long term, reverse or shape the essential structural pathways for sustained treatment response and eventually remission.

Another type of biomarker that holds promise for use in MDD diagnosis and treatment is the response of brain regions during the presentation of human faces, either neutral or with emotional expressions. Human faces are biologically and socially engraved primary reinforcers and face processing is instrumental in any human interaction. Face processing is complex: it involves processing the face as a visual stimulus, recognition of the face's identity (familiar face or not) since each face is a unique stimulus and processing of the face's emotional expression together with its meaning for the social interaction and significance for the individual. Thus, face processing involves self-referential, emotional and social processing subserved by multiple core neurobiological systems (Stuhrmann et al., 2011). Brain abnormalities in processing of neutral and emotional faces are central to MDD. Neuroimaging findings have repeatedly implicated the amygdala, the OFC, the fusiform gyrus as well as striatal and other subcortical areas in altered facial processing in MDD patients. Face perception activations have also been found to differentiate between unipolar and bipolar depression with high accuracy (Mourão-Miranda et al., 2012; Grotegerd et al., 2014), a feature that can prove invaluable for differential diagnostics in clinical settings. 


\section{Limbic-Cortical Dysregulation Model}

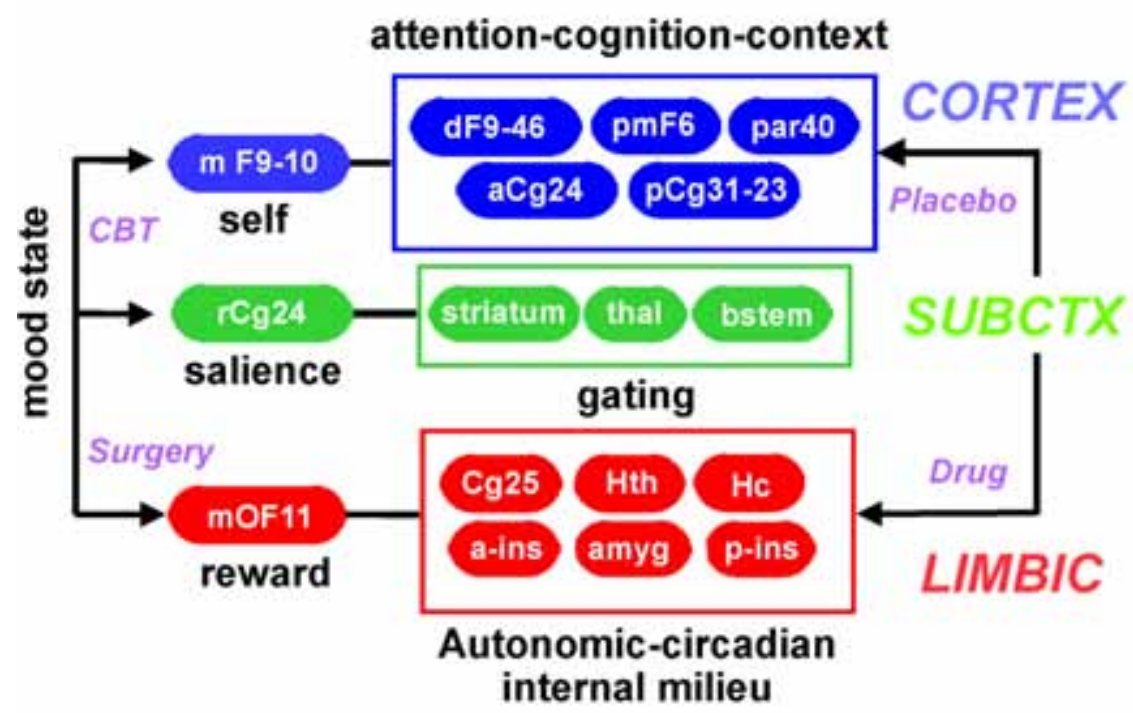

Figure 4. The limbic-cortical dysregulation model of MDD, based on PET data, describes alterations in several areas within the OMPFC. The early observations that several brain regions show abnormal metabolism in depression lead to the proposition that MDD is a systems-level or "connectivity disorder". Figure originally published in Mayberg (2003).

\section{Which tools to use to study MDD biomarkers?}

Magnetic Resonance Imaging-based tools have become invaluable in the study of patient populations. Mapping brain activity with functional MRI (fMRI) is a non-invasive technique that does not involve contrast agents, is fairly fast and enables neuronal source localization. fMRI records the BloodOxygenated-Level-Dependent (BOLD) MR signal, an indirect measure of local oxygen consumption that is based on the MR signal distortion induced by the magnetic properties of oxyhemoglobin in the blood. Although it is a sluggish and indirect measure of neuronal activity detected over various sources of noise, BOLD signal substantially reflects brain activity (Logothetis et al., 2001; Huettel et al., 2004) during simple and complex tasks with variable paradigms (Goebel, 2007). Block, event-related and rapid eventrelated fMRI designs are used to study process-specific brain mechanisms across groups under controlled task conditions. In the current thesis we use BOLD activations to identify MDD-specific biomarkers during face processing.

Soon after BOLD activations had been explored during various experimental paradigms, neuroscientists noticed that certain brain regions were consistently deactivated across a variety of goal-directed tasks 
(Fransson, 2005). Based on these observations and evidence from PET studies on cerebral blood flow and oxygen consumption, Raichle et al. (2001) put forward that during awake rest an organized "default" mode of brain function can be observed which is attenuated during tasks. This proposition turned the focus on the "spontaneous" brain activity observed under unconstrained mental states between brain regions that are functionally connected. Resting-state functional brain networks are studied with MRI using the low frequency $(<0.1 \mathrm{~Hz})$ fluctuations in the BOLD signal (Biswal et al., 1997) across periods during which participants are instructed to not engage in specific mental tasks. Low frequency BOLD co-fluctuations (i.e. correlations) can be calculated between proximal (short-range) and distal (long-range) regions, capturing their functional connectivity or synergy. The functional coupling between any two brain areas measured in this way reflects both direct routes, afferent or efferent anatomical connections between the regions, and indirect links, modulations via a third region. Resting-state MRI functional connectivity putatively reveals a basic configuration of functional pathways that shapes the coordinated response of the regions in a network in response to environmental demands.

As mentioned above, the functional connectivity within and of the OMPFC with distal brain areas is highly relevant in MDD. However, examining the functional connectivity of an extended brain region such as the OMPFC in a systematic and un-biased way is not a trivial problem in neuroscience. The current paradigm of cortical functional organization comes from cytoarchitectonic and tracing studies which demonstrate that the cortex is neither structurally nor functionally homogenous. The cortical surface is made up from assemblies of neurons distinguished by their type, density and organization in layers. These structurally differentiated cortical fields are believed to constitute distinct functional or computational units, receiving particular types of input, executing a certain range of computations and directing their output to specific locations within extended networks. Thus, in order to study OMPFC connectivity in MDD one would need to know the number and position of the various cortical fields within this region.

Although cytoarchitectonic studies measure the anatomical properties of the cortex directly and thus provide "hard" scientific evidence, there is surprisingly little overlap between cytoarchitectonic parcellation maps from different studies. This is related to the fact that most studies are based on very few samples, thus not considering the inter-individual variability in cortical morphology and folding, the lack of quantitative criteria for the characterization of the fields in many of them and other methodological differences. MRI functional connectivity, in combination with graph theory metrics and clustering techniques, offers now the opportunity to approximate the results of cytoarchitectonic studies in delineating cortical computational units in vivo and probing their function(s) directly. In this way we can test the current hypotheses about cortical organization, replicate, refute and integrate 
them with other pieces of information about the cortex (such as receptor and myelo-architecture) that thus far we have been unable to incorporate in a comprehensive model of the human brain.

\section{Outline of thesis}

The aims of the current thesis were threefold: a) validate and extend data-driven methods based on MRI functional connectivity and graph theory to parcellate the cortex, b) apply these methods in the study of functional connectivity markers of MDD vulnerability and disease and c) identify trait biomarkers of BOLD changes during face processing in MDD. To achieve these aims we conducted a large empirical study with a sample of MDD patients, a sample of individuals with familial vulnerability to MDD and a sample of healthy controls. In total 120 subjects participated in one session of MRI scanning and behavioral testing.

The aim of the study described in Chapter 2 relates to our first goal. In this study we extended parcellation methods based on connectivity and graph theory to the OMPFC and delineated 19 functional clusters in a subset of the healthy controls. In addition, we tested the reliability and reproducibility of the method across hemispheres and runs and examined the extent to which our approach is robust in light of MRI-related artefacts in the ventralorbital part of the cortex. Finally, we used agglomerative hierarchical clustering to examine the large-scale organization within the OMPFC.

In the study of Chapter 3 we used the parcellation map we developed in our first study to systematically examine functional connectivity alterations within the OMPFC in MDD. Combining our data from the three groups of participants we sought to dissociate connectivity aberrations common to MDD vulnerability and disease groups, reflecting markers of MDD vulnerability and alterations unique to MDD patients, thus correlates of the disease state.

Finally, in our study of Chapter 4 we sought to determine which of the various abnormalities in BOLD activation during face processing previously reported for MDD acute phase also characterize the vulnerability state.

Chapter 5 summarizes the main conclusions drawn in the context of this thesis and discusses the implications of our findings for parcellation approaches and neuroscience applications in psychiatry. The discussion is extended by considering open issues and future directions. 


\section{References}

American, Psychiatric Association. 1994. Diagnostic and Statistical Manual of Mental Disorders, 4th ed. Washington DC: American Psychiatric Press.

Beck AT. 2008. The evolution of the cognitive model of depression and its neurobiological correlates. Am J Psychiatry 165: 969-77.

Biswal B, Hudetz AG, Yetkin FZ, Haughton VM, Hyde JS.1997. Hypercapnia reversibly suppresses low-frequency fluctuations in the human motor cortex during rest using echo-planar MRI. J Cereb Blood Flow Metab 17: 301-308.

Carvalho AF, Cavalcante JL, Castelo MS. et al. 2007. Augmentation strategies for treatmentresistant depression: a literature review. J Clin Pharm Ther. 32(5):415-28.

Ferrari AJ, Somerville AJ, Baxter AJ, Norman R, Patten SB, Vos T, Whiteford HA. (2012). Global variation in the prevalence and incidence of major depressive disorder: a systematic review of the epidemiological literature. Psychol Med. 1-11.

Fox MD, Buckner RL, White MP, Greicius MD, Pascual-Leone A. 2012. Efficacy of transcranial magnetic stimulation targets for depression is related to intrinsic functional connectivity with the subgenual cingulate. Biol Psych. 72(7): 595-603.

Fransson P. 2005. Spontaneous low-frequency BOLD signal fluctuations: an fMRI investigation of the resting-state default mode of brain function hypothesis. Hum Brain Mapp. 26:15-29.

Grotegerd D, Stuhrmann A, Kugel H, Schmidt S, Redlich R, Zwanzger P, et al. (2014). Amygdala excitability to subliminally presented emotional faces distinguishes unipolar and bipolar depression: An fMRI and pattern classification study. Hum Brain Mapp. 35:2995-3007.

Hayden EP, Hankin BL, Jordan PL. 2015. Parental Depression and Child Cognitive Vulnerability Predict Children's Cortisol Reactivity. Dev Psychopathol. 26:1445-1460.

Holma IAK, Melartin TK, Sokero TP. 2010. Major Depressive Disorder : A Five-Year Prospective Study. Am J Psych. 167:801-808.

Holtzheimer PE, Mayberg HS. 2011. Stuck in a rut: rethinking depression and its treatment. Trends Neurosci. 34:1-9.

Huettel SA, Song AW, McCarthy G. 2004. Functional Magnetic Resonance Imaging, Sinauer Associates Inc, Publishers Sunderland, Massachusetts, USA.

Kessler, RC, et al. 2005. Prevalence, severity, and comorbidity of 12-month DSM-IV disorders in the National Comorbidity Survey Replication. Arch Gen Psychiatry. 62(6): 617-627.

Klein DN. 2010. Chronic Depression: Diagnosis and Classification. Curr Dir Psychol Sci. 19: 96-100.

Logothetis NK, Pauls J, Augath M, Trinath T, Oeltermann A. 2001. Neurophysiological investigation of the basis of the fMRI signal. Nature. 412:150-7. 
Lohoff FW. 2010. Overview of the genetics of major depressive disorder. Curr Psychiatry Rep. 12(6):539-546.

Mayberg H. 2003. Modulating dysfunctional limbic-cortical circuits in depression: towards development of brain-based algorithms for diagnosis and. Br Med Bull.193-207.

Mayberg HS, Lozano AM, Voon V, McNeely HE, Seminowicz D, Hamani C, et al. 2005. Deep brain stimulation for treatment-resistant depression. Neuron. 45: 651-60.

Mourão-Miranda J, Almeida JRC, Hassel S, de Oliveira L, Versace A, Marquand AF, et al. 2012. Pattern ecognition analyses of brain activation elicited by happy and neutral faces in unipolar and bipolar depression. Bipolar Disord. 14: 451-460.

Ongür D, Price JL. 2000. The organization of networks within the orbital and medial prefrontal cortex of rats, monkeys and humans. Cereb Cortex .10: 206-19.

Pezawas L, Meyer-Lindenberg A, Drabant EM, Verchinski BA, Munoz KE, Kolachana BS, et al. 2005. 5-HTTLPR polymorphism impacts human cingulate-amygdala interactions: a genetic susceptibility mechanism for depression. Nat Neurosci. 8: 828-34.

Pizzagalli DA. 2010. Frontocingulate Dysfunction in Depression: Toward Biomarkers of Treatment Response. Neuropsychopharmacology. 36:1-24.

Price JL, Drevets WC. 2010. Neurocircuitry of mood disorders. Neuropsychopharmacology 35 192-216.

Raichle ME, MacLeod AM, Snyder AZ, Powers WJ, Gusnard DA,Shulman GL. 2001. A default mode of brain function. Proc NatlAcad Scl. 98:676 -682.

Riva-Posse P, Holtzheimer PE, Garlow SJ, Mayberg HS. 2013. Practical considerations in the development and refinement of subcallosal cingulate white matter deep brain stimulation for treatment-resistant depression. World Neurosurg. 80(27):25-34.

Skundberg-Kletthagen H, Hedelin B, Wangensteen S. 2015. Burden, Health and Sense of Coherence among Relatives of Depressed Inpatients. Open J Nurs. 5:163-172.

Souery D, Papakostas GI, Trivedi MH. 2006. Treatment-resistant depression. J Clin Psych. 67:16-22.

Strimbu K, Tavel JA. 2011. What are biomarkers?. Curr Opin HIV AIDS. 5:463-466.

Stuhrmann A, Suslow T, Dannlowski U. 2011. Facial emotion processing in major depression: a systematic review of neuroimaging findings. Biol Mood Anxiety Disord. 1:10.

Sullivan PF, Neale MC, Ph D, Kendler KS. 2000. Genetic epidemiology of major depression: review and meta-analysis. Am J Psychiatry. 157:1552-62.

van Rijswijk E, van Hout $H$, van de Lisdonk E, Zitman F, van Weel C. 2009. Barriers in recognising, iagnosing and managing depressive and anxiety disorders as experienced by Family Physicians; a focus group study. BMC Fam Pract.10:52. 


\section{Chapter 1}

Weissman MM, Bland RC, Canino GJ, et al. 1996. Cross-national epidemiology of major depression and bipolar disorder. JAMA. 276(4):293-299. 


\section{Chapter 2}

Human orbitomedial prefrontal cortex: Intrinsic connectivity parcellation and functional organization

Zoe Samara, Elisabeth A.T. Evers, Alexandros Goulas, Harry B.M. Uylings, Grazyna Rajkowska, Johannes G. Ramaekers and Peter Stiers (submitted) 


\section{Abstract}

The orbitomedial prefrontal cortex (OMPFC) subserves functions such as decision making, reward and emotion processing. Human and monkey anatomical studies indicate the presence of various OMPFC cortical fields and suggest that these are further organized in two extended networks, a medial and an orbital one, with different functions. Despite recent advances in neuroimaging, it remains challenging to parcellate the OMPFC in vivo, mainly due to susceptibility artifacts. Here, we use MRI resting state functional connectivity (FC) and a data-driven modularity optimization algorithm to parcellate the OMPFC and examine whether the resulting subdivisions obey the dual organization framework. Results show that our parcellation delineates neuroanatomically plausible subdivisions which respect cytoarchitectonic trends and exhibit FC profiles similar to their spatial anatomical substrates. Hierarchical clustering shows that the whole brain FC profiles of the OMPFC subregions distinguish them in two groups, a medial and orbital one, which overlap with the organization proposed by Barbas and Pandya (1989) and Ongür and Price (2000). Finally, signal quality and reliability analyses demonstrate that results are robust, and replicate well across hemispheres and functional runs. The current parcellation scheme can be used as a framework for the examination of FC alterations within the OMPFC in disease states. 


\section{Introduction}

The orbitomedial prefrontal cortex (OMPFC) is an extended, structurally and functionally heterogeneous cortical region, instrumental for goal-directed decision making, reward representation and emotional processing (Kringelbach, 2005; Rushworth et al., 2007; Rolls and Grabenhorst, 2008; Rolls, 2014). The various OMPFC functions rely on complex interactions between the specialized neuroanatomical units that constitute the OMPFC. Cytoarchitectonic studies in monkeys and humans have revealed the existence of several anatomical subdivisions within the OMPFC (Brodmann, 1909; Carmichael and Price, 1994; Petrides and Pandya, 1994; Ongür et al., 2003; Mackey and Petrides, 2009; Uylings et al., 2010) and tracing studies in the macaque have identified unique patterns of connectivity for each of these cortical fields (Cavada et al., 2000; Yeterian et al., 2012). Moreover, an additional, large-scale organization of the OMPFC has been proposed in the rhesus monkey. Based on cytoarchitectonic data, Barbas and Pandya (1989) distinguished a mediodorsal and a basoventral trend, coursing through the medial and orbital surface of the PFC, respectively. Similarly, Carmichael and Price (1996) and Ongür and Price (2000) proposed, using data from tracer studies, a distinction between a "medial" and an "orbital" prefrontal network. Each of these networks consists of tightly interconnected subregions and is characterized by a distinct pattern of cortico-cortical connections with limbic, autonomic and other subcortical structures (Ongür and Price, 2000). Although valuable, the insights into cortical organization partly stem from animal research, and their extrapolation to humans involves many untested assumptions about the monkey-human homology. Moreover, due to the invasiveness of tracer techniques, direct human data observations of neural connectivity are lacking. Since human PFC function is critical for understanding not only healthy brain function but also several disease processes, in the last decade several research groups have focused on developing non-invasive, in vivo MRI techniques to parcellate the human brain into functional units and on quantifying the connectivity of the obtained parcels.

Numerous studies have demonstrated the feasibility of delineating neuroanatomically plausible cortical subdivisions within the human cortex in vivo using magnetic resonance imaging (MRI) anatomical or functional connectivity (FC) (e.g., Johansen-Berg et al., 2008; Beckmann et al., 2009; Kahnt et al., 2012; Sallet et al., 2013; Neubert et al., 2014; Yeo, et al., 2011; Kelly et al, 2012). The approach exploits the insight that functionally homogeneous cortical fields feature a unique pattern of anatomical connections (Krubitzer, 1995; Passingham, Stephan and Kötter, 2002), which provides their neurons with the required afferent input and sends their computational output to the appropriate locations. In line with this, Cohen et al. (2008) showed the existence of abrupt changes in FC profiles in a spatial array of cortical vertices. Independent of any prior information about a 
region's function or topography, these consistent edges matched in size and number known boundaries between cortical fields. Since then, a host of parcellation studies has been conducted. In these studies anatomical (diffusion-weighted probabilistic tractography) or functional (resting state lowfrequency BOLD fluctuations) MRI data are used to construct a matrix of connectivity for all voxels in a specified cortical region. Subsequently, the voxels are grouped into sub-regions or partitions based on the similarity of their connectivity profiles across the whole brain or with pre-specified target ROls. Aside from comparing the size and location on the cortical mantle of the delineated sub-regions, the parcellation maps obtained in the above mentioned studies are validated by comparing the connectivity profiles or fingerprints of each delineated field with the connectivity features of their (putative) homologues in the macaque monkey, either qualitatively (Kelly et al., 2010; Klein et al., 2010; Caspers et al., 2011; Margulies et al., 2009; Margulies and Petrides, 2013) or quantitatively using FC data from resting state scans in monkeys (Sallet et al., 2013; Neubert et al., 2014).

Cortical parcellation studies have delineated subdivisions and connectivity characteristics that accord with those described by invasive cytoarchitectonic and tracers methods, in the dorsal, ventral and lateral prefrontal cortex (Sallet et al., 2013; Neubert et al., 2014; Goulas et al., 2012), the parietal and temporal lobe (Barnes et al., 2012; Tomassini et al., 2007; Mars et al., 2011; Mars et al., 2012) and the insula (Deen et al., 2011; Kelly et al., 2012), and have even been applied to the entire cortex (Craddock et al., 2012; Yeo et al. 2012). Parts of the OMPFC have also been targeted. Using diffusion weighted imaging (DWI) connectivity, JohansenBerg et al. (2008) parcellated the subgenual part of the medial PFC, whereas Beckmann et al. (2009) partitioned the entire cingulate gyrus. Kahnt et al. (2012) used whole-brain resting state FC to delineate units within the orbitofrontal cortex. While these studies provide a first indication of the local anatomical and functional segregation within the human cingulate and the orbitofrontal cortex, they do not allow assessing the large-scale functional organization within the extended OMPFC region. To fill this gap, in the current study we employ a parcellation method to partition the entire OMPFC using resting-state $\mathrm{FC}$ with two aims: 1) obtain a fully data-driven partitioning of the OMPFC and investigate the validity of the fields obtained by comparing their spatial and connectional characteristics with current knowledge, 2) examine the organization of OMPFC fields in extended neural networks, as described in anatomical studies, based on their whole brain connectivity characteristics.

We use a graph theory based parcellation tool, the state-of-the-art Louvain modularity optimization algorithm (Meunier et al., 2009; Shen et al., 2010; De Meo et al., 2012), which enables the partitioning of the OMPFC at the individual level, thus avoiding loss of information or bias associated with group averaging. The parcellation is achieved in a fully data-driven, objective 
way without any assumptions about the number of subdivisions present or the degree of inter-subject consistency in the area to be parcellated (Cloutman and Ralph, 2012). This technique has been previously used to identify basal ganglia subdivisions (Barnes et al., 2010) and has been validated for the lateral PFC (Goulas et al., 2012). We demonstrate that the OMPFC cortical fields are delineated in agreement with established cytoarchitectonic parcellation schemes in humans and macaques and exhibit FC profiles similar to anatomical connectivity characteristics observed in monkeys. Moreover, the whole-brain connectivity profiles of the delineated fields divide them in two groups of regions, an orbital and a medial one, which resemble the proposed dual large-scale OMPFC organization. Finally, we show that our results are valid and reproducible across hemispheres and functional runs, despite the susceptibility-related signal loss in the OMPFC at 3T.

\section{Materials \& Methods}

\section{Participants + fMRI acquisition}

Thirty four psychiatrically and neurologically healthy subjects $(21$ females; mean age, 32.3 years; SD, 14.5 years) participated in one MRI scanning session after giving their informed consent. The scanning protocol included two resting state scans of approximately 6.5 minutes with identical scanning parameters and instructions. Participants were instructed to fixate on a cross at the center of the screen, keep their eyes open and refrain from intentionally engaging in specific mental tasks or falling asleep during the scan.

Scanning was conducted on a Siemens MAGNETOM Allegra 3T MRI head-only scanner. Head motion was constrained by the use of foam padding. For each subject, 153 T2*- weighted gradient echo planar images (EPI) with 41 slices were acquired (except for 6 participants for whom 203 images were available). EPI can suffer substantial loss of BOLD sensitivity and geometric distortions due to magnetic field inhomogeneities near air tissue interfaces. In order to minimize MRI signal loss and recover the true spatial signal positions in the OFC we: a) used an optimized echo time, b) tilted the slices $\left(\sim 30^{\circ}\right.$ angle), and c) generated a field map to offline correct susceptibility-related signal displacements. Imaging parameters for the resting state sequence were as follows: TR, $2500 \mathrm{~ms}$; TE, $25 \mathrm{~ms}$; flip angle, 90\%; matrix size, $128 \times 96$; and FOV, $256 \mathrm{~mm}$; distance factor, $20 \%$; resulting in a voxel size of $2 \times 2 \times 3 \mathrm{~mm}$. The gradient echo image used to generate the field map had the same grid and slice orientation as the functional images (TR, $704 \mathrm{~ms}$; TE, $5.11,7.57 \mathrm{~ms}$; flip angle, $60^{\circ}$ ). In order to enable the localization of functional data, a high-resolution T1-weighted image was acquired with the following parameters: TR, $2250 \mathrm{~ms}$; TE, $2.6 \mathrm{~ms}$; flip angle, 
90; FOV, 256mm; slice thickness, 1mm; matrix size, 256X256; number of slices, 192; voxel size, 1X1X1mm.

\section{fMRI preprocessing}

Preprocessing of fMRI data was performed using SPM 5 software (Welcome Trust Center for Neuroimaging, London, UK). The functional data were subjected to the following preprocessing: slice time correction, spatial correction using the field map, realignment, co-registration with the anatomical scan, normalization to the Montreal Neurological Institute (MNI) template, reslicing to $2 \mathrm{~mm}$ isotropic voxels and smoothing with a $6 \mathrm{~mm}$ full width half maximum (FWHM) Gaussian kernel. The T1-weighted images were segmented into grey matter, white matter and CSF tissue maps and these maps were later used in the analyses. Further, we removed nonneuronal contributions from the BOLD signal by regressing the following nuisance variables: the six realignment parameters obtained by rigid body head motion correction, the time series extracted from cerebrospinal fluid and white matter, the session specific mean and the intrinsic autocorrelations. The residual volumes of the multiple regression were Fourier band pass filtered $(0.01-0.1 \mathrm{~Hz})$.

Head motion has been shown to significantly distort measures of FC (underestimating long-range and overestimating short-range connectivity) while commonly used preprocessing steps (e.g. realignment of volumes to the first scan, regression of the realignment parameters) do not adequately counter its effect (Power et al., 2012; van Dijk et al., 2012). In order to reduce the bias of head motion in our data we took the following approach: 1) we estimated the scan-to-scan head motion and identified scans during which the frame-wise displacement exceeded a particular threshold (absolute motion difference in the $z$ direction $>0.4 \mathrm{~mm}(1 / 10$ of voxel size); rotation in the $x$ direction $>0.26^{\circ}$ (angle corresponding to $0.4 \mathrm{~mm} z$-displacement of frontopolar voxels, assuming the rotation point in the middle of the brain is $88 \mathrm{~mm}$ from the anterior end of the brain's frontal pole; Talairach and Tournoux, 1988)), 2) we marked and excluded the identified volumes together with the 1-back and the 2-forward frames (to avoid spin history assumptions' violations caused by movement; Power et al., 2012), and 3) we included in the analyses only participants for whom at least 120 volumes (i.e. 5 minutes; van Dijk et al., 2010) of resting data were available after the correction (mean duration, $6.4 \mathrm{~min}$; SD, $0.8 \mathrm{~min}$ ).

\section{OMPFC Intrinsic FC-based parcellation}

Our aim was to parcellate the entire OMFPC into distinct areas based on its resting state FC. FC at rest, reveals a type of "default" functional organization of the brain that while not confined by (as it reflects both direct and indirect connections) nevertheless strongly reflects the underlying structural wiring (e.g. Cloutman and Ralph, 2012; Miranda-Dominiquez et al., 
2014). Here we used the intrinsic FC of the OMPFC, i.e. the connectivity of all OMPFC voxels among themselves, to delineate distinct areas with the Louvain modularity-detection algorithm (Blondel et al., 2008). The graph theory metric of modularity is a statistic that quantifies the degree to which a network can be subdivided into groups of nodes with higher than chance connectivity in between them (Girvan and Newman, 2002). Applied in brain networks, it can be used to delineate neurobiologically meaningful functional units, called modules, which perform specialized neural processing (reflecting the balance between functional integration and segregation that characterizes brain organization) (e.g. Meunier et al., 2009; Goulas et al., 2012).

The first step in this procedure is the construction of a correlation matrix. After preprocessing and reslicing the data $(3 \times 3 \times 3 \mathrm{~mm}$ voxel size), we extracted from the first resting state scan of each participant the time-course of all OMPFC voxels in the left hemisphere using the intersection of the normalized grey matter mask of each individual with an OMPFC ROI mask (thus including only each subject's grey matter voxels). The ROI mask comprised of the following Automated Anatomical Labeling $(A A L)$ map labels: left superior orbital gyrus, left middle orbital gyrus, left inferior orbital gyrus, left medial orbital gyrus, left rectal gyrus and left anterior cingulate gyrus (WFU PickAtlas; Tzourio-Mazoyer et al., 2002; Maldjian et al., 2003; Maldjian et al., 2004). The mask also included the cortex region corresponding to the left frontal superior medial AAL label, extending dorsally until the horizontal border defined by the anterior cingulate AAL label (manually drawn using MRIcron; Rorden and Brett, 2000). We calculated the Pearson's $r$ correlation coefficient for every OMPFC voxel with every other OMPFC voxel Using the extracted timecourses and constructed an $\mathrm{N} X \mathrm{~N}$ correlation matrix.

A common step before the application of modularity is to binarize the correlation matrix by applying an absolute weight threshold (Rubinov and Sporns, 2010) such that weak or non-significant links, corresponding most likely to spurious connections, are discarded, while graph connectedness remains intact. Since the threshold value is arbitrarily determined and no sensible "rule-of-thumb" exists, we defined the following range of thresholds for the correlation matrix over which the parcellation algorithm was applied: $0.005,0.01,0.015,0.02,0.025,0.03,0.035,0.04,0.045$ and 0.05. From these thresholds we chose the one for which modularity was maximum while graph connectedness was not significantly affected.

To partition the binarized correlation matrix into distinct functional subunits we then employed the Louvain module detection algorithm (implemented in MatLab as part of the Brain Connectivity Toolbox; Rubinov and Sporns, 2010), one of the best performing algorithms (Lancichinetti and Fortunato, 2009) for fast and efficient detection of modules in extended networks. The Louvain algorithm operates on the maximization of modularity Q 


$$
Q=\sum_{i=1}^{k}\left[\frac{e_{i}}{m}-\left(\frac{d_{i}}{2 m}\right)^{2}\right]
$$

where ei is the amount of edges linking nodes within module $i$, di is the total amount of edges of module i nodes (i.e. degree of module $i$ ), and $m$ is the total number of edges in the graph (i.e. network degree). Large $Q$ values indicate the presence of community structure where the number of edges (i.e. functional connections) within modules is significantly higher than that expected by chance (Newman, 2006). Modularity maximization is a Nondeterministic Polynomial time problem (Fortunato 2010) and thus, the use of the Louvain algorithm requires multiple iterations. For each dataset we run 50 repetitions of the parcellation algorithm and at the end of this process selected the parcellation solution with the highest $Q$. The parcellation procedure results in the unique classification of every voxel in the OMPFC into one of the modules in the solution. This classification was transformed into a 3D parcellation module map in order to visualize the distinct modules identified in each subject. All the analyses were performed using MATLAB (Mathworks, Natick, MA, USA).

To test the significance of our parcellation results, we compared the obtained $Q$ value with the $Q$ value of one null model per individual dataset. Zalesky et al. (2012) have recently drawn attention to the fact that observations of brain networks that use correlation as a measure of connectivity are inherently more clustered than random networks. For this reason, they should be benchmarked against null networks that preserve the transitive structure of correlation networks. We created such null networks matched in terms of connection density and threshold to the original networks by randomizing the individual correlation matrices with the Hirschberger-QiSteuer algorithm (for algorithm and details see Zalesky et al., 2012), which based on Cholesky decomposition, generates randomly null covariance matrices with distributional properties matched to the observed matrices.

\section{Groupwise clustering}

After partitioning the OMPFC patch into modules in each individual dataset, we integrated the parcellation results at the group level based on the spatial similarity between the modules. Spatial similarity was defined as the inverse of the Euclidean distance between the center of mass (COM) of modules from two different parcellations. The integration progressed iteratively. At each iteration, the parcellation by parcellation cost matrix for matching every individual parcellation with every other individual parcellation was computed. Then, the pairs with the lowest matching cost were merged one after the other by weighted averaging of the COMs of assigned modules. The merged parcellations entered the next iteration level and the procedure was repeated until a final set of COMs was obtained. Matching of pairs of 
parcellations was accomplished by the modified Hungarian assignment algorithm (Munkres, 1957; Cao, 2008), and comprised of assigning modules from the two parcellations in a way that minimized the average distance (i.e., the cost) between the COMs of assigned modules.

Averaging of COMs from assigned modules during merging was performed in a weighted manner. Each module of each parcellation started at the first iteration with a weight of 1.0. Whenever, during the merging process, a module remained un-assigned (i.e., due to different numbers of modules in the two parcellations), it was penalized by decreasing its weight. As a consequence, the misfitting module's influence during averaging with another module at the next level was reduced. In the final set of COMs, modules with a weight $<0.5$ were eliminated. As a final COM-distance check, the $10 \%$ modules farthest away from the final group COM were eliminated from each cluster.

At the end of this procedure, each final COM represents a cluster of modules from individual parcellations merged into this common COM. Note that not every initial module is represented in every final cluster, due to different numbers of modules per parcellation and due to the final COMdistance check. Finally, for each cluster a new COM was computed as the average coordinates of all voxels with a positive within-module degree $z-$ score (Guimerá and Amaral, 2005) (i.e. only voxels/nodes tightly connected within their module had an influence on the clusters' COMs).

\section{Replicability of intrinsic FC parcellation}

To confirm the robustness of our parcellation results, we examined their replicability across hemispheres and across functional runs. To test the replicability across hemispheres we applied the connectivity-based parcellation and groupwise clustering described above in the right OMPFC of the same resting state run. To test the replicability across runs we repeated the parcellation for the left OMPFC of the second resting state run in the scanning session. We compared the similarity of the resulting groupwise cluster solutions between each hemisphere and run by plotting their spatial extent and visually inspecting their correspondence with our main results. In addition, we quantified the amount of overlap between the subject-level parcellation maps in the left OMPFC of the first and second functional run by calculating their normalized mutual information.

\section{OFC Signal coverage and inter-run correlation stability}

EPI suffers substantial loss of BOLD sensitivity near air tissue interfaces and FC metrics are sensitive to the levels of signal amplitude and signal-to-noise ratio (Golestani and Goodyear, 2011). To ensure that despite the presence of susceptibility artifacts in the OFC there was sufficient signal coverage for our FC analyses, we: 1) quantified the severity of signal dropout 
and 2) assessed the extent to which signal dropout affected the measures of connectivity.

To quantify the severity of signal dropout in the artifact-susceptible areas we calculated the relative signal intensity. After part of the preprocessing (images were slice-time corrected, realigned, unwrapped and normalized), we calculated the relative signal intensity of grey matter as the signal intensity of each voxel (averaged across time) relative to the mean signal intensity of all grey-matter voxels (Smits et al., 2007).

To assess the impact of signal dropout on the connectivity metric used, first we computed as a "general" measure of BOLD sensitivity, the signal-to-noise ratio of the time series (tSNR), then we estimated the stability of the whole-brain FC profiles of each OMPFC voxel across the two separate resting-state runs and finally we calculated the impact of tSNR on stability. tSNR was defined as the mean intensity of every voxel in the time-series divided by its standard deviation across time (Triantafyllou et al., 2011; Golestani and Goodyear, 2011). Inter-run stability of FC was calculated as the eta ${ }^{2}$ coefficient of every OMPFC voxel pair, equal to:

$$
e t a^{2}=1-\frac{S S_{\text {Within }}}{S S_{\text {Total }}}=1-\frac{\sum_{i=1}^{n}\left[\left(a_{i}-m_{i}\right)^{2}+\left(b_{i}-m_{i}\right)^{2}\right]}{\sum_{i=1}^{n}\left[\left(a_{i}-M\right)^{2}+\left(b_{i}-M\right)^{2}\right]}
$$

where ai and bi are the correlations of voxel $i$ in the first and second run respectively, $\mathrm{mi}$ is the mean correlation of both runs at position $\mathrm{i}$ and $\mathrm{M}$ is the grand mean of correlations across all locations in both runs. The eta ${ }^{2}$ coefficient varies from 0 (no similarity) to 1 (identical) and directly quantifies the difference in the values of the same voxel in the two runs (Cohen et al., 2008).

We tested whether the inter-run stability differed significantly between the individual clusters by running a general linear mixed model in SPSS using compound symmetry heterogeneous as covariance structure and cluster as a fixed effects factor. The estimation method was restricted maximum likelihood (maximum iterations $=150$ ). The advantage of general linear mixed models is that they allow the analysis of repeated-measures data in unbalanced designs, as is the case here with repeated but not complete observations of subjects' modules per groupwise cluster. To control the family wise error rate we used Holm's sequential rejective Bonferroni correction (Holm, 1979; Holland and Copenhaver, 1987). All pairwise comparisons among means were adjusted to a corrected alpha of 05 .

To test whether tSNR had a significant effect on the inter-run correlation stability we run the general linear model with the SPSS procedure Mixed again this time using tSNR as a covariate. The amount of variance explained by tSNR was calculated as: 


$$
r^{2}=1-\frac{\widehat{\sigma^{2}}}{\bar{\sigma}_{0}^{2}}
$$

where $r^{2}$ is the proportion of the variance explained, $\sigma$ is the standard error estimate in the model with tSNR as predictor and $\sigma 0$ is the standard error estimate of the null model.

\section{Cortical and subcortical FC profiles of OMPFC subdivisions}

To validate our parcellation solution and determine the degree to which the areas in our FC-based parcellation map correspond to cytoarchitectonic divisions and their anatomical connectivity, for every cluster we computed whole-brain FC profiles. The FC maps were created by placing spherical seeds of $4 \mathrm{~mm}$ radius at the COM of each subject's module and calculating Pearson's correlation coefficient between the seeds and the rest of the brain voxel-wise. This resulted in whole-brain $r$ maps for every module at the individual level. The maps were subsequently $r-$ to-Z transformed using Fisher's formula and grouped depending to the cluster they had been assigned to at the groupwise clustering step. Next, independent one-sample t-tests for every cluster were performed against the null hypothesis of absence of connectivity between the clusters and the rest of the brain. Multiple comparisons were cluster-level corrected (Chumbley and Friston, 2009) and the cluster extent necessary for the correction was determined with Monte Carlo simulations (implemented in Brain Voyager QX 2.8; Goebel et al., 2006). The voxel-level threshold of each map was set at $p=.01$, uncorrected, and the functional image matrix ( $79 \times 95 \times 69$ voxels) was modeled in an iterative procedure using the estimate of the map's spatial smoothness. Following 10.000 simulations, the minimum cluster-level threshold that yielded $p=.005$ was selected. We subsequently used CARET (http://brainvis.wustl.edu/wiki/index.php/Caret:About) to project the cluster-level thresholded FC maps of each cluster on a 3D cortical surface.

Since CARET is a surface only map, it is not possible to localize on its 3D brain models subcortical FC. Due to the instrumental role OMPFC plays in emotional processing, the setting of mood and reward representation (Drevets et al., 2008), we chose to separately illustrate the subcortical FC of the clusters in the form of spider plots. The FC of the OMPFC clusters with the following subcortical structures was plotted: the amygdala, the hippocampus, the hypothalamus, the thalamus, the thalamic mediodorsal and pulvinar nuclei, the caudate, the ventral striatum, the putamen and the periaqueductal gray. For the amygdala, hippocampus, thalamus, caudate and putamen we created bilateral ROI masks using the corresponding AAL labels. The masks for the ventral striatum and hypothalamus were created using the definition and instructions of Tziortzi et al (2011). The ventral striatum mask covers the area functionally defined as ventral striatum in connectivity studies and its dorsal boundary includes nucleus accumbens, 
medial caudate nucleus and rostroventral putamen (Tziortzi et al., 2011). For the thalamic mediodorsal nucleus and pulvinar, we placed spherical ROIs of $8 \mathrm{~mm}$ radius at $\mathrm{MNI}$ coordinates $\mathrm{x}=-6, \mathrm{y}=-18, \mathrm{z}=8$ and $\mathrm{x}=-15, \mathrm{y}=-33, \mathrm{z}=6$ respectively (Zou et al., 2009). The mask for the periaqueductal gray was manually drawn in MRIcron (Rorden and Brett, 2000).

The subcortical ROI masks were used, in combination with the cluster-level thresholded t maps, to extract the strength of the significant positive connections in each subcortical area. For each cluster, a vector was created that reflected the average Fisher's z connectivity strength (Taylor et al., 2009) between the specific cluster and each subcortical ROI. The average strength of each subcortical ROI with each cluster was normalized by dividing all values by the maximum average strength of each cluster. The resulting vectors were represented as spider plots that visualize the subcortical FC fingerprints of each cluster on a zero to one scale.

\section{Similarity of whole-brain FC profiles of the OMPFC subdivisions}

It has been shown in parcellation studies that while delineated fields exhibit unique patterns of connectivity, it is possible to detect groups of areas with highly similar connectivity profiles which often participate in the same extended networks (Cloutman and Ralph, 2012; Passingham et al., 2002). To examine whether our OMPFC subdivisions could be distinguished into spatially extended networks we used agglomerative hierarchical cluster analysis which does not involve a priori assumptions about the number of groups present in the data and outputs a "bottom-up" hierarchy of areas in the form of a dendrogram. First, we calculated the (dis)similarity matrix for the whole-brain connectivity profiles of all clusters using correlation as the similarity metric (1-r) and subsequently created a dendrogram to represent the hierarchies in the data. In order to select the most appropriate linkage method for the construction of the dendrogram, we run the analysis using the following linkage methods: centroid, average, single, median, complete, weighted and Ward. For each of these methods we computed the cophenetic correlation coefficient of the resulting dendrogram, a measure of how well the original distances in the data are represented. From these, we selected the linkage method that generated a dendrogram which contained clusters proceeding hierarchically (i.e. monotonic) and had the highest cophenetic coefficient.

\section{Results}

\section{OMPFC Intrinsic FC-based parcellation \& groupwise clustering}

We used the intrinsic FC of voxels within the OMPFC to parcellate individual cortical patches into modules with the Louvain module-detection algorithm. Although we performed the parcellation analysis in both hemispheres using separate patch masks and obtained comparable 
partitions, the results reported in this paper concern the left hemisphere, a choice guided by pragmatic considerations. Results for the right hemisphere are presented only in the section on the replicability of the parcellation analysis, below. The parcellation resulted in contiguous modules within the patch for all participants. The modularity values (Q-values) obtained at different density thresholds of a subset of the data $(\mathrm{N}=21)$ are depicted in Figure 1. The $Q$ values were high ( $>0.4)$ at all threshold levels, consistent with a modular data structure (Fortunato, 2010). In addition, the averaged across subjects observed $Q$ was significantly higher than the $Q$ of the null models $(t(33)=41.73, p<.000)$. These results are consistent with studies using similar methods (Meunier et al., 2009; Barnes et al., 2010; Goulas et al., 2012). While we favor the density threshold yielding the highest modularity, the threshold also negatively affects the connectedness of the thresholded graph, i.e., the proportion of nodes/voxels that are no longer connected with the rest of the graph. We report here on the modules obtained at the highest density threshold examined (i.e., 0.005), which while yielding the highest modularity (i.e. .08), still had high connectedness of the graph (i.e. 98.99\%), which was not significantly different from the graph connectedness at the previous threshold $(98.97 \%, \mathrm{t}(20)=1.27, \mathrm{p}=.22)$.

The number of modules found in different subjects ranged from 14 to 22 (mean, 17.4; SD, 1.8). Individual modules were grouped across participants into clusters in a recursive data driven clustering procedure, based on their Euclidean spatial proximity. The group-wise clustering revealed the existence of 19 group-representative clusters of modules in the left hemisphere, comprising minimally modules from 22 subjects $(65 \%$ of sample size). Each cluster was assigned a unique number in an arbitrary way. An overview of the clusters of modules, their COMs and their volume size in $\mathrm{mm} 3$ can be found in Table 1 and the group-wise cluster map is shown in Figure 1. Seven of the clusters, C01, C16, C02, C03, C15, C17 and $\mathrm{C} 18$ are located at the orbital surface of the hemisphere, clusters C06 and C04 occupy the ventrolateral PFC and clusters C07 and C08 are located on the insulo-opercular cortex. At the medial wall, clusters C05, C19, C12 and $\mathrm{C} 13$ are distributed across the cingulate, clusters $\mathrm{C} 10$ and $\mathrm{C} 14$ cover the dorsal part of the medial superior frontal gyrus and clusters C09 and C11 occupy the dorsal medial and lateral PFC. As mentioned above, not all subjects have a module that corresponds to one (or more) clusters (see discussion section for elaboration on this issue). Nonetheless, correspondence of the classified individual modules with the group clusters of the parcellation map is good as illustrated in Figure 1 for three representative subjects. 


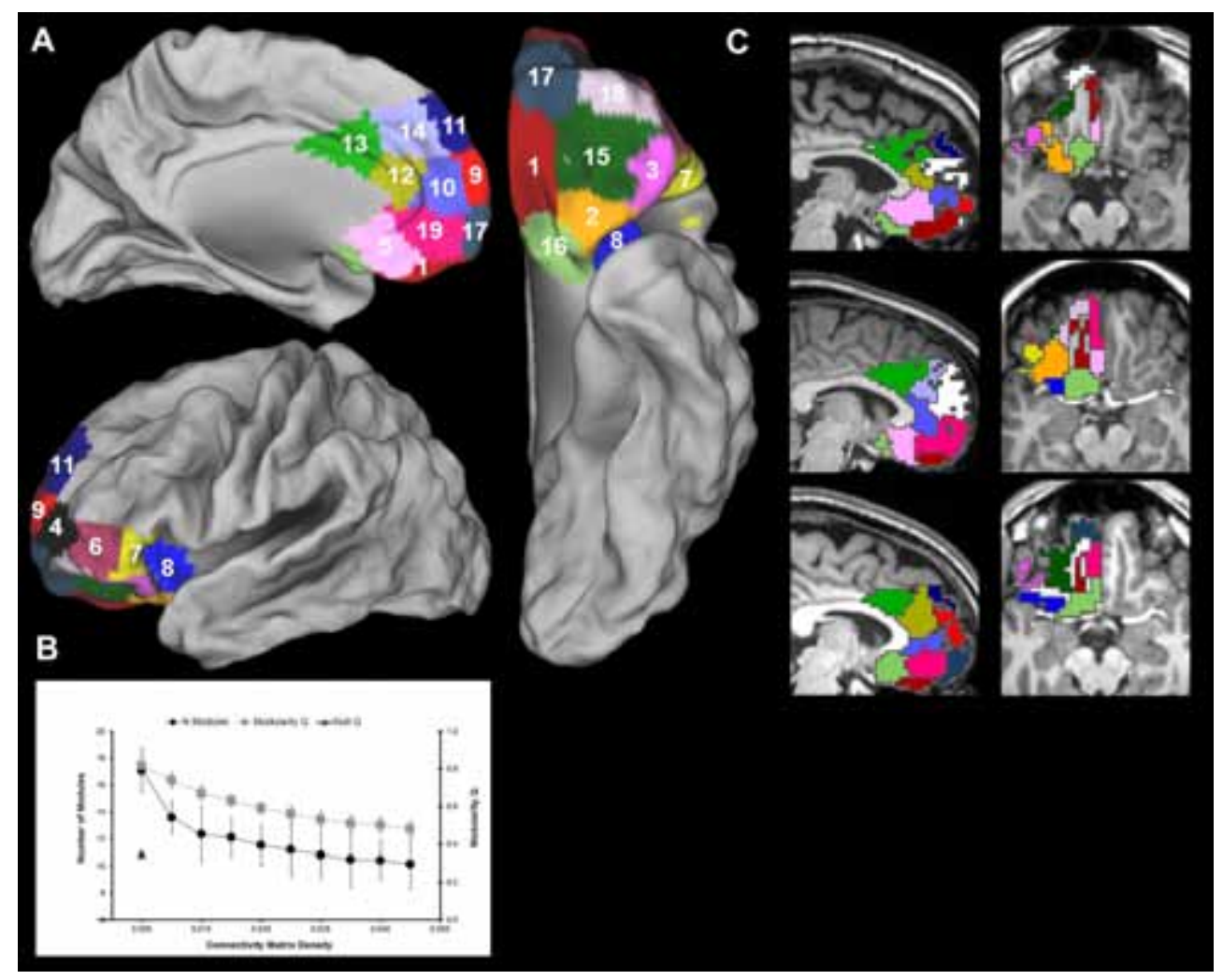

Figure 1. A. Groupwise parcellation map of clusters in the left hemisphere; each cluster is assigned a unique number and color. B. Number of modules for each connectivity matrix density threshold and modularity values for the real data at each threshold and the null model at .005. C. Parcellation maps of modules in three representative participants. Modules that correspond to the groupwise clusters plotted in 1.(A) haven been given the same color with the cluster they correspond to. Modules colored white were not assigned to any of the clusters. 
Table 1. Coordinates and size of the left hemisphere clusters.

Cluster numbers correspond to numbering of parcellation map (Figure 1). Reported coordinates are in MNI space and volume information concerns all modules corresponding to each cluster.

\begin{tabular}{|c|c|c|c|c|c|c|c|c|}
\hline \multirow[t]{2}{*}{ Cluster } & \multicolumn{5}{|c|}{ Center of Mass (COM) } & \multicolumn{2}{|c|}{ Volume $\left(\mathrm{mm}^{3}\right)$} & \\
\hline & \multicolumn{2}{|l|}{$\mathbf{x}$} & \multicolumn{2}{|c|}{$\mathbf{Y}$} & $\mathbf{Z}$ & & & \\
\hline & M & SD & M & SD & M & SD & M & SD \\
\hline $\mathrm{C} 01$ & -8.04 & 3.14 & 37.98 & 7.75 & -25.26 & 2.59 & $3,438.58$ & $1,282.34$ \\
\hline $\mathrm{C} 02$ & -26.38 & 3.24 & 23.99 & 5.64 & -21.22 & 2.46 & $3,330.72$ & $1,289.49$ \\
\hline $\mathrm{C03}$ & -35.81 & 6.30 & 33.61 & 4.39 & -15.52 & 2.16 & $2,917.13$ & $1,504.44$ \\
\hline $\mathrm{CO4}$ & -27.07 & 4.07 & 59.64 & 2.43 & -2.27 & 3.22 & $4,232.03$ & $1,352.18$ \\
\hline $\mathrm{C} 05$ & -2.79 & 0.70 & 29.27 & 5.91 & -14.09 & 3.00 & $4,317.30$ & $1,452.31$ \\
\hline $\mathrm{C} 06$ & -44.65 & 1.98 & 45.85 & 2.85 & -3.53 & 2.32 & $3,892.50$ & $1,406.99$ \\
\hline $\mathrm{C} 07$ & -46.29 & 3.03 & 28.67 & 3.88 & -5.48 & 3.47 & $4,130.13$ & $1,202.04$ \\
\hline $\mathrm{C08}$ & -35.20 & 3.36 & 20.49 & 1.74 & -9.02 & 2.66 & $4,860.00$ & $1,331.99$ \\
\hline $\mathrm{C} 09$ & -11.47 & 5.03 & 65.28 & 3.30 & 5.72 & 6.06 & $3,331.61$ & $1,424.13$ \\
\hline C10 & -5.00 & 1.38 & 50.97 & 5.13 & 2.18 & 5.85 & $4,313.48$ & $1,439.81$ \\
\hline C11 & -11.06 & 3.41 & 58.98 & 2.78 & 26.39 & 5.01 & $3,863.61$ & $1,291.57$ \\
\hline C12 & -4.48 & 1.69 & 41.51 & 4.73 & 12.03 & 4.15 & $3,877.00$ & $1,194.10$ \\
\hline C13 & -3.29 & 1.31 & 23.91 & 4.16 & 28.45 & 2.52 & $4,541.91$ & $1,363.20$ \\
\hline C14 & -5.06 & 1.23 & 43.10 & 6.27 & 27.71 & 4.04 & $4,172.46$ & $1,220.19$ \\
\hline C15 & -26.63 & 3.95 & 39.21 & 3.20 & -18.05 & 2.73 & $4,848.58$ & $1,771.68$ \\
\hline C16 & -14.54 & 4.62 & 15.11 & 2.79 & -19.45 & 2.30 & $4,475.25$ & $1,738.66$ \\
\hline C17 & -12.89 & 6.06 & 60.59 & 4.37 & -13.84 & 4.47 & $3,442.03$ & $1,448.43$ \\
\hline C18 & -30.25 & 7.15 & 53.54 & 4.71 & -13.33 & 4.27 & $2,911.09$ & $1,486.57$ \\
\hline C19 & -3.73 & 1.06 & 44.62 & 5.98 & -11.24 & 5.15 & $4,533.11$ & $1,427.86$ \\
\hline
\end{tabular}

Volume information concerns all modules corresponding to each cluster.

\section{Replicability of intrinsic FC parcellation}

To investigate the replicability of our results, the parcellation analysis was repeated in the left hemisphere of the second resting state run and the right hemisphere of the same functional run (data from the same participants). At the group level, the consistency of solutions across runs and hemispheres is depicted in Figure 2 where replicability results are overlaid on the main solution. Some shifts in boundaries can be observed, particularly in subregions having less pronounced cytoarchitectonic differentiation, such as 
in the orbital cortex and subdivisions of established cortical fields, as discussed below. Despite these local shifts, there is substantial overall agreement between parcellations at the group level.

At the individual level, parcellation modules obtained for the left hemisphere from the second run were quite similar as indicated by the high normalized mutual information between individual parcellations (mean, 0.7; $\mathrm{SD}, 0.04$ ). The correspondence of modules from different run and hemisphere in two representative participants is shown in Figure 2.

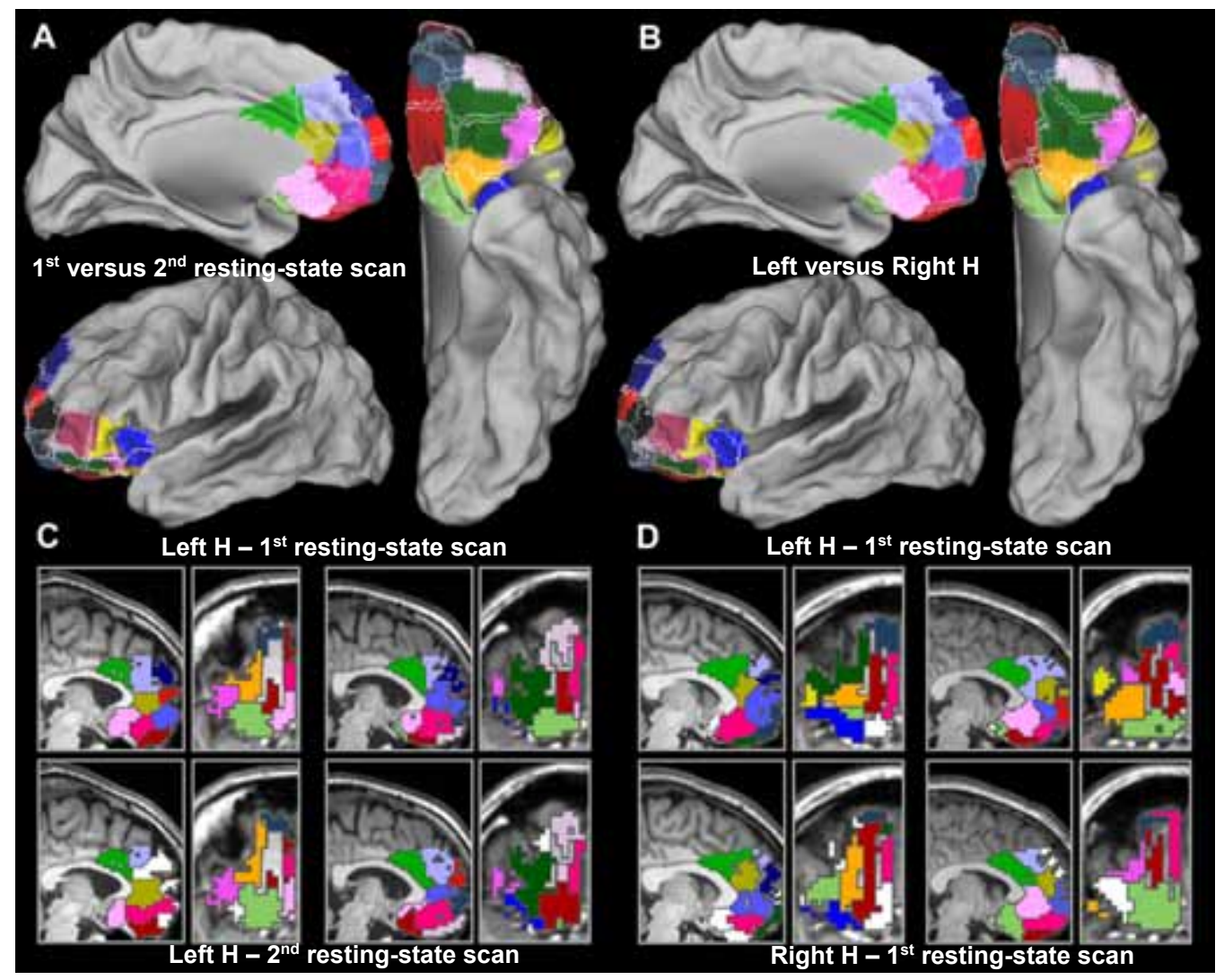

Figure 2. A. Replicability of parcellation map across functional runs. Clusters in the left hemisphere of the 1st resting state scan are plotted in color while the clusters in the left hemisphere of the 2 nd resting state scan are overlaid as borders in white. $\boldsymbol{B}$. Replicability of parcellation map across hemispheres. Clusters in the left hemisphere (1st resting state scan) are plotted in color while the clusters in the right hemisphere are overlaid as borders in white. C. Top panels (medial wall left, orbital surface right) depict parcellation module maps in the left hemisphere of the 1st resting state scan in two representative participants. Color coding corresponds to groupwise cluster maps. Bottom panels show the parcellation module maps of the same subjects in the left hemisphere of the $2 n d$ resting state scan. White modules were not assigned to 
any of the clusters. D. Top panels (medial wall left, orbital surface right) depict parcellation module maps in the left hemisphere of the 1st resting state scan in two representative participants. Color coding corresponds to groupwise cluster maps. Bottom panels show the parcellation module maps of the same subjects in the right hemisphere of the same scan. White modules were not assigned to any of the clusters.

\section{OFC Signal coverage and inter-run correlation stability}

The orbital part of the PFC is known to be susceptible to BOLD signal loss (Smits et al., 2007). This was also the case in our data set, despite the employment of an imaging sequence optimized for BOLD sensitivity in this area. As shown in Figure 3, relative signal intensity was decreased in the OFC, among other regions. The signal loss resulted in decreased temporal signal to noise ratio (tSNR) in this part of the brain (Figure 3). In addition to tSNR Figure 3 shows the inter-run FC stability for voxels in the parcellation patch averaged across participants. To get a better impression of the relationship between the signal quality and the parcellation results, we averaged the stability values per cluster of modules obtained in the parcellation analysis (cf. infra; Figure 3 ). The average eta ${ }^{2}$ ranged from .56 in orbital cluster $\mathrm{C} 16$ to .63 in medial cluster $\mathrm{C} 19$. For the clusters occupying the medial wall the average stability was .62 while the average eta ${ }^{2}$ of the orbitofrontal clusters was .59. While these values are close, there are nonetheless significant stability differences between orbital and medial clusters, as evidenced by the repeated-measures analysis (alpha 0.5; Holm's sequential rejective Bonferroni correction used to control the family wise error; Holm, 1979; Holland and Copenhaver, 1987). Orbital clusters C16, C01 and $\mathrm{C} 17$ had the lowest inter-run correlation stability, differing significantly from clusters both at the medial wall $(p=.000)$ and the lateral side $(p=.00)$. The rest of the orbital clusters $(C 18, C 15, C 02$, and $C 03)$ had a higher eta ${ }^{2}$ which differed significantly only from the medial wall clusters with the higher stability (Figure 3 ). The analysis with $\mathrm{tSNR}$ as a covariate revealed that $\mathrm{tSNR}$ had a small, albeit significant, $(F=30.1, p=.000)$ effect on the inter-run correlation stability, accounting for only $10 \%$ of its variance. Overall, our quality analysis indicates that the signal dropout inherent in EPI imaging does affect signal strength (tSNR) in the orbital surface compared to other cortical regions. However, its effect is restricted on the replicability of connectivity profiles of individual voxels over runs.

\section{Cortical and subcortical FC profiles of OMPFC subdivisions}

In order to validate our OMPFC partition we compared the relative position and extent and the FC profiles of our 19 clusters with various cytoarchitectonic maps (Figure 4) and macaque anatomical connectivity fingerprints. 


\section{Orbital Clusters}

On the orbital surface of the PFC we observed 7 clusters, C01, C02, C03, C15, C16, C17 and C18 (see Figure 5 for the cortical and subcortical FC profiles of each orbital cluster and Table 2 for an overview of all clusters' candidate anatomical substrates). In cytoarchitectonic studies of the human and macaque OFC, an anterior-posterior and a medial-lateral trend have been observed such that laminar differentiation of (mainly) layer IV and anatomical connectivity with distributed cortical and subcortical fields varies along these axes (Uylings et al., 2010; Cavada et al., 2000). These anteriorposterior and medial-lateral cytoarchitectonic and connectional trends are reflected in functional imaging studies (Kringelbach and Rolls, 2004; Kringelbach, 2005). In our results, along the anterior-posterior dimension, clusters $\mathrm{C} 01, \mathrm{C} 15$ and $\mathrm{C} 03$ are anterior, whereas $\mathrm{C} 16$ and $\mathrm{C} 02$ are posterior.

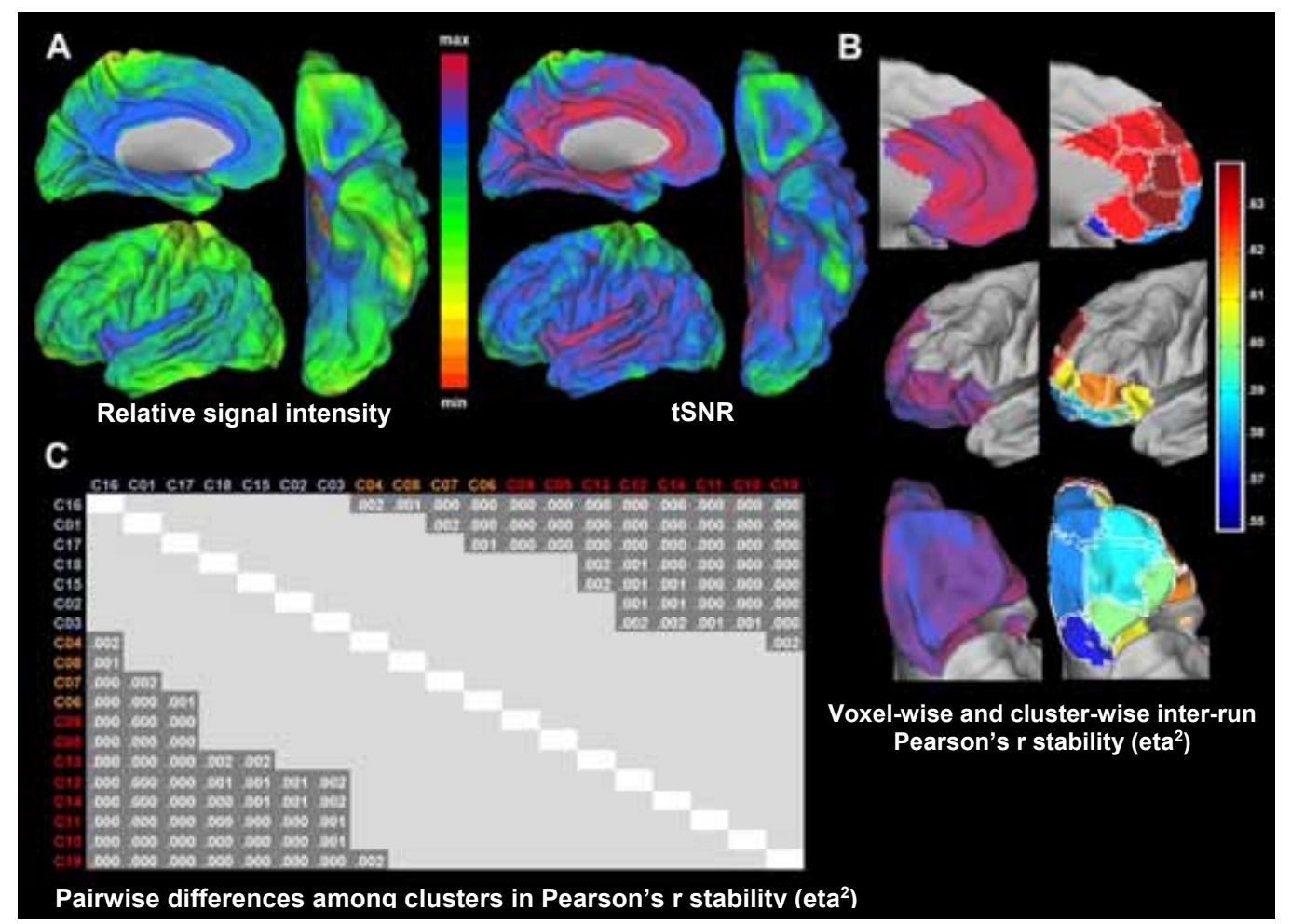

Figure 3. A. Whole brain relative signal intensity (left) and tSNR (right). Values range from 0 to 1.6 for signal intensity and 0 to 24.0 for tSNR. B. Left panel shows the stability of correlations (eta $\left.{ }^{2}\right)$ within the OMPFC voxel-wise. Values range from 0 to .7. Right panel shows the mean stability of correlations (eta $\left.{ }^{2}\right)$ for each cluster. $\mathbf{C}$. Significant pairwise differences of correlation stability $\left(\right.$ eta $\left.^{2}\right)$ among clusters ( $p$ values Bonferroni - Holm corrected). Orbital clusters are shown in blue, lateral and insuloopercular in orange and medial clusters in red. 
In the medial-lateral dimension, clusters C01, C15 and C03 spatially agree with the recent subdivision proposed by Uylings et al. (2010) into an area 11 , and areas $47 \mathrm{~m}$ and $47 \mathrm{l}$, with the olfactory sulcus and the lateral orbital sulcus grossly marking the boundaries between this three-way division. In the anterior-posterior direction, the spatial location of our division line is located somewhat more posterior than the division proposed by Uylings et al. (2010), and agrees more with a transition zone noted by Beck (1949). Many authors have noted, however, that the anterior-posterior division is not marked by sharp boundaries but rather constitutes a gradual cytoarchitectonic trend that includes a quite wide transitional zone (Uylings et al., 2010) as opposed to the medial-lateral division which is marked by sharper transitions. The FC profiles of our clusters vary along these dimensions, in agreement with macaque tracing studies (Cavada et al., 2000; Ongür and Price, 2000), as detailed below.

On the medial edge of the orbital side cluster C01 occupies all but the caudal part of the gyrus rectus and extends to the medial orbital gyrus. The COM of this cluster is located in the medial olfactory sulcus $(-8,38,-25)$. Cluster $\mathrm{C} 01$ has - in addition to coupling with the immediately adjacent ventral medial regions - confined connectivity with the posterior cingulate region (area 23d of Vogt et al., 2005) and the visual part of the precuneus (Margulies et al., 2009), and with the dorsal medial cortex (presumably BA 9), which was recently implicated in social cognition (Rushworth et al., 2013).
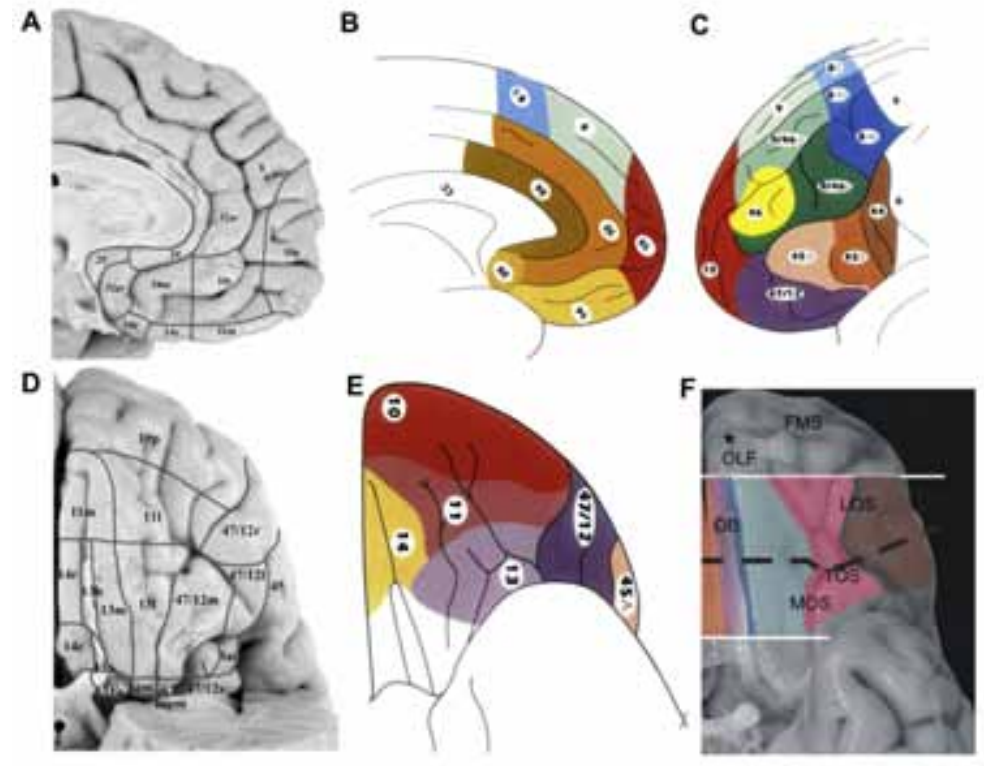

Figure 4. Cytoarchitectonic parcellations of the human medial $(A-B)$ and lateral $P F C$ (C) and the orbitofrontal cortex (D-F). A, D. Ongür et al., (2003). B, C, E. Petrides 
and Pandya (1994). F. Uylings et al., (2010). (Light blue=area 11; pink=area 47m; brown=area 47I)

Ventrally this cluster has focal coupling with the later orbital gyrus (our cluster C03). On the lateral side C01 is functionally connected with the ventral prefrontal cortex extending towards the anterior bank of the precentral sulcus, the angular gyrus and the lower aspect of the lateral temporal lobe. C01's pattern of FC largely overlaps with the anatomical connections seen in the presumed homologue macaque area 14 (reviewed in Yeterian et al., 2012; Morecraft et al., 1992; Carmichael and Price, 1996; Petrides and Pandya, 2012). The medial OFC is implicated in the value-weighting of choice options and the value-representation of chosen options (Rolls and Grabenhorst, 2008; Rushworth et al., 2011).

The cluster just lateral to $\mathrm{C} 01$ is cluster $\mathrm{C} 15$. It covers the anterior and part of the posterior orbital gyrus and laterally extends towards the medial orbital gyrus, with its $\operatorname{COM}(-27,39,-18)$ at the anterior orbital gyrus. In terms of functional coupling, C15 is in many respects the opposite of cluster $\mathrm{C} 01$. On the medial side it is connected with the dorsal aspect of the precuneus instead of the ventral limbic portion (Margulies et al., 2009), and with midcingulate motor areas (Amiez and Petrides, 2014) instead of the anterior cingulate gyrus. On the lateral side, C15's signal is significantly coupled with the anterior aspect of the supramarginal and the intraparietal instead of the angular gyrus, with the middle (presumed area 46; Goulas et al., 2012) instead of the inferior frontal gyrus, and with the posterior instead of the midsection of the lateral temporal lobe. Moreover, there is also coupling with the frontal eye fields and the dorsal-anterior (cognitive) subdivision of the insula (Kurth et al., 2010). This distributed pattern resembles frontoparietal and ventral attention network connectivity (Yeo et al., 2011) and has been associated with attention engaging processes during task execution (Duncan and Owen, 2000; Fox et al., 2006; Corbetta et al., 2008; Stiers et al., 2010). A spatially co-localized albeit smaller cluster identified in a previous parcellation study showed a similar pattern of $\mathrm{FC}$ with task-associated brain regions (cluster 3 in $\mathrm{k}=6$ solution of Kahnt et al., 2012). C15's profile of FC overlaps with the connectivity profile ascribed by tracing studies to macaque area 11 (Yeterian et al., 2012). 


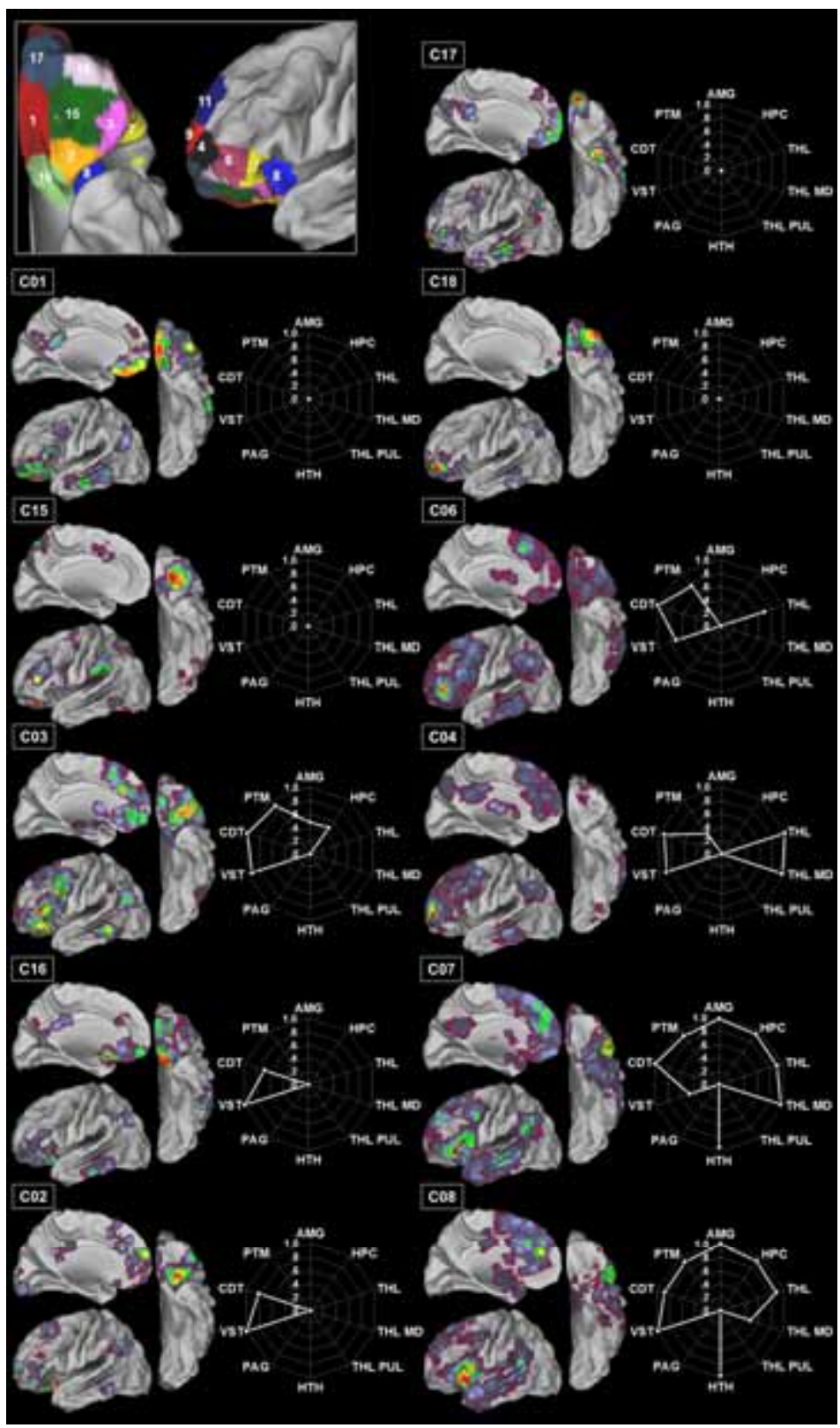

Figure 5. Whole-brain cortical and subcortical functional connectivity profiles of the orbital, ventrolateral PFC and insulo-opercular clusters. Bright colors in the cortical profiles represent higher $t$ values (voxel-level threshold $p=.01$; cluster-level threshold $p=.005)$. Radar plots illustrate separately the $F C$ of each cluster with the amygdala (AMG), hippocampus (HPC), thalamus (THL), mediodorsal (THL MD) and pulvinar (THL PUL) thalamic nuclei, hypothalamus (HTH), periaqueductal gray (PAG), ventral striatum (VST), caudate (CDT) and putamen (PTM). 
Our most lateral cluster on the orbital plane is cluster C03, covering the lateral orbital gyrus with its center at the mediocaudal part of the gyrus (COM -36, 34, -16). This spatial location corresponds to areas 47/12 (Petrides and Pandya, 1994; Mackey and Petrides, 2009; Ongür et al., 2003) and 4712 (Uylings et al., 2010). The FC of C03 differs from that of C15 and is again more similar to that of $\mathrm{C} 01$, but shows also distinctive features that parallel what is known for the connectivity of area 47/12 in the macaque (Yeterian et al., 2012). C03 has no posterior coupling in the medial wall, whereas its cingulate gyrus coupling is in between the subgenual focus of $\mathrm{C} 01$ and the mid-cingulate region of $\mathrm{C} 15$, with a strong focus in the anterior subpart of the mid-cingulate gyrus. On the lateral side, $\mathrm{C} 03$ shares with $\mathrm{C} 01$ the FC with the ventral aspect of PFC - albeit with a strong focus somewhat lower on the operculum - up to the posterior aspect of the middle frontal gyrus. However, $\mathrm{C} 03$ shows coupling with the dorsal aspect of the lateral $\mathrm{PFC}$, in contrast to $\mathrm{C} 01$ which lacks such connectivity. Further, we observe again that medial cluster $\mathrm{C} 01$ and lateral cluster $\mathrm{C} 03$ are functionally coupled. This, together with C03's strong coupling with all three major input nuclei of the basal ganglia, and with the amygdala and hippocampus, is consistent with the presumed role of the lateral OFC in learning and updating of the reinforcer and value association of choice options (Rushworth et al., 2011), information assumed to be communicated to the medial OFC (our C01) during decision making.

In the posterior OFC our medial-posterior cluster $\mathrm{C} 16$ occupies the caudal part of the gyrus rectus (COM -15, 15, -19) and is the spatial homologue of subregion 14c (Ongür et al., 2003; Mackey and Petrides, 2009). The FC pattern of $\mathrm{C} 16$ is similar to that of $\mathrm{C} 01$ on the posterior and ventral medial wall, but differs with respect to its dorsal anterior connectivity since it is functionally coupled with the dorsal cingulate cortex rather than the dorsal medial superior frontal gyrus as C01. Additionally, C16 shows FC with the anterior middle frontal gyrus rather than the operculum, parietal coupling extending in the IPS, inferior temporal rather than middle temporal connectivity and FC with the ventral anterior insula and the central orbital cortex (our C15).

Our lateral posterior cluster $\mathrm{C} 02$ in the caudal OFC covers the extent of the posterior orbital gyrus (COM -26, 24, -21). The FC profile of cluster $\mathrm{C} 02$ resembles that of lateral orbital cluster $\mathrm{C} 03$, but overall it is less strong. There are noteworthy differences, however, which agree with the tracer connectivity reported for macaque area 13 (Yeterian et al., 2012; Petrides and Pandya, 2012). While C02's anterior medial connectivity is similar to that of $\mathrm{C03}$, including the rostral mid-cingulate focus, $\mathrm{C} 02$ also shows posterior cingulate gyrus coupling. Moreover, C02's signal is significantly coupled with both the inferior temporal cortex of the ventral visual stream and lower visual cortical areas, (i.e. fovea representation). Conversely, C02 lacks the strong posterior lateral PFC connectivity that characterized $\mathrm{C} 03$. 
The anterior boundary between clusters C01-C15 and C17-C18 in our parcellation coincides with the boundary proposed by Uylings et al. (2010) for the division between the orbital cortex and the frontopolar cortex, comprising Brodmann's area 10 (Petrides and Pandya, 1994; Ongür et al., 2003; Mackey and Petrides, 2009). Human fMRI studies have implicated parts of BA10, in addition to lateral and medial orbital cortex, in reward-oriented decision making (Rolls and Grabenhorst, 2008). In line with this, the FC profiles of the fully differentiated cortical fields anterior to OFC appear as gradual extensions of the patterns observed in the OFC. Our medial cluster C17, located in the ventromedial part of the frontopolar surface and centered at the frontomarginal gyrus $(-13,61,-14)$, shares many connectivity characteristics with medial orbital cluster $\mathrm{C} 01$. The functional coupling pattern of $\mathrm{C} 17$ differs from that of $\mathrm{C} 01$ only in a few specific respects. On the orbital side $\mathrm{C} 17$ is functionally connected to the medial and not to the lateral side. In the lateral PFC, C17 showed no evidence of connectivity with the posterior operculum, but it does have functional coupling with the superior frontal gyrus (BA 9, our cluster C11). Lastly, C17 was the only cluster on the orbital plane for which we found temporal pole coupling. This confirms the reported DWI connectivity between an orbital division of area 10 and the temporal pole in humans (Liu et al., 2013). The temporal pole is considered paralimbic cortex and believed to be functionally implicated in the integration of emotional responses with highly processed sensory information and to be instrumental in the evaluation of emotional states (van Eijndhoven et al., 2013).

Adjacent to $\mathrm{C} 17$ in the anterior OFC lies cluster C18, immediately rostral to orbital cluster $\mathrm{C} 15$, on the lateral part of the frontopolar cortex, a cluster centered at the anterior orbital gyrus (COM -30, 54, -13).

Cytoarchitectonic parcellations do not agree on whether the cortical surface of the ventral frontal pole consists of one extended region or more divisions (Petrides and Pandya, 1994; Ongür et al., 2003; Drevets et al., 2008; Carmichael and Price, 1994; Hof et al., 1995). A recent cytoarchitectonic map showed that the human frontopolar cortex consists of one lateral frontopolar and one medial frontopolar division which are implicated in cognition, working memory and perception, and affective and social processing respectively (Bludau et al., 2014), while DWI imaging studies in humans (Catani et al., 2012; Liu et al., 2013; Moayedi et al., 2014; Neubert et al., 2014; but see Sallet et al., 2013 where one single presumed BA10 was identified) have also delineated more than one regions in the frontopolar cortex. Both our $\mathrm{C} 17$ and C18 are part of the lateral frontopolar subdivision Fp1 of Bludau et al. (2014) parcellation. Noteworthy is that in the only other FC parcellation of the OFC (Kahnt et al., 2012) the frontopolar-orbital surface of the left hemisphere was consistently divided in at least two clusters, across the range of $k=2$ to $k=7$ clustering solutions. In addition, a meta-analysis of PET and fMRI data has suggested functional subdivisions in frontopolar PFC (Gilbert et al., 2006). Although, statistical significance of FC for $\mathrm{C} 18$ was weak (mainly due to low 
inter-subject consistency), the features that could be discerned suggested that C18 is most likely a continuation of orbital cluster C15. C18 and C15 are the only clusters in this part of our parcellation patch that have clear functional coupling with the intraparietal sulcus, associated with the dorsal attention system (Fox et al., 2006; Corbetta et al., 2008). Additionally, they both have significant coupling with the posterior part of the lateral temporal lobe.

Orbital posterior clusters $\mathrm{C} 16$ and $\mathrm{C} 02$ together with the lateral $\mathrm{C} 03$ constitute the link of the OFC with subcortical structures in our parcellation. All three clusters showed strong connectivity with the striatum. While for the medial cluster $\mathrm{C} 16$ and central $\mathrm{C} 02$ this was confined to ventral striatum and caudate nucleus, $\mathrm{CO} 3$ showed equally strong coupling also with the motorrelated putamen, consistent with its strong affinity with the lateral PFC and the precentral gyrus. This pattern was also observed by Kahnt et al. (2012) who found that the medial part of posterior OFC had more FC with limbic striatum while the lateral part projected more to dorsolateral striatal area. Anatomical connections with the ventral striatum have been found in tracer studies for posterior regions of the macaque orbital cortex (Haber et al., 1995; Ferry et al., 2000), whereas the caudate nucleus has been found to receive projections from the lateral OFC (Selemon and Goldman-Rakic, 1985; Garrett et al., 1986; Haber et al., 1995). Putamen has been functionally linked with the lateral OFC, since inactivation of putamen in the macaque leads to selective impairment of reward history-based action selection (Muranishi et al., 2011). Moreover, individual variation in the monamine levels of monkey caudolateral OFC and the putamen interact to predict reversal learning performance (Groman et al., 2013). Functional coupling with the hippocampus was observed for our lateral cluster C03. FC of the lateral OFC with the hippocampus has been associated with learning of social cues (Ross et al., 2013), and is consistent with the role of lateral OFC in learning and updating stimulus-reward associations (Rushworth et al., 2011). In line with evidence in the macaque (Carmichael and Price, 1996; Amaral and Price, 1984; Ghashghaei et al., 2007) we found amygdala-OFC coupling for our posterior-lateral cluster $\mathrm{C03}$. Communication between the amygdala and the lateral OFC appears to be essential for updating of stimulus-reinforcer associations (Baxter et al., 2000; Rudebeck and Murray, 2011; Rushworth et al., 2011) and to be involved particularly in the processing of biologically salient stimuli such as food (Morris and Dolan, 2001). It should be noted here that tracing studies in macaques have found dense and highly efficient projections from the amygdala to posterior OFC (our cluster $\mathrm{C} 02$ ) that are believed to be critical for processing emotional content (Ghashghaei et al., 2007; Timbie and Barbas, 2014). However, we observed significant FC with the amygdala only for our lateral $\mathrm{C0} 3$ and not the posterior OFC C02. 
On the lateral boundary of our parcellation region several clusters of modules were found. Directly lateral of our lateral OFC cluster C03, we found cluster C06. C06 occupies the most anterior tip of the middle frontal gyrus (COM -45, 46, -4). C06's FC pattern resembles that of cluster C03. Similar to C03, C06 shows medial coupling with the ventral PFC and anterior paracingulate gyrus, and lateral coupling with dorsal and ventral lateral PFC. Conversely, unlike C03, C06 shares C18's coupling with the intraparietal sulcus and the middle inferior temporal lobe. Finally this cluster has widespread connections throughout the entire orbitofrontal plane. The extension of the FC pattern from lateral and anterior orbital surface on the ventrolateral PFC is consistent with the interpretation by Uylings et al. (2010) that the lateral OFC boundary should be drawn more laterally than most studies have recognized. Subcortically, $\mathrm{C} 06$ shares with $\mathrm{C} 03$ the significant coupling with the dorsolateral and ventral striatum, but communicates broadly with the thalamus instead of the hippocampus and amygdala.

Anterior to $\mathrm{C} 06$ and dorsal to $\mathrm{C} 18$, at the junction of the superior and middle frontal gyrus (COM -27, 60, -2) we delineated cluster C04. This cluster shares many FC features with $\mathrm{C06}$, but lacks its coupling with the OFC. Medially it shares C06's medial dorsal connectivity, but lacks its ventral coupling and has a stronger focus on the cingulate gyrus. Additionally, C04 shows posterior cingulate and precuneus connectivity, features which are absent from C06's map. On the lateral side, C04 is, similarly to C06, connected with the dorsal and ventral PFC, the middle inferior temporal gyrus and the intraparietal sulcus and the supramarginal gyrus. Finally, the subcortical connectivity of $\mathrm{C} 04$ parallels that of $\mathrm{C06}$, although with a more specific link to the mediodorsal thalamic nucleus.

Posterior to C06, on the ventral part of the frontal operculum, our parcellation includes cluster C07, with its $\operatorname{COM}(-46,29,-5)$ at the pars triangularis of the inferior frontal gyrus. C07 overlaps spatially with the ventral part of area 45A (Petrides and Pandya, 1994). Although our C07 includes only part of the cortex designated as area 45A, the pattern of its FC largely overlaps with the profile of area 45 seen in the macaque (Yeterian et al., 2012; Petrides and Pandya, 2012) and greatly resembles clusters delineated in previous human resting-state parcellations (Kelly et al., 2010; Ford et al., 2010; Goulas et al., 2012; Neubert et al., 2014; Margulies and Petrides, 2013). C07 is functionally connected to the dorsal and ventral medial PFC, to the posterior cingulate extending on the precuneus, has limited coupling with mainly the caudolateral OFC, and on the lateral side is functionally connected with the dorsal superior frontal gyrus, the temporal-parietal junction and the superior and middle temporal lobe. Subcortically $\mathrm{C} 07$ is strongly coupled with the amygdala, the hippocampus, the thalamus, the mediodorsal thalamus, the hypothalamus, the caudate, the putamen, and somewhat less strongly with the ventral striatum. 
Moving into the lateral sulcus, our parcellation yielded cluster C08, which spans the anterior part of the insular cortex and is centered at the lateral fissure $(-35,20,-9)$. Aside from strong intra-insular connections across the entire extent of the structure, C08 exhibited significant widespread connectivity with the dorsal medial cingulate and paracingulate cortex. Moreover, there is significant coupling with the entire extent of the superior and the middle temporal cortex and the temporal pole, the parietal and temporal opercula, the frontal operculum and the inferior frontal gyrus. This FC profile is consistent with the anatomical connections of the primate anterior insula (Augustine, 1996; Flynn et al., 1999; Mesulam and Mufson, 1982; Vogt and Pandya, 1987; Mufson and Mesulam, 1982). Similar connectivity has also been found in human resting-state connectivity and DWI studies (Cauda et al., 2011; Nelson et al., 2010; Deen et al., 2011; Cerliani et al., 2012). At the subcortical level, C08 is strongly coupled with the amygdala, hippocampus, thalamus, hypothalamus, ventral striatum, caudate and putamen, and somewhat less strongly with the mediodorsal thalamus. In the macaque, connections of the insula have been observed with these structures (Flynn et al., 1999; Augustine, 1996; Gallay et al., 2012; Chikama et al., 1997). Connections with the amygdala and the hippocampus have been confirmed also for humans in a recent DWI study (Cerliani et al., 2012). The anterior insular cortex links stimuli with emotional valence via intense, reciprocal amygdala connections and generally serves as a limbic integrative center for autonomic and sensory function (Devinsky and D'Esposito, 2004). The anterior insula/frontal operculum together with the dorsal ACC are considered core regions of a "salience network" responsive to stimuli relevant to emotional and/or motivational states and functionally linked with subcortical structures such as the extended amygdala, the mediodorsal thalamus and the hypothalamus, which are also involved in such responses (Medford and Critchley, 2010; Seeley et al., 2007).

\section{Medial Clusters}

On the medial wall of the PFC our parcellation yielded eight clusters of modules (see Figure 6 for the cortical and subcortical FC profiles of each medial cluster and Table 2 for an overview of the clusters' candidate anatomical substrates). Their overall spatial layout is in agreement with the organization described in the four-region neurobiological model of the cingulate cortex (Vogt et al., 2005; Figure 7E). According to this model, the prefrontal part of the cingulate gyrus is divided in a perigenual part, the anterior cingulate cortex (ACC), which includes cortical areas 25, and 24 and 32 proper, and a middle cingulate cortex (MCC) region comprising cortical areas 24' and 32' (Palomero-Gallagher et al., 2008). However, even within the ACC, rostrocaudal variation in cytoarchitectonic features is observed in both areas 24 and 32 (i.e., the cingulate and paracingulate gyri respectively; Koski and Paus, 2000; Yu et al., 2011; Margulies et al., 2007). The 
subdivisions of ACC's areas 24 and 32 are accompanied by functional differentiation (Vogt, 2005; Vogt et al., 2013; Palomero - Gallagher et al., 2013) a well-documented finding in human MRI studies (Yu et al., 2011).

In line with the above, in our parcellation we observed a chain of four clusters situated rostrocaudally around the corpus callosum (clusters C05, C19, C12 and C13). In the perigenual medial wall we found no segregation into more ventral and more dorsal clusters. Instead, our clusters $\mathrm{C} 05$ and C19 spatially covered the region of both area 24 and 32 . More posteriorly, this division was apparent, with clusters $\mathrm{C} 12$ and $\mathrm{C} 13$ covering mostly the cingulate gyrus, as does area 24/24' and our clusters C10 and C14 extending on the paracingulate cortex, similarly to area $32 / 32$ '. On the cingulate gyrus the boundary between the most posterior cluster $\mathrm{C} 13$ and the more anterior clusters $\mathrm{C} 12$ and $\mathrm{C} 14$ spatially corresponds to the distinction between the ACC and MCC regions of Vogt's model. More importantly, this discontinuity is also reflected in the FC profiles of these clusters. 


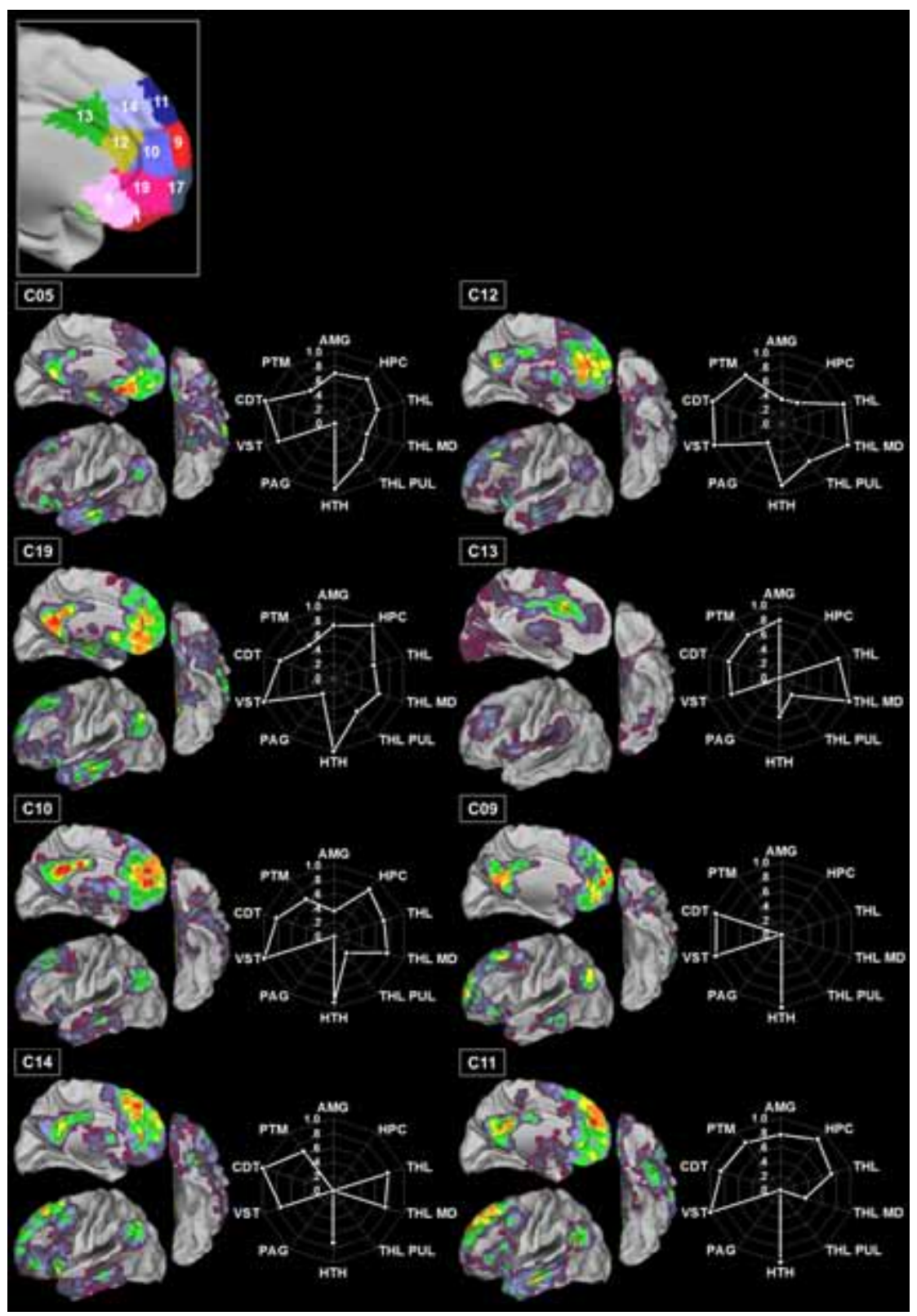

Figure 6. Whole-brain cortical and subcortical functional connectivity profiles of the medial clusters. Bright colors in the cortical profiles represent higher $t$ values (voxellevel threshold $p=.01$; cluster-level threshold $p=.005)$. Radar plots illustrate separately the FC of each cluster with the amygdala (AMG), hippocampus (HPC), thalamus (THL), mediodorsal (THL MD) and pulvinar (THL PUL) thalamic nuclei, hypothalamus (HTH), periaqueductal gray (PAG), ventral striatum (VST), caudate (CDT) and putamen (PTM). 
Table 2. Putative anatomical substrates for the left hemisphere clusters. Cluster numbers correspond to numbering of parcellation map (Figure 1). Candidate anatomical substrates are assigned for most clusters; see Figure 4 and cited papers for the cytoarchitectonic maps to which nomenclature refers to.

\section{Cluster Published Nomenclatures}

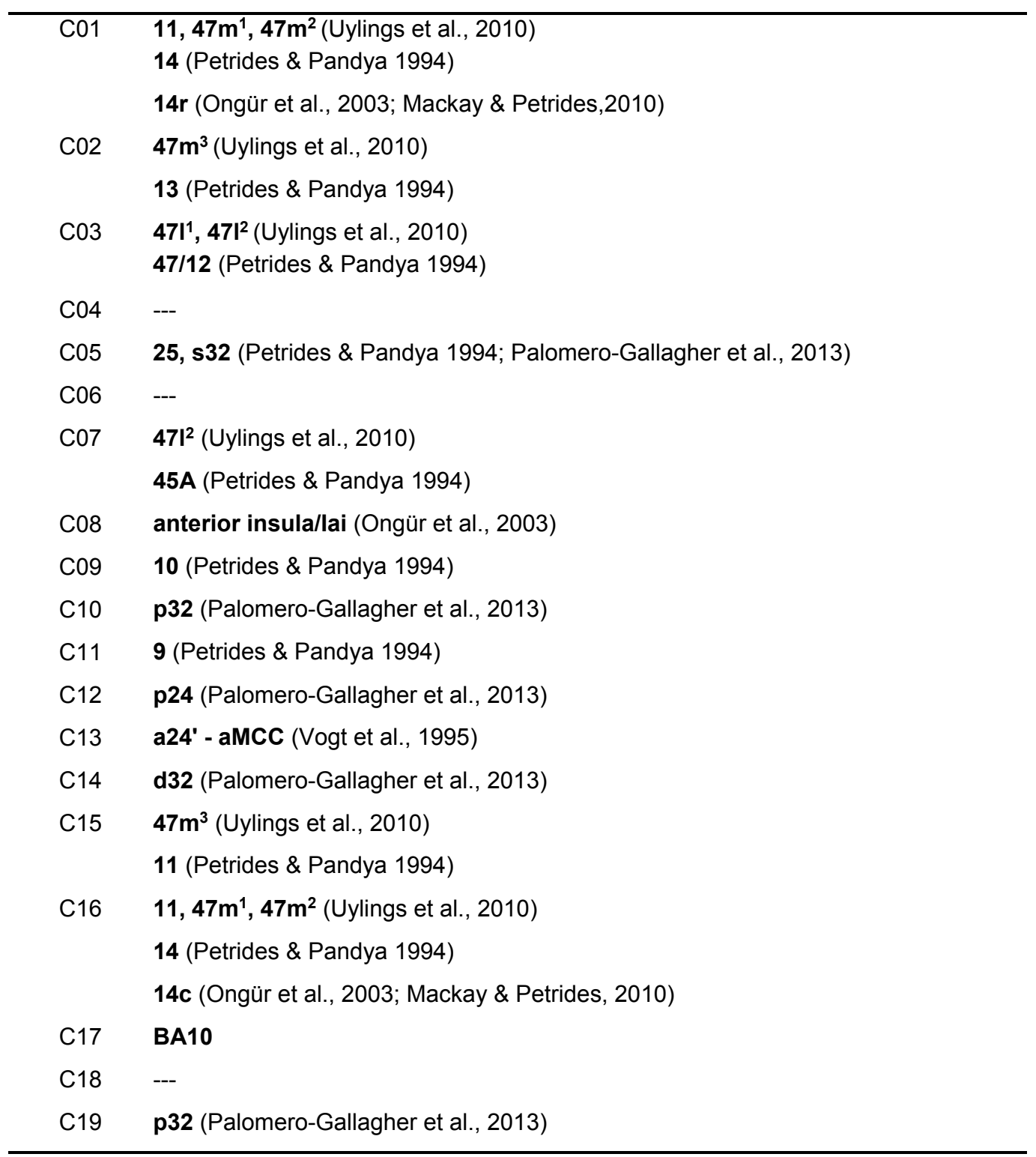


Although our ACC clusters constitute discrete subdivisions, they show rather gradual transitions in their cortical FC patterns (as opposed to the abrupt changes we observed at the orbital plane), with all clusters sharing a clear Default Mode Network (DMN) signature (Buckner et al., 2008). On the other hand, the pattern of subcortical connectivity that we observed for these clusters is more variable and distinct, with clusters having an overlapping yet distinct subcortical signature. For instance, a signature can be deciphered when taking into account the connectivity with the amygdala. Only C19 and C05 exhibit pronounced amygdala connectivity while the rest show modest levels of connectivity, as predicted by the amygdala- medial PFC connectivity of the macaque (Ghashghaei et al., 2007).

The most ventral cluster within ACC is cluster C05. It occupies the subgenual cingulate cortex and is centered $(-3,29,-14)$ at the paralimbic tier of the medial wall adjacent to the anterior subcallosal (paraolfactory) sulcus. Its spatial location and extent corresponds largely to cytoarchitectonic areas 25 and s32 (Petrides and Pandya, 1994; Palomero - Gallagher et al., 2013). On the medial side $\mathrm{C} 05$ is functionally connected to the posterior cingulate gyrus and the precuneus, the perigenual cingulate cortex and the medial superior frontal gyrus. On the lateral side $\mathrm{C} 05$ is significantly coupled with the superior and inferior frontal gyrus, the ventral anterior insula and temporal pole and the anterior-medial and lateral temporal lobe. Finally, on the orbital surface, $\mathrm{C} 05$ shows $\mathrm{FC}$ with the medial and lateral orbital cortex (our C01/C16 and C03). This FC profile corresponds well to the connectivity profiles ascribed to macaque areas 25 and 32 (Yeterian et al., 2012; Vogt and Pandya, 1987). Further, C05 shows connectivity with the angular gyrus extending towards the temporoparietal junction. This connectivity pattern is in keeping with human resting-state FC and DWI connectivity studies (Margulies et al., 2007; Yu et al., 2011; Johansen-Berg et al., 2008; Beckmann et al., 2009).

Dorsal to $\mathrm{C} 05$, along the paracingulate gyrus, we have found clusters C19, C10, and C14 which spatially correspond to subdivisions of area 32 (C19 and C10 to p32 and C14 to d32; Palomero-Gallagher et al., 2013). C19's COM $(-4,45,-11)$ is situated at the cortex above the 1st supraorbital sulcus, anterior of the corpus callosum genu, C10's COM $(-5,51,2)$ is located at the pregenual ACC, while C14 is centred $(-5,43,28)$ at the medial aspect of the superior frontal gyrus, dorsal to the cingulate sulcus. While these three clusters share many connectivity features with C05, they all have more pronounced FC with the medial parietal cortex (posterior cingulate gyrus and precuneus) and extensive connections with the dorsal and lateral PFC. Both these features are uniquely ascribed to area 32 in macaque tracing studies (Yeterian et al., 2012; Pandya et al., 1981). Similar coupling patterns have also been observed in a human resting-state FC study for seeds placed on the perigenual paracingulate cortex (seeds s5-7 in Margulies et al., 2007; see Cole et al., 2009 for issues of cross-species 
comparisons in ACC). While C19, C10 and C14, and to some extend also $\mathrm{C} 05$, are subdivisions of cytoarchitectonic area 32, with more similar cortical FC profiles, their subcortical connectivity differs. Clusters C05, C19 and C10 are all connected with the amygdala, the hippocampus, the thalamus (both the mediodorsal and the pulvinar thalamic nuclei), the hypothalamus and the ventral and dorsal striatum. However, only C19 is coupled with the periaqueductal gray. In addition, while coupling with amygdala is relatively strong for $\mathrm{C} 05$ and $\mathrm{C} 19$, it is only weak for $\mathrm{C} 10$. With respect to the striatum, all four clusters show coupling with its three major subdivisions, but for intermediate clusters $(C 19, C 10)$ it is relatively stronger with the ventral striatum, whereas for the clusters at the end points $(\mathrm{C} 05, \mathrm{C} 14)$ the coupling peaks in the caudate nucleus. The most caudal cluster $\mathrm{C} 14$ differs from the other three in having no connections with the amygdala, the hippocampus or the pulvinar thalamus.

On the perigenual cingulate gyrus, caudal to $\mathrm{C} 19$, we found cluster C12, with its COM located just anterior to the genu of the corpus callosum COM $(-4,42,12)$. This location corresponds to the caudal part of area 24 proper in the four region cingulate model (Palomero-Gallagher et al., 2008). In line with its spatial position, C12's FC profile corresponds to that of area 24 in the monkey (Vogt and Pandya, 1987; Pandya et al., 1981; Morecraft et al., 2012). This profile is characterized by prominent differences, compared to the connectivity of more rostral areas (our C05, C19) in the connection with the temporal pole (going dorsally on the cingulate, connectivity becomes more selective and then disappears), the OFC (C05 and C19 are connected with the medial and the caudolateral OFC while $\mathrm{C} 12$ is connected only to caudal OFC), the DLPFC (lateral connectivity extends progressively more posterior towards premotor areas and in the superior frontal sulcus), the cingulate ( $\mathrm{C} 12$ is the only ACC cluster that connects with the entire cingulate) and the frontal operculum (ventral cluster C05 connects mainly with the ventral operculum while connectivity for dorsal $\mathrm{C} 12$ extends towards the precentral sulcus). Subcortically, both caudal clusters C12 and C19 show FC with the periaqueductal gray, contrary to C05. Moreover, whereas C12 shares with the more rostral $\mathrm{C} 05$ and $\mathrm{C} 19$ connectivity with the ventral striatum and the caudate, it shows stronger coupling with the putamen. Contrary to C05 and C19, C12 has only weak coupling with the hypothalamus, but stronger coupling than these clusters with the mediodorsal thalamic nucleus. Lastly, it has weaker connectivity to the amygdala and the hippocampus compared to the other two clusters.

As already mentioned, Cluster C13, located caudally to $C 12$ on the cingulate gyrus, presented a discontinuity with the more rostral clusters $\mathrm{C} 12$ (area 24 proper) and C14 (32d) dorsally as well as the rest of the clusters on the medial wall. Both spatially and functionally, C13 seems to correspond to the most rostral motor cingulate region, mid-cingulate area a24' (Vogt et al., 1995). This is reflected in its FC profile, which is no longer characteristic of 
the DMN; rather, C13's FC on the medial wall is confined to the midcingulate region, while there is also strong coupling with the middle frontal gyrus laterally as well as the occipital cortex. C13 shows connections with areas from the task-positive network, the ventral attention and visual networks (Duncan and Owen, 2001; Fox et al., 2006; Corbetta et al., 2008; Stiers et al., 2010; Yeo et al., 2011) consistent with what is seen in the macaque for caudal 24-aMCC (Pandya et al., 1981; Vogt and Pandya, 1987). C13 is significantly coupled with the entire mid-cingulate cortex and preSMA, and on the lateral side with dorsolateral PFC (areas 9/46d, 46 and 44) and the dorsal anterior and middle insula. This pattern confirms previous findings in human resting state FC studies with seeds in the aMCC (Habas, 2010; Hoffstaedter et al., 2013) and is in line with the putative involvement of mid-cingulate cortex in motor preparation/implementation and error monitoring (Hoffstaedter et al., 2013). With respect to our subcortical ROls, C13 is strongly connected with the mediodorsal thalamus, and moderately with the pulvinar. Further, it is strongly coupled with the amygdala and the dorsal and ventral striatum and moderately with the hypothalamus.

Finally, on the dorsal boundary of our medial patch we have identified clusters C09 and C11 which appear to be the spatial and connectional homologues of medial frontal gyrus areas 10 and 9, respectively (Petrides and Pandya, 1994; Rajkowska and Goldman-Rakic, 1995). C09 occupies the anterior tip of the superior frontal gyrus (COM -11,65,6) and has a connectivity fingerprint characteristic of area 10 in tracing studies (Yeterian et al., 2012; Petrides and Pandya, 2012), including significant coupling with the medial parietal and the entire anterior medial frontal cortex. On the lateral side C09 has widespread connectivity with the superior frontal gyrus, the ventral inferior frontal and angular gyrus, and weak connections with the temporal pole and middle temporal gyrus. On the orbital side it is functionally coupled with medial, caudal and lateral orbital areas. The functional coupling of $\mathrm{C} 11$ (COM -11, 59, 26) differs from C09 in that on the medial side it has a focus on the anterior aspect of the posterior cingulate gyrus (area 23/p24' in Vogt's model) and on the pregenual ACC. On the lateral side, $\mathrm{C} 11$ shows less connectivity with the anterior middle frontal gyrus, and extensive connectivity with the anterior part of the temporal pole. On the orbital side we found evidence of $\mathrm{C} 11$ being coupled only with the orbital proisocortex. These characteristics have been described for area 9 in the macaque (Yeterian et al., 2012; Petrides and Pandya, 2012). Subcortically, C11 differs from C09 in that it has a profile strikingly similar to that of our insular/opercular clusters C08 and C07, being strongly connected to the hypothalamus, the ventral and dorsal striatum, the putamen, amygdala, hippocampus and the thalamus. On the other hand, C09 is also strongly connected to the hypothalamus but further has been found coupled only with the ventral striatum and the caudate. 


\section{Similarity of whole-brain FC profiles of the OMPFC subdivisions}

Aside from separately inspecting the FC profiles of our 19 OMPFC clusters, we wanted to examine whether the clusters formed extended networks, as proposed by Barbas and Pandya (1989) and Ongür and Price (2000) (see Figure 7C, and 7B, respectively, for the associated maps). Hierarchical agglomerative cluster analysis using correlation as the metric of similarity between the clusters' whole brain FC profiles and average linkage (cophenetic correlation coefficient $=.85$; cophenetic coefficient for other linkage methods were as follows: centroid $=.88$, single $=.84$, median $=.78$, complete $=.77$, weighted $=.77$, Ward $=.75$ ) resulted in the dendrogram of Figure 7D. At an intra-family distance of $65 \%$, the majority of OMPFC clusters are distinguished in two groups, a "medial" one which includes most clusters on the medial wall and an "orbital" one comprising of orbital and ventrolateral PFC clusters, while clusters $\mathrm{C} 13$ and $\mathrm{C} 15$ constitute singletons in the solution (see Figure 7A for a depiction of the spatial distribution of the groups).
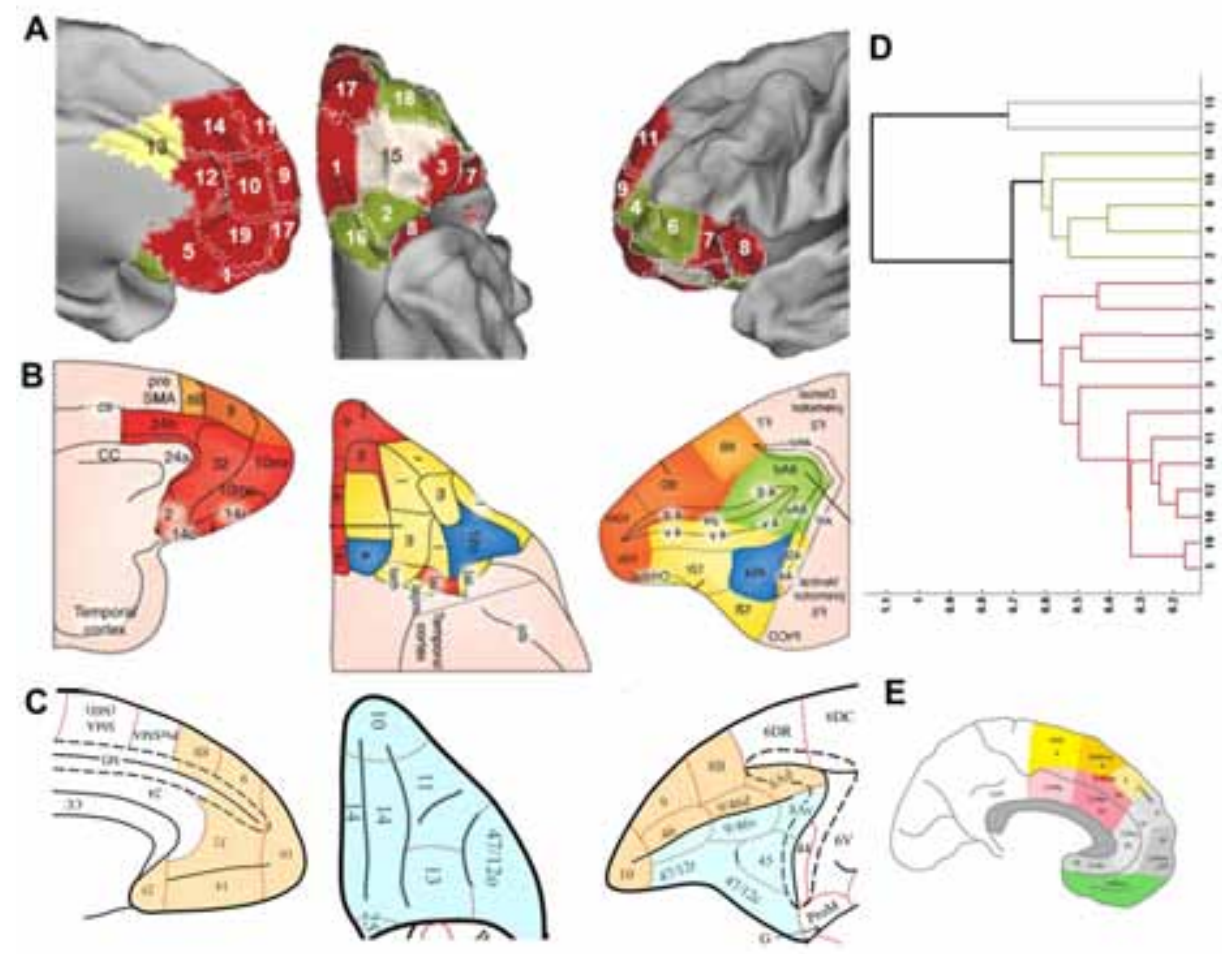

Figure 7. A. Cortical surface representation of the results of the hierarchical clustering analysis. OMPFC clusters belonging to the medial group are shown in red; clusters belonging to the orbital group are shown in green; singletons are uniquely colored. B. The medial (red) and orbital (yellow) networks proposed by Carmichael and Price (1996) and Ongür and Price (2000) (in Price and Drevets, 2010). Areas connected to more than one networks and believed to act as interfaces for 
information exchange are seen in blue. $\boldsymbol{C}$. The mediodorsal (orange) and basoventral (light blue) cytoarchitectonic trends described by Barbas and Pandya (1989) (in Yeterian et al., 2012). D. Dendrogram (average linkage) depicting the similarity of the whole-brain FC profiles of the OMPFC fields within each of the groups. E. Vogt's four-region neurobiological model of the cingulate (in Ullsperger et al., 2014). The border between ACC and MCC lies between areas shown in light grey and pink.

The medial group was formed by the anterior medial clusters ( $\mathrm{C} 05$, C19, C10, C12, C11, and C09 linked at an intra-family distance of $<.4$ ) and further included lateral OFC cluster C03, medial OFC clusters C01 and C17 and insulo-opercular clusters C08 and C07. As already discussed, the border between our most posterior cluster $\mathrm{C} 13$ and the more anterior clusters $\mathrm{C} 12$ and $\mathrm{C} 14$ marks the transition from ACC to MCC, a finding confirmed in the dendrogram with the $\mathrm{FC}$ profile of $\mathrm{C} 13$ being very dissimilar from all other medial wall clusters. Apart from $\mathrm{C} 13$, all medial wall clusters were grouped together, similar to the mediodorsal trend areas described by Barbas and Pandya (1989) in the macaque prefrontal cortex. The mediodorsal trend also includes areas 9 and 10 . Areas 9 and 10 have recently been recognized also by others as part of a dorsal prefrontal system (Price and Drevets, 2010; Saleem et al., 2014) which is tightly interconnected with the medial prefrontal network of Carmichael and Price (1996) and Ongür and Price (2000). In line with this, our medial clusters C09 and C11, which extend laterally into the dorsal PFC, were also grouped with the medial clusters. On the ventral side, our clusters $\mathrm{C} 01$ and $\mathrm{C} 17$, which cover the medial and rostral OFC, were also clustered in the medial group. This finding is also in accordance with the medial prefrontal network (Carmichael and Price, 1996; Ongür and Price, 2000), but disagrees with Barbas and Pandya's (1989) mediodorsal trend, which does not encompass the OFC.

As previously demonstrated (Yeo et al., 2011; Goulas et al., 2012), the families or groups created by hierarchical clustering are not constrained by spatial proximity and often reflect close functional synergy between remote areas. This appears to be the case for our insulo-opercular clusters $\mathrm{C} 08$ and $\mathrm{C} 07$ and lateral OFC cluster C03. Our finding that the ventral anterior insular cluster $\mathrm{C08}$ is connectionally more similar to clusters in the medial group fits with the designation of insular subdivision lai (insula agranular intermediate; Ongür et al., 2003) as part of the medial prefrontal network (Drevets et al., 2008; Saleem et al., 2014), as well as the repeated finding that the anterior insula is functionally linked to ACC (Medford and Critchley, 2010; Seeley et al., 2007). Finally, in our dendrogram, clusters C07 and $\mathrm{C} 03$ were also clustered with the medial group. Their presumed spatial anatomical substrates, areas $45 \mathrm{~A}$ and $47 / 12$ respectively have been found to have a "mixed" connectional profile, showing abundant anatomical connectivity with both the medial and the orbital prefrontal systems and are believed to subserve the exchange of information between the two (Price, 2007). 
The orbital group in our dendrogram was formed by the clusters of modules on the posterior and anterior central orbital surface $(\mathrm{C} 16, \mathrm{C} 02$, and C18). It also included clusters on the ventrolateral PFC (C04 and C06). This finding is partly in line with cytoarchitectonic findings. Barbas and Pandya's (1989) basoventral trend extends on the entire orbitofrontal plane, while Carmichael and Price's (1996) orbital prefrontal network involves mainly the cytoarchitectonic subregions of the central orbital surface and parts of the lateral OFC. In our orbital group, while posterior OFC clusters $\mathrm{C} 16$ and $\mathrm{C} 02$ and rostral cluster $\mathrm{C} 18$ are grouped together, our central OFC cluster $\mathrm{C} 15$ was found to have a very distinct whole brain FC profile and was, thus, not included in the group. On the other hand, the finding that ventral lateral PFC clusters C04 and C06 are connectionally similar to the rest of the areas in the orbital group agrees with the definition of the basoventral cytoarchitectonic trend which extends on the ventrolateral PFC until the principal sulcus. With regard to Carmichael and Price's scheme, while the related tracing studies initially included only the orbital surface, a recent similar analysis of the connections of the lateral PFC identified a ventrolateral prefrontal system, ventral to the principal sulcus, which is closely connected to the orbital prefrontal network (Price and Drevets, 2012).

\section{Discussion}

Various parts of the human OMPFC are instrumental for reward, affect and goal oriented behaviors and dysfunction in OMPFC fields underlies many severe psychiatric disorders. Our understanding of the OMPFC's role in complex behavior would benefit from the elucidation of its internal organization, which has been mostly studied with invasive techniques in primates. In the current study we used recently developed parcellation MRI analyses techniques to study the functional organization of this entire part of the cerebral cortex in vivo. Our approach was based on the intrinsic resting state FC of the OMPFC in combination with a modularity optimization algorithm. Our results show a consistent pattern of subdivisions in the OMFC across subjects, fMRI acquisition runs and hemispheres. Hence, they confirm previous studies demonstrating that resting-state $\mathrm{FC}$ can be used to parcellate the OFC (Kahnt et al., 2012) and that modularity-optimization leads to neuroanatomically plausible results (Barnes et al., 2010; Goulas et al., 2012). We have shown that the subregions we delineated agree with the cytoarchitectonic and connectional divisions reported in post-mortem studies of animal and human subjects.

Specifically, in the OFC, the discontinuous cytoarchitectonic change along the medial-lateral dimension and the more gradual transition from posteriorly to anteriorly (van Economo and Koskinas, 1925; Sarkissov et al., 1955; Ongür et al., 2003; Mackey and Petrides, 2010; Uylings et al., 2010) were mirrored in the fact that medial to lateral cluster boundaries were highly replicable across hemispheres and fMRI runs, whereas posterior-anterior 
divisions were more variable. These cytoarchitectonic trends were also reflected in the FC profiles of our seven OFC clusters. Functional variation along these dimensions was first recognized in a meta-analysis of activation studies by Kringelbach and Rolls (2004), who suggested that the complexity of reinforcers was coded gradually along the posterior-anterior dimension, while the medial OFC differed from the lateral in coding positive versus negative outcomes. Recently, a modified view emerged, in which medial OFC areas are thought to be concerned with values associated with internal processes while central OFC regions assign value to external stimuli (Rolls and Grabenhorst, 2008; Wallis, 2012). Alternatively, it has been suggested that medial OFC is responsible for comparing the values between options while lateral OFC is concerned with storing value-option associations (Rushworth et al., 2011). The fact that comparable transition trends are observed in FC-based parcellation results confirms the validity of these methods, and opens opportunities for future fMRI studies to investigate OFC activations against the background of independently delineated cortical divisions. However, questions still remain, such as the number of functional subdivisions of BA10, a phylogenetically young area that is believed to have evolved differently in humans compared to macaques (Bludau et al., 2014). In our study we replicate Kahnt's et al. (2012) finding of two subregions in the rostral/frontopolar PFC, with distinct FC profiles. However, this finding cannot be easily integrated with other studies on BA10 so far and thus, remains an issue to be clarified in the future.

Our intrinsic FC-based parcellation methods delineated neuroanatomically meaningful clusters also in the medial wall, where we replicated the spatial boundary between the ACC and MCC regions of Vogt's neurobiological model (Vogt et al., 2005) and the abrupt change in connectivity associated with it. Our findings in the medial PFC are in agreement with a previous resting state FC study showing that whole-brain FC profiles change from a typical DMN organization in the most anterior regions to a task-oriented attentional organization in the midcingulate regions (Margulies et al., 2007). Activation studies have revealed extensive regional specialization within the anterior medial cortex, which is implicated in the processing of conflict, reward, pain and emotion, but also suggest that subtle differences in task parameters (e.g. task complexity) modulate the recruitment of distinct ACC subregions (Torta et al., 2013). It remains unclear, however, how each of these functions and parameters map on the different ACC subregions (Etkin et al., 2011). Our results suggest that FC parcellation methods could help in clarifying the functional role of the different subregions. This is particularly the case for the medial wall cortex where, on the one hand, detailed anatomical connectivity profiles of each cytoarchitectonic field are far from complete and, on the other, correspondence between human and monkey homologues are still in dispute (Cole et al., 2009). 
Finally, we demonstrated that the whole-brain FC profiles of our OMPFC cortical fields are organized in a way similar to the basoventral and mediodorsal cytoarchitectonic trends described by Barbas and Pandya (1989) and the medial and orbital prefrontal systems of Carmichael and Price (1996) and Ongür and Price (2000) (see Figure 7A-C). These systems consist of areas that preferentially communicate internally through local cortico-cortical connections. Several studies have demonstrated that the medial and the orbital prefrontal systems have also distinct connections with the rest of the brain (An et al., 1998; Ongür et al., 1998; Ferry et al., 2000; Ongür and Price, 2000). The medial network comprises the anterior cingulate and superior medial frontal gyrus as well as the adjacent medial edge of the orbital cortex and a small region of the posterolateral orbital cortex. This network is involved in emotion processing and mood disorders, through its connections with other cortical and subcortical areas such as the anterior and medial temporal cortex, the posterior cingulate cortex, the amygdala, ventral striatum, the thalamus, the hypothalamus and the periaqueductal gray (Price and Drevets, 2012). The orbital network together with the ventrolateral prefrontal areas is primarily involved in the integration of multimodal sensory stimuli and the coding of the stimuli's affective value (Price and Drevets, 2012). This is reflected in their connections to several sensory as well as striatal areas. The two systems constitute a sensory-visceromotor link critical for the guidance of reward-related behavior and the setting of mood (Ongür and Price, 2000; Price and Drevets, 2012).

Despite the overall consistent results reported here, specific limitations of the present study need to be considered. While classical postmortem studies define cortical fields by marking potential field boundaries based on measurable discontinuities in anatomical features, the delineation of fields in in vivo imaging methods is dependent on the more gradually varying level of similarity in connectivity patterns of voxels. The critical amount of similarity for grouping voxels into modules is indirectly dependent on parameters of the algorithms, such as the number of centroids in clustering approaches, the number of components in linear decomposition methods, and the threshold used to binarize the correlation matrix in our approach. While this does not necessarily affect the quality and reliability of the parcellation results, it does pose a problem for interpreting and comparing results across studies. Cytoarchitectonic cortical fields may not coincide with functionally relevant subdivisions. For instance, a number of functionally meaningful subdivisions of BA6 have been proposed (Barbas and Pandya, 1987; Matelli and Luppino, 2001; Petrides and Pandya, 2006). Parameter settings resulting in fewer but larger modules might group cortical fields or subfields, whereas settings yielding smaller modules might divide fields into functional subunits. Moreover, optimal parameter settings might differ for different individual data sets and even different regions within one data set. This scaling problem - inherent in all current in vivo parcellation 
approaches -is reflected in our data in individual differences in parcellation between participants, while at the group level we obtain replicable results.

A second point of caution in the interpretation of our results is related to acquisition quality differences between the orbital and medial prefrontal cortex. While our quality metrics and results confirm that parcellation of the OFC is feasible, MR signal quality and connectivity stability over runs was somewhat lower in the medial and rostral OFC. This affects the quality of the FC maps for these regions. In the future, studies that take advantage of advanced MRI hardware options might further minimize the signal loss and yield more complete descriptions of the $\mathrm{FC}$ in these brain regions.

The above mentioned limitations notwithstanding, the results reported here warrant the conclusion that data-driven fMRI parcellation techniques succeed in delineating cortical fields in vivo that reflect what is known from anatomical studies about the OMPFC organization, both at the level of separate subregions and at the level of extended neural circuits. Thus, they open the way for systematically mapping structure-function relationships that underlie behavior, cognition and emotion in health and disease. 


\section{References}

Amaral DG, Price JL. 1984. Amygdalo-cortical projections in the monkey (Macaca fascicularis). J Comp Neurol. 230:465-496.

Amiez C, Petrides M. 2014. Neuroimaging evidence of the anatomo-functional organization of the human cingulate motor areas. Cereb Cortex. 24:563-578.

An X, Bandler R, Ongür D, Price JL. 1998. Prefrontal cortical projections to longitudinal columns in the midbrain periaqueductal gray in macaque monkeys. J Comp Neurol. 401:455-479.

Augustine JR. 1996. Circuitry and functional aspects of the insular lobe in primates including humans. Brain Res Rev. 22:229-244.

Barbas H, Pandya DN. 1987. Architecture and frontal cortical connections of the premotor cortex (area 6) in the rhesus monkey. J Comp Neurol. 256:211-228.

Barbas H, Pandya DN.1989. Architecture and intrinsic connections of the prefrontal cortex in the rhesus monkey. J Comp Neurol. 286:353-375.

Barnes KA, Cohen AL, Power JD, Nelson SM, Dosenbach YBL, Miezin FM, Petersen SE, Schlaggar BL. 2010. Identifying Basal Ganglia divisions in individuals using restingstate functional connectivity MRI. Front Syst Neurosci. 4:18.

Barnes KA, Nelson SM, Cohen AL, Power JD, Coalson RS, Miezin FM, Vogel AC, Dubis JW, Church JA., Petersen SE, Schlaggar BL. 2012. Parcellation in left lateral parietal cortex is similar in adults and children. Cereb Cortex. 22:1148-1158.

Beck E. 1949. A cytoarchitectural investigation into the boundaries of cortical areas 13 and 14 in the human brain. J Anat. 83:147-157.

Beckmann M, Johansen-Berg H, Rushworth MFS. 2009. Connectivity-based parcellation of human cingulate cortex and its relation to functional specialization. J Neurosci. 29:1175-1190.

Blondel VD, Guillaume JL, Lefebvre E (2008) Fast unfolding of communities in large networks. J Stat Mech P10008.

Bludau S, Eickhoff SB, Mohlberg H, Caspers S, Laird a R, Fox PT, Schleicher A, Zilles K, Amunts K. 2014. Cytoarchitecture, probability maps and functions of the human frontal pole. Neuroimage. 93: 260- 275.

Brodmann K. 1909. Vergleichende Lokalisationslehre der Grosshirnrinde in ihren Prinzipien Dargestellt auf Grund des Zellenbaues. Barth: Leipzig.

Buckner RL, Andrews-Hanna JR, Schacter DL. 2008. The brain's default network: anatomy, function, and relevance to disease. Ann N Y Acad Sci. 1124:1-38.

Cao Y. 2008. Hungarian algorithm for linear assignment problems V2.2. Available from: http://www.mathworks.com/matlabcentral/fileexchange/20652. Accessed 10 July, 2011.

Carmichael ST, Price JL. 1994. Architectonic subdivision of the orbital and medial prefrontal cortex in the macaque monkey. J Comp Neurol. 346:366-402. 
Carmichael ST, Price JL. 1996. Connectional networks within the orbital and medial prefrontal cortex of macaque monkeys. J Comp Neurol. 371:179-207.

Caspers S, Eickhoff SB, Rick T, von Kapri A, Kuhlen T, Huang R, Shah NJ, Zilles K. 2011. Probabilistic fibre tract analysis of cytoarchitectonically defined human inferior parietal lobule areas reveals similarities to macaques. Neuroimage. 58:362-380.

Catani M, Dell'acqua F, Vergani F, Malik F, Hodge H, Roy P, Valabregue R, Thiebaut de Schotten M. 2012. Short frontal lobe connections of the human brain. Cortex. 48:273291.

Cauda F, D'Agata F, Sacco K, Duca S, Geminiani G, Vercelli A. 2011. Functional connectivity of the insula in the resting brain. Neuroimage. 55:8-23.

Cavada C, Compañy T, Tejedor J, Cruz-Rizzolo RJ, Reinoso-Suárez F. 2000. The anatomical connections of the macaque monkey orbitofrontal cortex. A review. Cereb Cortex. 10:220-242.

Cerliani L, Thomas RM, Jbabdi S, Siero JCW, Nanetti L, Crippa A, Gazzola V, D’Arceuil H, Keysers C. 2012. Probabilistic tractography recovers a rostrocaudal trajectory of connectivity variability in the human insular cortex. Hum Brain Mapp. 33:2005-2034.

Chikama M, McFarland NR, Amaral DG, Haber SN. 1997. Insular cortical projections to functional regions of the striatum correlate with cortical cytoarchitectonic organization in the primate. J Neurosci. 17:9686-9705.

Chumbley JR, Friston KJ. 2009. False discovery rate revisited: FDR and topological inference using Gaussian random fields. Neuroimage. 44:62-70.

Cloutman LL, Lambon Ralph MA. 2012. Connectivity-based structural and functional parcellation of the human cortex using diffusion imaging and tractography. Front Neuroanat. 6:34.

Cohen AL, Fair DA, Dosenbach NUF, Miezin FM, Dierker D, Van Essen DC, Schlaggar BL, Petersen SE. 2008. Defining functional areas in individual human brains using resting functional connectivity MRI. Neuroimage. 41:45-57.

Cole MW, Yeung N, Freiwald WA, Botvinick M. 2009. Cingulate cortex: diverging data from humans and monkeys. Trends Neurosci. 32:566-574.

Corbetta M, Patel G, Shulman GL. 2008. The reorienting system of the human brain: from environment to theory of mind. Neuron. 58:306 -324 .

Craddock RC, James GA, Holtzheimer PE, Hu XP, Mayberg HS. 2012. A whole brain fMRI atlas generated via spatially constrained spectral clustering. Hum Brain Mapp. 33:1914-1928.

Deen B, Pitskel NB, Pelphrey KA. 2011. Three systems of insular functional connectivity identified with cluster analysis. Cereb Cortex. 21:1498-1506.

Devinsky O, D’Esposito M. 2004. Neurology of cognitive and behavioral disorders. New York: Oxford University Press. 
Drevets WC, Price JL, Furey ML. 2008. Brain structural and functional abnormalities in mood disorders: implications for neurocircuitry models of depression. Brain Struct Funct. 213:93-118.

Duncan J, Owen AM. 2000. Common regions of the human frontal lobe recruited by diverse cognitive demands. Trends Neurosci. 23:475- 483.

Etkin A, Egner T, Kalisch R. 2011. Emotional processing in anterior cingulate and medial prefrontal cortex. Trends Cogn Sci. 15:85-93.

Ferry AT, Ongür D, An X, Price JL. 2000. Prefrontal cortical projections to the striatum in macaque monkeys: evidence for an organization related to prefrontal networks. J Comp Neurol. 425:447-470.

Flynn FG, Benson DF, Ardilas A. 1999. Anatomy of the insula - functional and clinical correlates. Aphasiology. 13:55-78.

Ford A, McGregor KM, Case K, Crosson B, White KD. 2010. Structural connectivity of Broca's area and medial frontal cortex. Neuroimage. 52:1230-1237.

Fortunato S. 2010. Community detection in graphs. Phys Rep. 486:75-174.

Fox MD, Corbetta M, Snyder AZ, Vincent JL, Raichle ME. 2006. Spontaneous neuronal activity distinguishes human dorsal and ventral attention systems. Proc Natl Acad Sci U S A. 103:10046-10051.

Gallay DS, Gallay MN, Jeanmonod D, Rouiller EM, Morel A. 2012. The insula of Reil revisited: multiarchitectonic organization in macaque monkeys. Cereb Cortex. 22:175-190.

Garrett EA, DeLong MR, Strick PL. 1986. Parallel organization of functionally segregated circuits linking basal ganglia and cortex. Ann Rev Neurosci. 9:357-81.

Ghashghaei HT, Hilgetag CC, Barbas H. 2007. Sequence of information processing for emotions based on the anatomic dialogue between prefrontal cortex and amygdala. Neuroimage. 34:905-923.

Gilbert SJ, Spengler S, Simons JS, Steele JD, Lawrie SM, Frith CD, Burgess PW. 2006. Functional specialization within rostral prefrontal cortex (area 10): a meta-analysis. J Cogn Neurosci. 18:932-948.

Girvan M, Newman MEJ. 2002. Community structure in social and biological networks. Proc Natl Acad Sci USA. 99:7821-7826.

Goebel R, Esposito F, Formisano E. 2006. Analysis of functional image analysis contest (FIAC) data with brainvoyager QX: From single-subject to cortically aligned group general linear model analysis and self-organizing group independent component analysis. Hum Brain Mapp. 27:392-401.

Golestani A-M, Goodyear BG. 2011. A resting-state connectivity metric independent of temporal signal-to-noise ratio and signal amplitude. Brain Connect. 1:159-167.

Goulas A, Uylings HBM, Stiers P. 2012. Unravelling the intrinsic functional organization of the human lateral frontal cortex: a parcellation scheme based on resting state fMRI. J Neurosci. 32:10238-10252. 
Groman SM, James AS, Seu E, Crawford MA, Harpster SN, Jentsch JD. 2013. Monoamine levels within the orbitofrontal cortex and putamen interact to predict reversal learning performance. Biol Psychiatry. 73:756-762.

Guimerá R, Amaral LAN. 2005. Functional cartography of complex metabolic networks. Nature. 433:895-900.

Habas C. 2010. Functional connectivity of the human rostral and caudal cingulate motor areas in the brain resting state at 3T. Neuroradiology. 52:47-59.

Haber SN, Kunishio K, Mizobuchi M. 1995. The Orbital and Medial Prefrontal Circuit Through the Primate Basal Ganglia. J Neurosci. 75:4851-4867.

Hof PR, Mufson EJ, Morrison JH. 1995. Human orbitofrontal cortex: cytoarchitecture and quantitative immunohistochemical parcellation. J Comp Neurol. 359:48-68.

Hoffstaedter F, Grefkes C, Caspers S, Roski C, Palomero-Gallagher N, Laird AR, Fox PT, Eickhoff SB. 2013. The role of anterior midcingulate cortex in cognitive motor control: Evidence from functional connectivity analyses. Hum Brain Mapp. 00:1-13.

Holland BS, Copenhaver MD. 1987. An improved sequentially rejective Bonferroni Test procedure. Biometrics. 43:417-423.

Holm S. 1979. A Simple Sequentially Rejective Multiple Test Procedure. Scand J Statist. 6:6570.

Mayberg HS. 2008. Anatomical connectivity of the subgenual cingulate region targeted with deep brain stimulation for treatment-resistant depression. Cereb Cortex. 18:1374-1383.

Kahnt T, Chang LJ, Park SQ, Heinzle J, Haynes J-D. 2012. Connectivity-based parcellation of the human orbitofrontal cortex. J Neurosci. 32:6240-6250.

Kelly C, Uddin LQ, Shehzad Z, Margulies DS, Castellanos FX, Milham MP, Petrides M. 2010. Broca's region: linking human brain functional connectivity data and non-human primate tracing anatomy studies. Eur J Neurosci. 32:383-398.

Kelly C, Toro R, Di Martino A, Cox CL, Bellec P, Castellanos FX, Milham MP. 2012. A convergent functional architecture of the insula emerges across imaging modalities. Neuroimage. 61:1129-1142.

Kim JH, Lee JM, Jo HJ, Kim SH, Lee JH, Kim ST, Seo SW, Cox RW, Na DL, Kim SI, Saad ZS. 2010. Defining functional SMA and pre-SMA subregions in human MFC using resting state fMRI: functional connectivity-based parcellation method. Neuroimage. 49:23752386.

Klein JC, Rushworth MFS, Behrens TEJ, Mackay CE, de Crespigny AJ, D'Arceuil H, Johansen-Berg H. 2010. Topography of connections between human prefrontal cortex and mediodorsal thalamus studied with diffusion tractography. Neuroimage. 51:555564.

Koski R, Paus T. 2000. Functional connectivity of the anterior cingulate cortex within the human frontal lobe : a brain-mapping meta-analysis. Exp Brain Res. 12:55-65. 
Kringelbach ML, Rolls ET. 2004. The functional neuroanatomy of the human orbitofrontal cortex: evidence from neuroimaging and neuropsychology. Prog Neurobiol. 72:341372.

Kringelbach ML. 2005. The human orbitofrontal cortex: linking reward to hedonic experience. Nat Rev Neurosci. 6:691-702.

Krubitzer L. 1995. The organization of neocortex in mammals. Are species differences really so different. TINS 18:408-417.

Kurth F, Zilles K, Fox PT, Laird AR, Eickhoff SB. 2010. A link between the systems: functional differentiation and integration within the human insula revealed by meta-analysis. Brain Struct Funct. 214:519-534.

Lancichinetti A, Fortunato S. 2009. Community detection algorithms: a comparative analysis. Phys Rev E. 80:056117.

Liu H, Qin W, Li W, Fan L, Wang J, Jiang T, Yu C. 2013. Connectivity-based parcellation of the human frontal pole with diffusion tensor imaging. J Neurosci. 33:6782-6790.

Mackey S, Petrides M. 2009. Architectonic mapping of the medial region of the human orbitofrontal cortex by density profiles. Neuroscience. 159:1089-1107.

Mackey S, Petrides M. 2010. Quantitative demonstration of comparable architectonic areas within the ventromedial and lateral orbital frontal cortex in the human and the macaque monkey brains. Eur J Neurosci. 32:1940-1950.

Maldjian JA, Laurienti PJ, Kraft RA, Burdette JH. 2003. An automated method for neuroanatomic and cytoarchitectonic atlas-based interrogation of fMRI data sets. Neuroimage. 19:1233-1239.

Maldjian JA, Laurienti PJ, Burdette JH. 2004. Precentral gyrus discrepancy in electronic versions of the Talairach atlas. Neuroimage. 21:450-455.

Margulies DS, Kelly AMC, Uddin LQ, Biswal BB, Castellanos FX, Milham MP. 2007. Mapping the functional connectivity of anterior cingulate cortex. Neuroimage. 37:579-588.

Margulies DS, Vincent JL, Kelly C, Lohmann G, Uddin LQ, Biswal BB, Villringer A, Castellanos FX, Milham MP, Petrides M. 2009. Precuneus shares intrinsic functional architecture in humans and monkeys. Proc Natl Acad Sci USA. 106:20069 -20074.

Margulies DS, Petrides M. 2013. Distinct parietal and temporal connectivity profiles of ventrolateral frontal areas involved in language production. J Neurosci. 33:1684616852.

Mars RB, Jbabdi S, Sallet J, O’Reilly JX, Croxson PL, Olivier E, Noonan MP, Bergmann C, Mitchell AS, Baxter MG, Behrens TEJ, Johansen-Berg H, Tomassini V, Miller KL, Rushworth MFS. 2011. Diffusion-weighted imaging tractography-based parcellation of the human parietal cortex and comparison with human and macaque resting-state functional connectivity. J Neurosci. 31:4087-4100.

Mars RB, Sallet J, Schüffelgen U, Jbabdi S, Toni I, Rushworth MFS. 2012. Connectivity-based subdivisions of the human right "temporoparietal junction area": evidence for different areas participating in different cortical networks. Cereb Cortex. 22:1894-1903. 
Matelli M, Luppino G. 2001. Parietofrontal circuits for action and space perception in the macaque monkey. Neuroimage. 14:S27-32.

Medford N, Critchley HD. 2010. Conjoint activity of anterior insular and anterior cingulate cortex: awareness and response. Brain Struct Funct. 214:535-549.

Mesulam M-M, Mufson EJ. 1982. Insula of the old world monkey. III: Efferent cortical output and comments on function. J Comp Neurol. 212:38-52.

Meunier D, Lambiotte R, Fornito A, Ersche KD, Bullmore ET. 2009. Hierarchical modularity in human brain functional networks. Front Neuroinform. 3:37.

Miranda-Dominguez O, Mills BD, Grayson D, Woodall A, Grant K a, Kroenke CD, Fair DA. 2014. Bridging the gap between the human and macaque connectome: a quantitative comparison of global interspecies structure-function relationships and network topology. J Neurosci. 34:5552-5563.

Moayedi M, Salomons T V, Dunlop K a M, Downar J, Davis KD. 2014. Connectivity-based parcellation of the human frontal polar cortex. Brain Struct Funct. DOI 10.1007/s00429014-0809-6.

Morecraft RJ, Geula C, Mesulam M-M. 1992. Cytoarchitecture and neural afferents of orbitofrontal cortex in the brain of the monkey. J Comp Neurol. 323:341-358.

Mufson EJ, Mesulam M-M. 1982. Insula of the old world monkey. II: Afferent cortical input and comments on the claustrum. J Comp Neurol. 212:23-37.

Munkres J. 1957. Algorithms for the assignment and transportation problems. J Soc Ind Appl Math. 5:32-38.

Muranishi M, Inokawa H, Yamada H, Ueda Y, Matsumoto N, Nakagawa M, Kimura M.2011. Inactivation of the putamen selectively impairs reward history-based action selection. Exp Brain Res. 209:235-246.

Nelson SM, Dosenbach NUF, Cohen AL, Wheeler ME, Schlaggar BL, Petersen SE. 2010. Role of the anterior insula in task-level control and focal attention. Brain Struct Funct. 214:669-680.

Neubert F-X, Mars RB, Thomas AG, Sallet J, Rushworth MFS. 2014. Comparison of Human Ventral Frontal Cortex Areas for Cognitive Control and Language with Areas in Monkey Frontal Cortex. Neuron. 81:700-713.

Newman MEJ. 2006. Modularity and community structure in networks. Proc Natl Acad Sci USA. 103:8577-8582.

Ongür D, An X, Price JL. 1998. Prefrontal cortical projections to the hypothalamus in macaque monkeys. J Comp Neurol. 401:480-505.

Ongür D, Price JL. 2000. The organization of networks within the orbital and medial prefrontal cortex of rats, monkeys and humans. Cereb Cortex. 10:206-219.

Ongür D, Ferry AT, Price JL. 2003. Architectonic subdivision of the human orbital and medial prefrontal cortex. J Comp Neurol. 460:425-449. 
Palomero-Gallagher N, Mohlberg H, Zilles K, Vogt BA. 2008. Cytology and receptor architecture of human anterior cingulate cortex. J Comp Neurol. 508:906-926.

Palomero-Gallagher N, Zilles K, Schleicher A, Vogt BA. 2013. Cyto- and receptor architecture of area 32 in human and macaque brains. J Comp Neurol. 521:3272-3286.

Pandya DN, Van Hoesen GW, Mesulam M-M. 1981. Efferent connections of the cingulate gyrus in the rhesus monkey. Exp Brain Res. 9:319-330.

Passingham RE, Stephan KE, Kötter R. 2002. The anatomical basis of functional localization in the cortex. Nat Rev Neurosci. 3:606-616.

Petrides M, Pandya DN. 1994. Comparative architectonic analysis of the human and the macaque frontal cortex. In: Handbook of Neuropsychology (Boller F, Grafman J, ed), pp17-58. Amsterdam: Elsevier.

Petrides M, Pandya DN. 2006. Efferent Association Pathways Originating in the Caudal Prefrontal Cortex in the Macaque Monkey. J Comp Neurol. 251:227-251.

Petrides M, Pandya DN. 2012. The frontal cortex. In: The human nervous system (Mai JK, Paxinos G, ed), pp988-1011. Elsevier AP.

Price JL. 2007. Definition of the orbital cortex in relation to specific connections with limbic and visceral structures and other cortical regions. Ann N Y Acad Sci. 1121:54-71.

Price JL, Drevets WC. 2010. Neurocircuitry of mood disorders. Neuropsychopharmacology. 35:192-216.

Price JL, Drevets WC. 2012. Neural circuits underlying the pathophysiology of mood disorders. Trends Cogn Sci. 16:61-71.

Power JD, Barnes KA, Snyder AZ, Schlaggar BL, Petersen SE. 2012. Spurious but systematic correlations in functional connectivity MRI networks arise from subject motion. Neuroimage. 59:2142-2154.

Rajkowska G, Goldman-Rakic PS. 1995. Cytoarchitectonic definition of prefrontal areas in the normal human cortex. I. Remapping of areas 9 and 46 using quantitative criteria. Cereb Cortex. 5:307-322.

Rolls ET, Grabenhorst F. 2008. The orbitofrontal cortex and beyond: from affect to decisionmaking. Prog Neurobiol. 86:216-244.

Rolls ET. 2014. Emotion and Decision-Making Explained. Oxford University Press: Oxford.

Rorden C, Brett M. 2000. Stereotaxic display of brain lesions. Behav Neurol. 12:191-200.

Ross RS, Lopresti ML, Schon K, Stern CE. 2013. Role of the hippocampus and orbitofrontal cortex during the disambiguation of social cues in working memory. Cogn Affect Behav Neurosci. 13:900-915.

Rubinov M, Sporns O. 2010. Complex network measures of brain connectivity: uses and interpretations. Neuroimage. 52:1059-1069. 
Rudebeck PH, Murray EA. 2011. Balkanizing the primate orbitofrontal cortex: distinct subregions for comparing and contrasting values. Ann N Y Acad Sci. 1239:1-13.

Rushworth MFS, Behrens TEJ, Rudebeck PH, Walton ME. 2007. Contrasting roles for cingulate and orbitofrontal cortex in decisions and social behaviour. Trends Cogn Sci. 11:168-176.

Rushworth MFS, Noonan MP, Boorman ED, Walton ME, Behrens TE. 2011. Frontal cortex and reward-guided learning and decision-making. Neuron. 70:1054-1069.

Rushworth MFS, Mars RB, Sallet J. 2013. Are there specialized circuits for social cognition and are they unique to humans? Curr Opin Neurobiol. 23:436-442.

Saleem KS, Miller B, Price JL. 2014. Subdivisions and connectional networks of the lateral prefrontal cortex in the macaque monkey. J Comp Neurol. 522:1641-1690.

Sallet J, Mars RB, Noonan MP, Neubert F-X, Jbabdi S, O'Reilly JX, Filippini N, Thomas AG, Rushworth MF. 2013. The organization of dorsal frontal cortex in humans and macaques. J Neurosci. 33:12255-12274.

Sarkisov SA, Filimonov IN, Kononova EP, Preobraschenskaja IS, Kukuev LA. 1955. Atlas of cytoarchitectonics of the human cerebral cortex. Medzig, Moscow.

Seeley WW, Menon V, Schatzberg AF, Keller J, Glover GH, Kenna H, Reiss AL, Greicius MD. 2007. Dissociable intrinsic connectivity networks for salience processing and executive control. J Neurosci. 27:2349-2356.

Selemon LD, Goldman-Rakic PS. 1985. Longitudinal topography and interdigitation of corticostriatal projections in the rhesus monkey. J Neurosci. 5:776-794.

Shen X, Papademetris X, Constable RT. 2010. Graph-theory based parcellation of functional subunits in the brain from resting-state fMRI data. Neuroimage. 50:1027-1035.

Smits M, Peeters RR, van Hecke P, Sunaert S. 2007. A 3 T event-related functional magnetic resonance imaging (fMRI) study of primary and secondary gustatory cortex localization using natural tastants. Neuroradiology. 49:61-71.

Stiers P, Mennes M, Sunaert S. 2010. Distributed task coding throughout the multiple demand network of the human frontal-insular cortex. Neuroimage. 52:252-262.

Talairach T, Tournoux P. 1988. Co-planar stereotaxic atlas of the human brain. Thieme Medical Publishers Inc: New York.

Taylor KS, Seminowicz DA, Davis KD. 2009. Two systems of resting state connectivity between the insula and cingulate cortex. Hum Brain Mapp. 30:2731-2745.

Timbie C, Barbas H. 2014. Specialized pathways from the primate amygdala to posterior orbitofrontal cortex. J Neurosci. 34:8106-8118.

Tomassini V, Jbabdi S, Klein JC, Behrens TEJ, Pozzilli C, Matthews PM, Rushworth MFS, Johansen-Berg H. 2007. Diffusion-weighted imaging tractography-based parcellation of the human lateral premotor cortex identifies dorsal and ventral subregions with anatomical and functional specializations. J Neurosci. 27:10259-10269. 
Torta DME, Costa T, Duca S, Fox PT, Cauda F. 2013. Parcellation of the cingulate cortex at rest and during tasks: a meta-analytic clustering and experimental study. Front Hum Neurosci. 7:275.

Triantafyllou C, Polimeni JR, Wald LL. 2011. Physiological noise and signal-to-noise ratio in fMRI with multi-channel array coils. Neuroimage. 55:597-606.

Tziortzi AC, Searle GE, Tzimopoulou S, Salinas C, Beaver JD, Jenkinson M, Laruelle M, Rabiner EA, Gunn RN. 2011. Imaging dopamine receptors in humans with [11C]-(+)PHNO: dissection of D3 signal and anatomy. Neuroimage. 54:264-277.

Tzourio-Mazoyer N, Landeau B, Papathanassiou D, Crivello F, Etard O, Delcroix N, Mazoyer B, Joliot M. 2002. Automated anatomical labeling of activations in SPM using a macroscopic anatomical parcellation of the MNI MRI single-subject brain. Neuroimage. 15:273-289.

Uylings HBM, Sanz-Arigita EJ, de Vos K, Pool CW, Evers P, Rajkowska G. 2010. 3-D cytoarchitectonic parcellation of human orbitofrontal cortex correlation with postmortem MRI. Psychiatry Res. 183:1-20.

Van Dijk KRA, Hedden T, Venkataraman A, Evans KC, Lazar SW, Buckner RL. 2010. Intrinsic functional connectivity as a tool for human connectomics: theory, properties, and optimization. J Neurophysiol. 103:297-321.

Van Dijk KRA, Sabuncu MR, Buckner RL. 2012. The influence of head motion on intrinsic functional connectivity MRI. Neuroimage. 59:431-438.

Von Economo C, Koskinas GN. 1925. Die Cytoarchitektonik der Hirnrinde des Erwachsenen Menschen. Springer Verlag, Berlin.

Van Eijndhoven P, Van Wingen G, Katzenbauer M, Groen W, Tepest R, Fernández G, Buitelaar J, Tendolkar I. 2013. Paralimbic Cortical Thickness in First-Episode Depression: Evidence for Trait-Related Differences in Mood Regulation. Am J Psychiat. 170:1477-1486.

Vogt BA, Pandya DN. 1987. Cingulate cortex of the rhesus monkey. II: Cortical afferents. J Comp Neurol. 262:271-289.

Vogt BA. 2005. Pain and emotion interactions in subregions of the cingulate gyrus. Nat Rev Neurosci. 6:533-544.

Vogt BA, Vogt L, Farber NB, Bush G. 2005. Architecture and neurocytology of monkey cingulate gyrus. J Comp Neurol. 485:218-239.

Vogt BA, Hof PR, Zilles K, Vogt LJ, Herold C, Palomero-Gallagher N. 2013. Cingulate area 32 homologies in mouse, rat, macaque and human: cytoarchitecture and receptor architecture. J Comp Neurol. 521:4189-4204.

Wallis JD. 2012. Cross-species studies of orbitofrontal cortex and value-based decisionmaking. Nat Neurosci. 15:13-19.

Yeo BT, Krienen FM, Sepulcre J, Sabuncu MR, Lashkari D, Hollinshead M, Roffman JL, Smoller JW, Zöllei L, Polimeni JR, Fischl B, Liu H, Buckner RL. 2011. The organization 
of the human cerebral cortex estimated by functional connectivity. J Neurophysiol. 106:1125-1165.

Yeterian EH, Pandya DN, Tomaiuolo F, Petrides M. 2012. The cortical connectivity of the prefrontal cortex in the monkey brain. Cortex. 48:58-81.

Yu C, Zhou Y, Liu Y, Jiang T, Dong H, Zhang Y, Walter M. 2011. Functional segregation of the human cingulate cortex is confirmed by functional connectivity based neuroanatomical parcellation. Neuroimage. 54:2571-2581.

Zalesky A, Fornito A, Bullmore E. 2012. On the use of correlation as a measure of network connectivity. Neuroimage. 60:2096-2106.

Zou Q, Long X, Zuo X, Yan C, Zhu C, Yang Y, Liu D, He Y, Zang Y. 2009. Functional connectivity between the thalamus and visual cortex under eyes closed 
Chapter 3

Orbitomedial prefrontal cortex functional connectivity markers of Major

Depression vulnerability and disease

Zoe Samara, Elisabeth A.T. Evers, Frenk Peeters, Harry B.M. Uylings, Grazyna Rajkowska, Johannes G. Ramaekers and Peter Stiers 


\section{Abstract}

Pathophysiology models of major depressive disorder or major depression (MD) center on the dysfunction of various cortical areas within the orbitomedial prefrontal cortex (OMPFC) such as the subgenual and rostral cingulate, orbitofrontal regions and the anterior insula. While independent structural and functional abnormalities in these areas are consistent findings in MD, the complex interactions among them and the rest of the cortex remain largely unexplored. We used resting state functional magnetic resonance imaging (MRI) connectivity to systematically map alterations in the communication between 19 OMPFC cortical fields and the rest of the brain in MD. OMPFC fields were functionally-defined subregions in a parcellation map, previously delineated in a data-driven, objective way using the graph theory metric of modularity. Functional connectivity (FC) maps of each field from participants with current $M D(N=35)$, unaffected first-degree relatives $(\mathrm{N}=38)$ and healthy controls $(\mathrm{N}=38)$ were subjected to conjunction analyses. In this way we distinguished between FC changes common to both patients and at-risk individuals (markers of MD vulnerability) and FC changes unique to the acute MD phase (markers of MD disease). Neuroimaging FC abnormalities in MD vulnerability were found for dorsal medial wall regions and the anterior insula and concerned aberrant communication of these areas with the inferior parietal cortex and dorsal posterior cingulate, occipital areas and the brainstem. FC aberrations in current MD included the anterior insula, rostral and dorsal anterior cingulate and lateral orbitofrontal areas and concerned aberrant communication with the dorsal striatum, the cerebellum, the precuneus, the anterior PFC, somato-motor cortex, DLPFC, and visual areas in the occipital and inferior temporal lobes. Functionally delineated parcellation maps can be used to identify putative connectivity biomarkers in extended cortical regions such as the OMPFC. FC abnormalities of the rostral cingulate and anterior insula characterize both the MD vulnerability and the depressed state. These areas are thus central in the pathophysiology of MD. 


\section{Introduction}

Major Depression (MD) is a debilitating syndrome presenting with various symptoms in mood, reward and decision making (APA, 1994). The efficacy of MD neuro-modulation therapies such as transcranial magnetic stimulation (TMS) and deep brain stimulation (DBS) with various targets demonstrates in a "proof of concept" manner that MD is a systems-level disorder involving a complex pattern of altered interactions between several brain areas (Downar and Daskalakis, 2013; Riva-Posse et al., 2013). Convergent neuroimaging findings suggest that, together with limbic and autonomic centers, subregions within the orbitomedial prefrontal cortex (OMPFC) such as the subgenual, rostral and dorsal cingulate, dorsomedial and dorsolateral PFC areas, the anterior insula and the orbitofrontal cortex (Price and Drevets, 2010; Pizzagalli, 2010; Hamani et al., 2010) play a central role in MD's aberrant networks. Understanding of the neuro-circuitry pathways underlying MD symptoms and treatment response remains elusive, despite the large body of evidence documenting independent abnormalities in various OMPFC subregions and the efficacy of connectivity-based treatments with OMPFC targets.

Elucidation of the functional wiring between the OMPFC cortical fields and key brain areas for autonomic, cognitive and affective control is critical for generating new hypotheses on MD disease mechanisms and identifying connectivity biomarkers to guide diagnosis and treatment. Thus far, MD studies have examined OMPFC FC either only for select coordinates based on activation studies or in a coarse way, as either part of the anterior default mode network (DMN) or with predefined general anatomical labels (Greicius et al., 2007; Guo et al., 2014; Sheline et al., 2010; Wang et al., 2012; Sundermann and Pleiderer 2014). On the theoretical side, neuro-circuit models of MD propose pathways of altered communication between the various components based on cross-species tracer findings or lesion and post mortem human reports (Price and Drevets, 2012).

Recent neuroimaging advances on MRI connectivity and the novel mathematical tools of graph theory enable us to study the cortical organization within a specified area of the cerebrum in vivo. Graph-theory considers the brain a small-world network in which the interplay between local segregation and global integration in cortical organization is reflected by the statistical parameter of modularity (Girvan and Newman, 2002; Rubinov and Sporns, 2010). Using modularity (i.e. quantifying the number of functional connections within a computational unit versus its connections with other units) algorithms such as the Louvain module-optimization create maps of functional cortical fields which can be used to study their neuro-circuit with the rest of the cortex (Barnes et al., 2010; Goulas et al., 2012). This approach has been shown to delineate neuroanatomically plausible subdivisions of the human cortex which closely match cytoarchitectonic and tracing findings and has been used to parcellate the basal ganglia and the 
lateral prefrontal cortex (Barnes et al., 2010; Goulas et al., 2012) in healthy controls.

This study takes advantage of the fully data-driven and unbiased method of MRI cortical mapping to systematically examine FC impairments in a cortical region critical for MD. We use a parcellation map of the lefthemisphere OMPFC consisting of 19 cortical fields (including areas covering the orbital surface, the ventral anterior insula and the anterior cingulate and medial frontal cortex) delineated with resting state functional connectivity (FC) by the Louvain algorithm in healthy controls (Samara et al, submitted). These OMPFC subregions have been shown to exhibit whole-brain (i.e. cortical and subcortical) FC profiles highly similar to macaque anatomical and human MRI connectivity fingerprints (Samara et al., submitted). Their validity was further demonstrated by hierarchical clustering which revealed that they are organized in two extended groups, a medial and an orbital one, in line with previous reports on the cytoarchitectural and connectional organization of the OMPFC (Barbas and Pandya, 1989; Ongür and Price, 2000; Yeterian et al., 2012).

Our aim was to identify FC changes associated with MD between the 19 OMPFC functional subregions delineated in the data-driven map and the rest of the cortex. We sought specifically to test the hypotheses that OMPFC subregions show aberrant connectivity patterns which are: 1) common in MD patients and in at-risk individuals compared to healthy controls (connectivity abnormalities of MD vulnerability), and 2) unique in the MD group compared to at-risk individuals and controls (connectivity markers of acute MD). Finally, we assessed the congruency between self-report measures of MD symptomatology and the FC abnormalities in the acute MD phase.

\section{Materials \& Methods}

\section{Design \& Participants}

Thirty-five MD participants currently experiencing a major depressive episode (cutoff BDI-II > 20; mean age 38.9; SD 11.6; 71\% female), thirty-six unaffected first-degree relatives (mean age 34; SD 14.6; $72 \%$ female) and thirty-eight healthy controls (mean age 36 ; SD $16.4 ; 68 \%$ female) participated in the study after providing informed consent. Patients were included if they met DSM-IV-TR criteria for major depressive disorder and excluded if they were diagnosed with Bipolar I or II, substance dependence or were taking benzodiazepines. Participants in the other two groups were excluded in the presence of any axis I diagnosis. Recruitment and screening procedures for all participants and diagnostic and medication histories of patients are reported in the Supplement. The study was approved by the Medical Ethical Committee of Maastricht University Medical Center. 


\section{Clinical Assessments}

During testing, participants completed the following self-report scales: Beck Depression Inventory-II (BDI-II), Brief Symptom Inventory (BSI), and the Quick Inventory of Depressive Symptomatology Self-Report (QIDS-SR) (Beck et al., 1996; Derogatis and Melisaratos, 1983; Rush et al., 2003). Participants were also assessed with a computerized version of Raven's Standard Progressive Matrices, a 60-item test of non-verbal IQ (Raven et al., 2003).

\section{Magnetic Resonance Imaging Acquisition \& Preprocessing}

Magnetic resonance imaging data included a resting state scan (repetition time=2.5 sec, 203 volumes; optimized for reduction of susceptibility artefacts), a high-resolution T1-weighted anatomical scan and a field map. Data were preprocessed with SPM 5 (Welcome Trust Center for Neuroimaging) for slice time and motion correction, spatial correction with the field map, smoothing, normalization to the Montreal Neurological Institute 152 template, detrending, removal of nuisance signals and Fourier band pass filtering. To eliminate the detrimental effect of head motion on our FC metric (Van Dijk et al., 2012) and exclude the possibility our results could be explained by between-group differences in frame-wise displacement (Power et al., 2012) we removed movement-contaminated volumes and matched the three groups with respect to the mean frame-wise relative translation distance present after volume removal. Acquisition parameters and preprocessing steps are reported in detail in the Supplement.

\section{Statistical Analyses}

We sought to identify FC abnormalities in 19 OMPFC cortical fields of the left-hemisphere parcellation map constructed in a previous study (Samara et al., submitted). Individual resting-state FC datasets from healthy controls $(\mathrm{N}=34)$ were partitioned into OMPFC regions by the Louvain modularity optimization algorithm. The group parcellation map (see Figure $1 \mathrm{~A}$ of Chapter 2; pp. 23) was created using a clustering method based on spatial proximity. Methods of the parcellation study are reported in the Supplement.

To systematically examine connectivity changes in each OMPFC cortical field associated with MD, we generated seed-based whole-brain FC maps for each of the 19 subregions. As seeds we used the center of mass (COM) (i.e. the average MNI position across all voxels) of each subregion (see Table 2 of Chapter 2; pp.35 for each field's MNI coordinates and putative correspondence with cytoarchitectonic nomenclature). The timecourse of each seed was de-noised (Eigen decomposition) before calculating Pearson's $r$ between each seed's time-course and the time-course of all voxels in the brain. Values in the whole-brain FC maps were $r$ - to-Z transformed using Fisher's formula. Subsequently, the FC maps of OMPFC 
field were subjected to an analysis of covariance (ANCOVA) with group as a factor and age, gender and IQ Raven score as covariates.

To test for connectivity abnormalities of MD vulnerability, we created conjunction maps of the F contrasts between 1) the healthy controls and the MD group and 2) the healthy controls and the at-risk participants.

Conjunction maps were tested for significance with Monte Carlo simulations (10.000 iterations; voxel-level $p=.01$, cluster-level $p=.0025$ - Bonferroniadjusted for 19 independent comparisons). To retain in the analysis only brain areas with altered FC in both the MD and the vulnerability group, we removed from the conjunction maps all voxels significant at a lenient threshold (.001 uncorrected) in the $F$ contrast between MD patients versus at-risk participants.

To test for connectivity abnormalities in acute MD, we created conjunction maps of the F contrasts between 1) the MD group and healthy controls and 2) MD patients and at-risk individuals. Conjunction maps were tested for significance with Monte Carlo simulations (10.000 iterations; voxellevel $p=.01$, cluster-level $p=.0025$ - Bonferroni-adjusted for 19 independent comparisons). To retain in the analysis only brain areas with altered FC in exclusively the disease state, we removed from the conjunction maps all voxels significant at a lenient threshold (.001 uncorrected) in the contrast between at-risk participants versus controls.

Since ANOVA is a general test for differences between groups, we determined the direction of FC differences in MD vulnerability and disease with ROI-based post-hoc tests in SPSS using the r-Z-transformed connectivity values of the statistically significant voxels in the conjunction maps. To relate our FC findings to disease variables, correlation analyses for the MD group between the FC changes in the acute phase and our clinical measures were run in SPSS (IBM SPSS 21). For parameters and details of all analyses see Supplement.

Finally, to ensure that the connectivity differences reported were not driven by antidepressant medication use and comorbidity in our MD sample or gray matter differences between the three groups, we removed from our results maps: a) voxels in which FC was significantly different at a lenient threshold (.001 uncorrected) between medicated versus non-medicated MD participants and between MD participants with and without axis I comorbidity, and b) all voxels which their probability of being gray matter was significantly different (.001 uncorrected) among the groups.

\section{Results}

\section{Demographic and Clinical Characteristics}

MD patients had their first episode on average at the age of 29.3 $(S D=11.4)$ and a mean of 2.9 episodes $(S D=3)$. Comorbidity (having at least one additional axis I diagnosis) and use of antidepressants at the time of the 
scan were reported by $51 \%$ and $48 \%$ of the MD group respectively. Most prevalent comorbid diagnosis was social phobia and the majority of participants were using SSRI's (Supplementary Table 1). Average BDI-II and QIDS-SR scores at the time of the scan in the MD group were 32.74 and 16.57 respectively. While at-risk participants had clinically and statistically significantly lower scores of depressive and general psychiatric symptomatology compared to the MD group, they scored fairly higher compared to healthy controls (Table 1). Groups were matched in age, gender and IQ (all p's >.2).

Table 1. Sample characteristics and clinical measures.

$$
\text { MD } \quad \text { FH HC }
$$

\begin{tabular}{lccccccccc} 
& Mean & SD & Mean & SD & Mean & SD & $\mathbf{F}(2,106)$ & \multicolumn{2}{c}{ p Values } \\
BDI-II & 32.74 & 7.66 & 5.78 & 5.04 & 2.24 & 2.97 & 328.68 & $.000^{\mathrm{a}}$ & $.020^{\mathrm{b}}$ \\
BSI GSI & 1.67 & 0.55 & 0.34 & 0.38 & 0.14 & 0.17 & 159.24 & $.000^{\mathrm{a}}$ & $.098^{\mathrm{b}}$ \\
$\begin{array}{l}\text { BSI } \\
\text { PST }\end{array}$ & 38.66 & 7.67 & 12.42 & 10.16 & 6.5 & 7.27 & 147.18 & $.000^{\mathrm{a}}$ & $.010^{\mathrm{b}}$ \\
$\begin{array}{l}\text { BSI } \\
\text { PSDI }\end{array}$ & 2.26 & 0.46 & 1.18 & 0.39 & 0.89 & 0.41 & 108.07 & $.000^{\mathrm{a}}$ & $.009^{\mathrm{b}}$ \\
QIDS & 16.57 & 3.38 & 3.91 & 3.46 & 2.1 & 1.84 & 252.58 & $.000^{\mathrm{a}}$ & $.030^{\mathrm{b}}$ \\
$\begin{array}{l}\text { Age } \\
\text { \% }\end{array}$ & 38.88 & 11.8 & 34.03 & 14.77 & 36.21 & 16.65 & 0.98 & .377 & \\
$\begin{array}{l}\text { Female } \\
\text { Raven }\end{array}$ & $71 \%$ & --- & $72 \%$ & --- & $68 \%$ & --- & $X^{2}(2)=$ & .930 & \\
IQ & 45.2 & 8.99 & 48.28 & 6.5 & 46.03 & 7.46 & 1.53 & .221 &
\end{tabular}

$M D$, patient group; $F H$, at-familial risk group; $H C$, healthy controls; GSI, global severity index; PST, positive symptom total; PSDI, positive symptom distress index. ${ }^{a}$ The $M D$ group differs significantly from relatives and controls; ${ }^{b}$ The $F H$ group differs significantly from controls.

\section{Neuroimaging Analysis}

\section{FC abnormalities of MD Vulnerability}

In our analysis for aberrations in MD vulnerability we found that MD patients and at-risk individuals share various FC abnormalities in the OMPFC compared to controls. ANOVA's ( $F$ maps cluster-based corrected using Monte Carlo and voxel-level $p=.01$; cluster-level $p=.0026$ ) revealed changes in four OMPFC subregions: the anterior insula, cingulate regions $p 32$ and d32 and dorsomedial frontal area BA9 (Figure 1). ROI-based post-hoc 
analysis indicated that MD vulnerability is characterized by decreased FC between area BA9 and a lateral occipital region and between area p32 and dorsal posterior cingulate and inferior parietal cortex (see Table 2 for $p$ values of post-hoc tests). In contrast, increased FC was observed between the anterior insula and the brainstem (PAG/dorsal raphe) and between medial region $\mathrm{d} 32$ and the lateral occipital cortex.
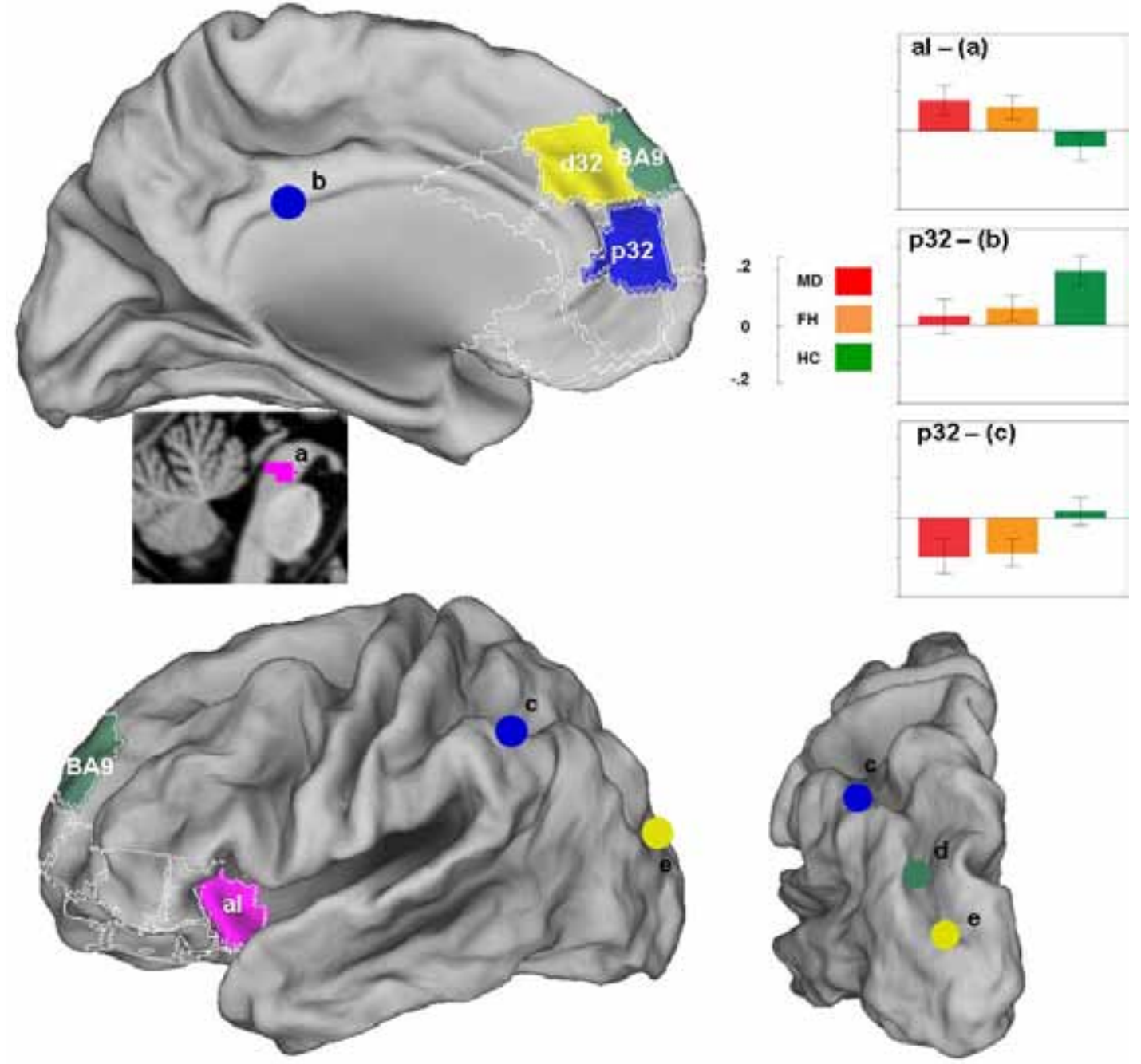

Figure 1. Functional connectivity abnormalities of MD Vulnerability. Region nomenclature corresponds to Figures and Table 2 of Chapter 2 (pp.35). Spheres are colored according to the OMPFC region they were found to have altered connectivity. Bar plots are shown for the anterior insula (al) and the rostral cingulate (p32) (see Supplement for all plots). Values in plots are Z- transformed correlations and error bars represent $95 \%$ confidence intervals. MD, patient group; FH, at-risk group; HC, healthy controls. Spheres $c$ and e concern the right hemisphere. 


\section{FC abnormalities of MD Disease}

In our analysis for aberrations in MD disease, we found that MD patients, in addition to the FC changes they share with the at-risk group, show unique impairments in the communication of the OMPFC with the rest of the cortex. ANOVA's revealed changes in six OMPFC subdivisions: the anterior insula, anterior cingulate regions p32 and p24, mid-cingulate a24' and orbitofrontal areas 11 and $47 / 12$. Specifically, MD disease abnormalities concerned altered communication between the anterior insula and the left putamen, the right caudate, and left fusiform gyrus and cerebellum; between p32 and the precuneus; between lateral orbital area 47/12 and the lateral occipital cortex bilaterally, the left inferior temporal lobe and the parietal paracentral lobule; between central orbital area 11 and area $7 \mathrm{~m}$ on the dorsal precuneus; between area p24 and the anterior PFC; and between area a24' and the dorsolateral PFC and left para-central lobule (Figure 2 and Table 2 for anatomical locations and MNI coordinates). ROI-based post-hoc analysis indicated that MD patients had decreased FC between area p32 and the precuneus compared to both at-risk individuals and controls. In contrasts, the MD group had increased FC compared to both groups between all the other OMPFC cortical fields and the cortical and subcortical regions reported. 


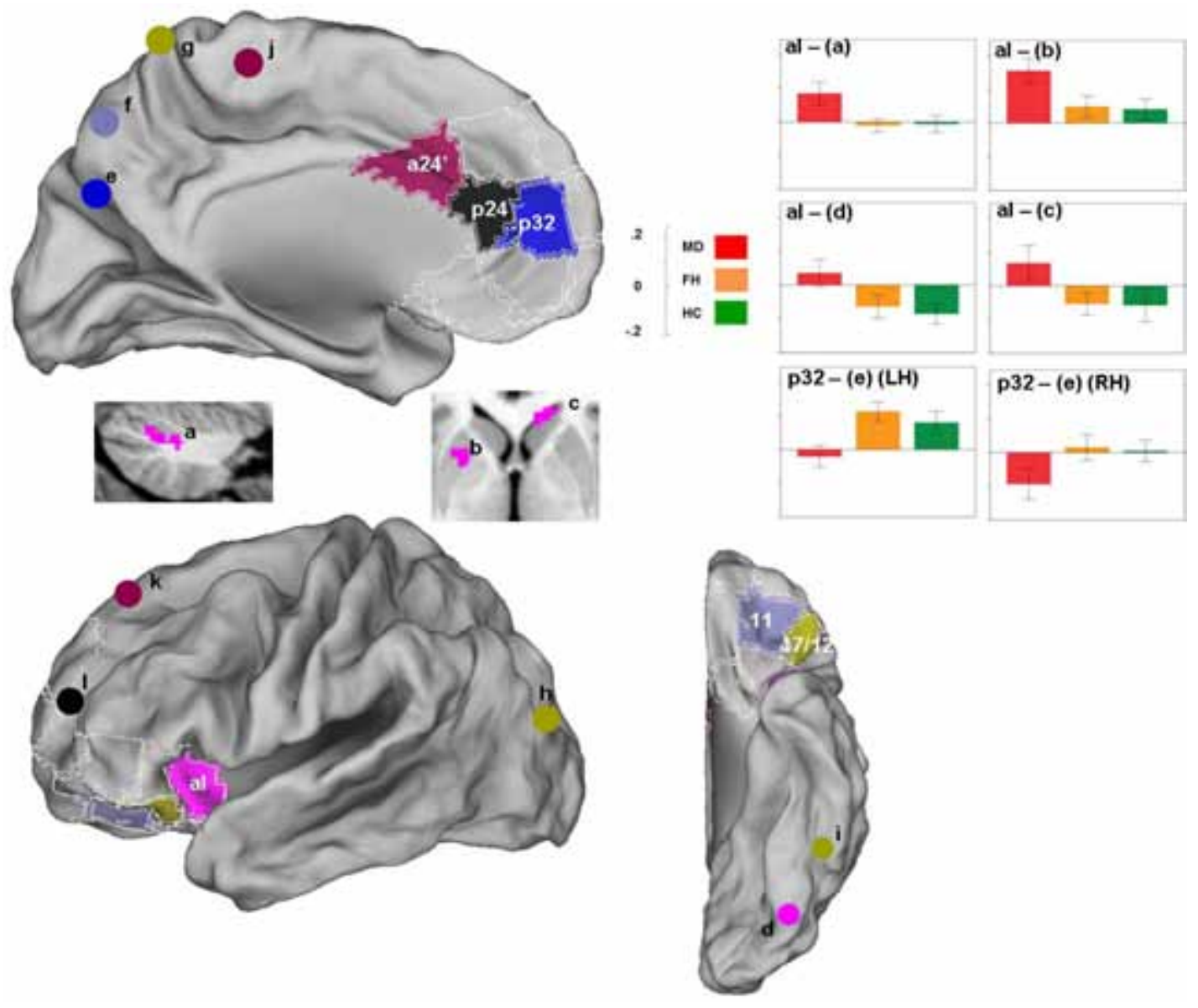

Figure 2. Functional connectivity abnormalities of MD Disease. Region nomenclature corresponds to Figures and Table 2 of Chapter 2 (pp.35). Spheres are colored according to the OMPFC region they were found to have altered connectivity. Bar plots are shown for the anterior insula (al) and the rostral cingulate (p32) (see Supplement for all plots). Values in plots are Z- transformed correlations and error bars represent $95 \%$ confidence intervals. MD, patient group; FH, at-risk group; HC, healthy controls. Spheres a and $i$ concern the right hemisphere.

\section{Correlation Analyses}

Correlation analyses between the self-report measures of depressive symptomatology of the MD group and the connectivity aberrations of the acute phase did not reveal any significant relationships. 
Table 2. Summary of MRI results. Region nomenclature corresponds to Figures and Table 2 of Chapter 2 (pp.35). Anatomy labels are given according to Automated Anatomical Labeling (Tzourio-Mazoyer et al., 2002) and $p$ values concern the overall comparisons (see Supplement for all p's).

\begin{tabular}{|c|c|c|c|c|c|}
\hline Map & OMPFC area & Anatomy & Average MNI & $\begin{array}{l}\text { Voxel } \\
\text { Extent }\end{array}$ & p Value \\
\hline MD Vulnerability & Ant. Insula & Brainstem, PAG & $3,-26,-15$ & 73 & .000005 \\
\hline MD Vulnerability & p32 & Posterior cingulate & $1,-30,30$ & 61 & .000048 \\
\hline MD Vulnerability & p32 & BA 40/39 & $44,-50,45$ & 61 & .000027 \\
\hline MD Vulnerability & BA9 & Intraparietal sulcus & $-30,-72,32$ & 69 & .000001 \\
\hline MD Vulnerability & d32 & Occipital & $23,-91,20$ & 82 & .000001 \\
\hline MD Disease & 47/12 (OFC) & Inferior temporal & $48,-46,-18$ & 53 & .000001 \\
\hline MD Disease & 47/12 (OFC) & Occipital (RH) & $26,-92,20$ & 61 & .000003 \\
\hline MD Disease & $47 / 12$ (OFC) & Occipital (LH) & $-25,-87,21$ & 90 & .000001 \\
\hline MD Disease & $47 / 12$ (OFC) & Paracentral Lobule & $2,-47,73$ & 62 & .000003 \\
\hline MD Disease & Ant. Insula & Cerebellum & $29,-61,-36$ & 76 & .000001 \\
\hline MD Disease & Ant. Insula & Fusiform & $34,-72,-18$ & 93 & .000006 \\
\hline MD Disease & Ant. Insula & Putamen & $-23,5,5$ & 68 & .000002 \\
\hline MD Disease & Ant. Insula & Caudate & $13,25,2$ & 61 & .000538 \\
\hline MD Disease & p32 & Precuneus (LH) & $-8,-72,33$ & 60 & .000000 \\
\hline MD Disease & p32 & Precuneus $(\mathrm{RH})$ & $11,-71,41$ & 69 & .000130 \\
\hline MD Disease & p24 & aPFC & $-27,58,15$ & 72 & .000269 \\
\hline MD Disease & $a 24^{\prime}$ & DLPFC & $-10,42,47$ & 68 & .000037 \\
\hline MD Disease & a24' & Paracentral Lobule & $-7,-25,63$ & 58 & .000008 \\
\hline MD Disease & 11 (OFC) & Precuneus & $4,-66,52$ & 58 & .000929 \\
\hline
\end{tabular}




\section{Discussion}

This was the first study to systematically examine FC abnormalities in cortical fields within the OMPFC in MD. By comparing MD patients, atfamilial-risk participants and healthy controls, we distinguished between the FC alterations that confer vulnerability to MD and the FC correlates of the current episode. Our results suggest that individuals with genetic MD vulnerability, in the absence of clinically significant symptoms, share with patients connectivity aberrations between a number of OMPFC fields and the rest of the brain. The fields involved in MD vulnerability are located in the rostral cingulate, the dorsomedial frontal cortex and the anterior insula and have altered communication with the posterior cingulate, the inferior parietal cortex, lateral occipital areas and the periaqueductal gray. MD patients, in addition to the vulnerability changes, exhibit unique FC alterations in areas of the dorsal cingulate and the orbitofrontal cortex during their MD episode. These regions are differentially connected with various cortical and subcortical centers. Thus, neurobiologically MD starts off with confined predisposing FC abnormalities of the medial PFC and the insula and progresses to a disease state with extensive OMPFC and insula alterations (Figure 3).
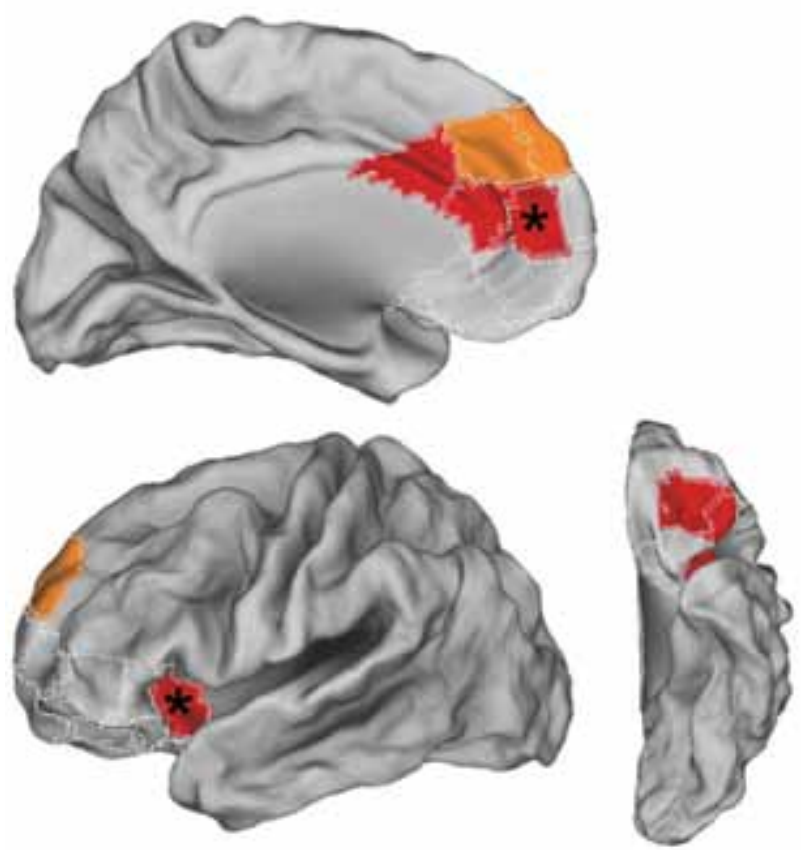

Figure 3. Summary representation of all connectivity findings. OMPFC regions in orange were found to have altered $F C$ in $M D$ vulnerability, while regions in red were found to have altered FC in the acute MD phase. The anterior insula and the rostral 
cingulate (marked with asterisk) are reported to have changed connectivity both in vulnerability and during depressive episodes.

The current results implicate OMPFC cortical fields within the anterior default mode network (DMN) in MD pathology, in line with previous reports of MRI connectivity abnormalities of the DMN (Greicius et al., 2007; Sheline et al., 2010; Sambataro et al., 2013), involved in self-referential processing (Sambataro et al., 2013). At the same time our findings indicate that altered communication is not confined to pairs of regions within the DMN. We observed FC abnormalities between DMN regions and areas of the frontoparietal and visual networks in the posterior brain and between cortical regions within the frontoparietal network (Yeo et al., 2011), i.e. central OFC area 11 and area $7 \mathrm{~m}$ of the dorsal precuneus (Buckner et al., 2008; Yeo et al., 2011). Thus, our results suggest that MD entails more than the perturbation of one large-scale, homogenously altered network; it is a complex pattern of connectivity disturbances within and between neural systems. This is a timely observation given that both DMN heterogeneity (Dastjerdi et al., 2011; Li et al., 2013; Manoliu et al., 2013) and the implication of disturbed communication across large-scale brain networks is increasingly recognized in MD (Marchetti et al., 2012; van Tol et al., 2013; Jacobs et al., 2014; Liston et al., 2014 ).

Our connectivity observations have important implications for understanding MD pathophysiology. The involvement of most of the brain areas we report here having altered FC in MD has been long known. The limbic-cortical dysregulation model of MD, based on metabolic data from PET studies, (Seminowicz et al., 2004) includes, among others, the medial and lateral PFC, the anterior and posterior cingulate, the anterior insula, the parietal cortex, the striatum and the midbrain in sensory, emotional, cognitive, and autonomic integration and the setting of mood. What is largely still unknown however is how exactly these areas interact to give rise to depressive symptomatology. Here we elucidate the specific links that get disrupted among these regions and distinguish the pathways where disturbance of connectivity precedes the onset of clinical symptoms from those where altered communication is either the endophenotype of the depressed state or a post disease-onset change. Among the various cortical regions implicated in MD, rostral cingulate and anterior insula appear to play a central role in MD. Rostral anterior cingulate and anterior insula are implicated in MD diathesis (Pezawas et al., 2005; Boes et al., 2008; Cannon et al., 2007; Takahashi et al., 2010), and MD phenomenology and antidepressant treatment response (Pizzagalli, 2010; Fu et al., 2013). The current study demonstrates that the functional connectivity of both areas is altered in vulnerability and disease states and elaborates the cascade of FC changes originating from these regions. 


\section{Functional connectivity of the rostral cingulate in vulnerability and disease}

Disturbance of $\mathrm{FC}$ between the rostral cingulate and the inferior parietal and dorsal posterior cingulate cortex in MD vulnerability likely represents a dysfunctional pathway of attentional and autonomic control. Behaviorally, disturbances of attentional and autonomic control manifest as difficulties directing attention away from negative stimuli (Browning et al., 2012), reappraising (Viviani, 2013) or suppressing negative distractors (Kerr et al., 2005) and in thus regulating emotional arousal. Such disturbances are considered risk factors in MD, observed in people with genetic susceptibility and remitted depressed (Folland-Ross and Gotlib, 2012; Browning et al., 2012). The rostral cingulate is an area at the intersection of the cognitive and affective ACC subdivisions (Bush et al., 2000) with connections to autonomic centers (Porro et al., 2003; Critchley, 2005). It has been implicated in the assessment of the salience of emotional and motivational information, attentional and conflict monitoring (Folland-Ross and Gotlib, 2012), particularly in the presence of emotional distractors (Bush et al., 2000; Etkin et al., 2006; Roy et al., 2012; Egner et al., 2008; Salomons et al., 2004; Rey et al., 2014) as well as the regulation of emotional responses (Bush et al., 2000; Herwig et al., 2007; Mayberg 2003). The inferior parietal cortex appears also involved in attention-related functions such as sustained attention (Singh-Curry et al., 2009), computation of priorities for attentional allocation (Caspers et al., 2011; Caspers et al., 2013), re-evaluation of conflicting choices between options (Kim et al., 2010; Mars et al., 2011), and the reappraisal/suppression of emotion (Viviani, 2013). Interestingly, inferior parietal cortex has been found to respond primarily to CBT (Goldapple et al., 2004; Fu et al., 2008), a treatment which modifies attentional biases and reinforces cognitive emotion-regulation strategies. Rostral ACC and inferior parietal cortex together participate in task switching (Liston et al., 2006), the resolution of conflict (Jin et al., 2010), and cognitive control in the form of reappraisal and distraction in the presence of negative affect (McRae).

The dorsal posterior cingulate, an area with a "transitional" pattern of anatomical connectivity linking frontal DMN regions with parietal areas (Vincent et al., 2006; Margulies et al., 2009), balances the internal and external attentional focus in order to retrieve and update behavioral strategies that are not part of the current cognitive set (Pearson et al., 2012). Posterior cingulate lesions in humans and rodents result in deficits in tasks requiring implementation of new strategies in multitasking scenarios and changes of cognitive set (Pearson et al., 2012) and executive dysfunctions. Reduced rest-to-task attenuation of the rACC, VMPFC and dorsal PCC (Grimm et al., 2009), as well as diminished FC between rostral anterior and posterior cingulate in ADHD (Marchetti et al., 2012; Castellanos et al., 2008) support the proposed role of PCC and rostral cingulate communication in attention. 
In the course of MD episodes, FC originating from the rostral cingulate deteriorates further, resulting additionally in diminished communication with the posterior dorsal precuneus, a region with extensive anatomical connectivity with the visual system (Margulies et al., 2009). The function of this area is unclear; however, it has been previously implicated together with the rostral cingulate in task execution and cognitive control difficulties (Halari et al., 2009).

\section{Functional connectivity of the anterior insula in vulnerability and disease}

MD vulnerability is characterized by anticipatory affect biases, including increased threat reactivity (Dearing and Gotlib, 2009). This proclivity of at-risk individuals to interpret anticipated stimuli or situations as more emotional, threatening or generally negative and even more selfrelevant might be due to connectivity dysfunctions such as the one we observed between the anterior insula and the PAG (Grupe and Nitschke, 2013). FC between the anterior insula and the PAG determines whether a stimulus is perceived as painful (Ploner et al., 2010), is higher during selfexperienced versus observed pain (Lamm et al., 2010; Zaki et al., 2007), increases during stressful cognitive tasks (Linnman et al., 2013), and is modulated by the proximity of a virtual predator (Mobbs et al., 2007).

During the MD state, the anterior insula exhibits widespread connectivity abnormalities with regions central in networks that underlie processing of face stimuli (Stuhrmann et al., 2011). These connectivity abnormalities concern enhanced positive FC with the caudate and putamen, the fusiform gyrus and the cerebellum. Fusiform's specialization in face recognition is long known (Kanwisher and Yovel, 2006; lidaka, 2014) while the dorsal striatum has been repeatedly implicated in face processing with a role in reward (Tricomi and Fiez, 2008; Knutson and Greer, 2008; Mattfeld et al., 2011; Pessiglione et al., 2006; Engelmann et al., 2014; Chantiluke et al., 2012; Upadhyay et al., 2010; Kayser et al., 2012), learning (Nomura and Reber, 2008) and emotion processing (Fitzerald et al., 2008; Fu et al., 2008; Stuhrmann et al., 2011; Ajilchi and Nejati, 2013). On the other hand, while the cerebellum is one of the most identified regions in MD (Liu et al., 2012; Baldaçara et al., 2008; Sui et al., 2009), its role in MD abnormalities remains unknown. Anterior insula-cerebellum disrupted communication might be related to self-processing (Buckner et al., 2011, Modinos et al., 2009), intense positive (Blood and Zatorre, 2001) and negative (Peng et al., 2011) feelings and cognitive processing (Dosenback et al., 2007; Chantiluke et al., 2012; Vasic et al., 2008), while a recent transcranial direct stimulation highlighted the role of the cerebellum in faces processing (Ferrucci et al., 2012). It is thus possible that this network of FC disturbances originating from the anterior insula subserves mood congruent biases in processing of face stimuli during MD episodes. 


\section{Functional connectivity alterations in other OMPFC regions}

Our FC results suggest that alterations in pathways linking OMPFC regions and primary visual areas, involved in attention (Seeley et al., 2007), working memory (Zeng et al., 2012; Chantiluke et al., 2012; Chadick et al., 2014) and interpretation of incoming stimuli (Caspers et al., 2011), are present before the onset of MD and get further exacerbated during the acute phase. In MD vulnerability, FC between dorsomedial BA9 and the caudal intraparietal sulcus and between dorsal cingulate d32 and superior occipital cortex is altered. Interestingly, during MD episodes, disturbance of FC with this superior occipital region is shared by the lateral OFC (area 47/12) which shows additionally impaired communication with the inferior temporal cortex. The lateral OFC, via extensive anatomical connections (Barbas, 1988; Morecraft et al., 1992; Carmichael \& Price, 1995; Rolls, 2004), feeds back to ventral visual areas top-down interpretations or predictions about the incoming visual stimuli. This information is postulated to facilitate visual object recognition (Chaumon), the inference of the value association of incoming visual stimuli (Chaumon et al., 2013; Rolls, 2004; Kringelbach and Rolls, 2004; Kringelbach, 2005) and regulate the influence of the emotional information conveyed by incoming sensory inputs (Hooker and Knight, 2006).

Executive dysfunctions in the acute MD phase might be related to aberrant connectivity of orbitofrontal and cingulate areas. As mentioned previously, central OFC (area 11) shows aberrant communication with area $7 \mathrm{~m}$ of the dorsal precuneus during depressive episodes. Both areas appear to be parts of the frontoparietal network (Yeo et al., 2011) and, thus, their aberrant communication is likely to underlie difficulties in executive functions of patients. OFC area 11 is considered an interface of cognition and affect, participating both in the redirection of a response following a violation in stimulus contingencies and in changes of emotional state (Nobre et al., 1999). Precuneus area $7 \mathrm{~m}$ preferentially connects with occipital and parietal areas linked to visual processing and frontal areas associated with motor planning (Buckner et al., 2008). It is activated in the presence of set initiation signals (Fair et al., 2007), during simulations of process versus outcome reward task (Gerlach et al., 2014), in error detection and oddball tasks (Harsay et al., 2014). This part of the precuneus shows increased nodal centrality in first-episode MD (Zhang et al., 2011), decreased activation to attentional targets and differences in deactivation to sad versus neutral distractors (Wang et al., 2008), a finding linked to executive dysfunction in MD.

On the cingulate cortex, anterior cingulate area p24 has abnormally enhanced FC with a region of the anterior PFC, part of the cingulo-opercular network, which has been found to control goal-directed behavior through the stable maintenance of task sets (Dosenbach et al., 2006). Anterior midcingulate area a24' (aMCC) shows increased FC with the DLPFC and the paracentral lobule. The aMCC is activated during a variety of cognitive tasks 
including conflict monitoring (Compton et al., 2003, Sohn et al., 2007; Ursu et al., 2009), error detection (Gehring and Fencsik, 2001; Pourtois et al., 2010), response selection (Awh and Gehring, 1999; Paus, 2001), and attentional control (Crottaz-Herbette and Menon, 2006; Luo et al., 2007). The paracentral lobule has been implicated in conflict and emotional processing (Compton et al., 2003) and has been shown to have FC with the centromedial amygdala, an output region of the amygdala which facilitates motor responding, reward processing and increased attention and cortical readiness (Krain et al., 2009). Gray and white matter abnormalities of the paracentral lobule have been reported for non-refractory MD (Gong et al., 2011; Qiu et al., 2014) as well as increased perfusion (Parkes et al., 2009) and glucose metabolism following vagus nerve stimulation (Pardo et al., 2008).

\section{Limitations}

Our study was the first to dissociate functional connectivity abnormalities in MD vulnerability and disease. Thus replication of our results is needed, particularly given the heterogeneity of the MD population. Our interpretation of the functional role of the FC changes is post-hoc and it remains to be tested in future studies. The connectivity changes in MD vulnerability and disease reported here were found in whole-brain maps with left-hemisphere seeds. Finally, we did not find significant correlations between our FC changes and MD clinical scales. While lack of power might account for this, it is also possible that current syndrome classifications lack specificity and sensitivity and should be replaced by direct measures of the processes sub-served by the functional networks.

\section{Conclusion \& Significance}

Our study demonstrates that data-driven definitions of cortical fields can be used to systematically and in an unbiased way map connectivity alterations of extended cortical regions in psychiatric diseases. We highlight here OMPFC FC markers of MD vulnerability and disease. Our findings might implications for improving diagnostic and therapeutic applications in connectivity syndromes such as MD. 


\section{Supplement}

\section{Design \& Participants}

Thirty-five MD patients meeting DSM-IV-Text Revision criteria for a nonpsychotic major depressive episode (cutoff BDI-II > 20; mean age 38.89; SD 11.63; 71\% female), thirty-six unaffected first-degree relatives of MD sufferers (mean age 34.03; SD 14.57; 72\% female) and thirty-eight healthy controls (mean age 36.21 ; SD $16.43 ; 68 \%$ female) participated in the study after providing informed consent. The three groups did not differ significantly in terms of age $(F(2,106)=.98 ; p=.38)$, gender $(X 2(2)=0.14 ; p=.93)$, or IQ $(F(2,106)=1.53 ; p=.22)$. Nonetheless, to further remove variability associated with these parameters, we included age and gender as covariates in our fMRI analyses (see below). Participants in all three groups were recruited via advertisements in the local press; MD patients were also recruited from the regional institute for outpatient mental healthcare (RIAGG, Maastricht NL). MD patients recruited from the community were screened for axis I disorders with SCID-I (DSM-IV structured clinical interview) (First et al., 2002) and BDI-II by the experimenter (Clinical Psychologist, MSc.) and trained research assistants. Outpatients of the regional institute for mental healthcare were interviewed for axis I disorders with SCID-I at their intake at the center. All aspects of our screening and experimental procedure were approved by the Medical Ethical Committee of the Academic hospital of Maastricht University and conducted in accordance with the University's and Committee's guidelines.

MD patients were included if they met DSM-IV-Text Revision criteria for major depressive disorder as the primary diagnosis and had a BDI-II score of $>20$ (moderate to severe depression) and excluded if they met criteria for Bipolar I or II, substance dependence or were taking benzodiazepines. MD patients with additional axis I diagnoses (except substance dependence and psychotic disorders) or taking other classes of antidepressants were allowed to participate. Table 1 details the diagnostic and medication histories of MD participants. To exclude the possibility that our main results were driven by comorbidity and medication use in our MD sample we filtered out from our analyses voxels significant in associated comparisons (for details see section on MRI analyses).

Participants with familial MD were included if they had a first-degree relative with $M D$, as detailed in a screening questionnaire administered to them. The questionnaire included DSM-IV items for the diagnosis of MD and the differential diagnosis of Bipolar I, II and psychotic features. Participants in this group were excluded if they had ever been personally diagnosed with any axis I disorder and were assessed with the Symptoms-Checklist 90 (SCL-90) (Derogatis and Savitz, 2000) for current psychiatric

symptomatology (exclusion cutoff for males SCL-90 > 116, females SCL-90 > 130; above average population norms). 
Healthy controls were excluded if they had ever been diagnosed with any axis-I disorder and if they had a first or second degree relative with psychiatric history. They were assessed with the SCL-90 for current psychiatric symptomatology and were excluded based on the same cutoffs as the familial MD group. All participants were screened for the following somatic conditions and MRI contra-indications: neurological disorders, epilepsy, severe head injury, claustrophobia, pregnancy or lactation, metal implants, pacemakers and intrauterine contraceptive devices.

\section{Magnetic Resonance Imaging Acquisition}

Scanning was conducted on a Siemens MAGNETOM Allegra 3Tesla MRI head-only scanner. Head motion was constrained by the use of foam padding. Magnetic resonance imaging data, acquired in one scanning session for all participants, included a resting state EPI scan (repetition time $=2.5 \mathrm{sec}, 153$ or 203 volumes) optimized for the reduction of susceptibility artefacts, a high-resolution T1-weighted anatomical scan and a field map. During the resting state scan, participants were instructed to fixate on a cross at the center of the screen, keep their eyes open and refrain from intentionally engaging in specific mental tasks or falling asleep. For each subject, 153 or 203 T2*- weighted gradient echo planar images (EPI) with 41 slices were acquired. EPI can suffer substantial loss of BOLD sensitivity and geometric distortions due to magnetic field inhomogeneities near air tissue interfaces. In order to minimize MRI signal loss and recover the true spatial signal positions in the OFC we: a) used an optimized echo time, b) tilted the slices $\left(\sim 30^{\circ}\right.$ angle), and c) generated a field map to offline correct susceptibility-related signal displacements. Imaging parameters for the resting state sequence were as follows: TR, $2500 \mathrm{~ms}$; TE, $25 \mathrm{~ms}$; flip angle, $90^{\circ}$; matrix size, $128 \times 96$; and FOV, $256 \mathrm{~mm}$; distance factor, $20 \%$; resulting in a voxel size of $2 \times 2 \times 3 \mathrm{~mm}$. The gradient echo image used to generate the field map had the same grid and slice orientation as the functional images (TR, $704 \mathrm{~ms}$; TE, $5.11,7.57 \mathrm{~ms}$; flip angle, $60^{\circ}$ ). In order to enable the localization of functional data, a high-resolution T1-weighted image was acquired with the following parameters: TR, 2250ms; TE, $2.6 \mathrm{~ms}$; flip angle, 9०; FOV, 256mm; slice thickness, $1 \mathrm{~mm}$; matrix size, 256X256; number of slices, 192; voxel size, 1X1X1mm.

\section{Preprocessing}

Preprocessing of fMRI data was performed using SPM 5 software and MATLAB scripts (Mathworks, Natick, MA, USA). The functional data were subjected to the following preprocessing: slice time correction, spatial correction using the field map, realignment, co-registration with the anatomical scan, normalization to the Montreal Neurological Institute (MNI) 152 template, reslicing to $2 \mathrm{~mm}$ isotropic voxels and smoothing with a $6 \mathrm{~mm}$ 
full width half maximum Gaussian kernel. The results of co-registration and normalization step were visually inspected. T1-weighted images were segmented into grey matter, white matter and CSF tissue maps and these maps were used later in the analyses. Further, we removed non-neuronal contributions from the BOLD signal by regressing the following nuisance variables: the six realignment parameters obtained by rigid body head motion correction, the time series extracted from cerebrospinal fluid and white matter, the session specific mean and the intrinsic autocorrelations. The residual volumes of the multiple regression were Fourier band pass filtered $(0.01-0.1 \mathrm{~Hz})$.

\section{Frame-wise Displacement Correction}

Head motion has been shown to significantly distort measures of FC (underestimating long-range and overestimating short-range connectivity) while commonly used preprocessing steps (e.g. realignment of volumes to the first scan, regression of the realignment parameters) do not adequately counter its effect (van Dijk et al., 2012; Power et al., 2012). In order to reduce the bias of head motion in our data we took the following approach: 1) we estimated the scan-to-scan head motion and identified scans during which the frame-wise displacement exceeded a particular threshold (absolute motion difference in the $z$ direction $>0.4 \mathrm{~mm}$ ( $1 / 10$ of voxel size); rotation in the $x$ direction $>0.260$ (angle corresponding to $0.4 \mathrm{~mm} z$-displacement of frontopolar voxels, assuming the rotation point in the middle of the brain is $88 \mathrm{~mm}$ from the anterior end of the brain's frontal pole) (Talairach and Tournoux, 1988), 2) we marked and excluded the identified volumes together with the 1-back and the 2-forward frames (to avoid spin history assumptions' violations caused by movement) (Power et al.), and 3) we included in the analyses only participants for whom at least 120 volumes (i.e. 5 minutes) (van Dijk et al., 2010) of resting data were available after the correction (mean duration, $6.4 \mathrm{~min}$; SD, $0.8 \mathrm{~min}$ ). To ensure that differences in our biomarkers analyses were not better accounted for by the amount of data available after correcting for frame-wise displacements, we quantified the amount of volumes available for FC analyses in each group and performed analysis of variance ( $p>.63)$. To ensure that differences in our main analyses could not be explained by differences in the remaining frame-wise displacement, we compared the post-cleaning mean relative translation distance between the three groups using analysis of variance $(p>.57)$.

\section{OMPFC Cortical map}

Our aim was to parcellate the entire OMFPC into distinct areas based on its resting state FC. FC at rest, reveals a type of "default" functional organization of the brain that while not confined by (as it reflects both direct and indirect connections) nevertheless strongly reflects the underlying structural wiring (Cloutman and Ralph, 2012; Miranda-Dominiquez et al., 
2014). Here we used the intrinsic FC of the OMPFC, i.e. the connectivity of all OMPFC voxels among themselves, to delineate distinct areas with the Louvain modularity-detection algorithm (Blondel et al., 2008). The graph theory metric of modularity is a statistic that quantifies the degree to which a network can be subdivided into groups of nodes with higher than chance connectivity in between them (Girvan and Newman, 2002). Applied in brain networks, it can be used to delineate neurobiologically meaningful functional units, called modules, which perform specialized neural processing (reflecting the balance between functional integration and segregation that characterizes brain organization) (Meunier et al., 2009; Goulas et al., 2012). The first step in this procedure is the construction of a correlation matrix. After preprocessing and reslicing the data $(3 \times 3 \times 3 \mathrm{~mm}$ voxel size), we extracted from the first resting state scan of each participant the time-course of all OMPFC voxels in the left hemisphere using the intersection of the normalized grey matter mask of each individual with an OMPFC ROI mask (thus including only each subject's grey matter voxels). The ROI mask comprised of the following Automated Anatomical Labeling (AAL) map labels: left superior orbital gyrus, left middle orbital gyrus, left inferior orbital gyrus, left medial orbital gyrus, left rectal gyrus and left anterior cingulate gyrus (WFU PickAtlas; Tzourio-Mazoyer et al., 2002; Maldjian et al., 2003; Maldjian et al., 2004). The mask also included the cortex region corresponding to the left frontal superior medial AAL label, extending dorsally until the horizontal border defined by the anterior cingulate AAL label (manually drawn using MRIcron; Rorden and Brett, 2000). We calculated the Pearson's r correlation coefficient for every OMPFC voxel with every other OMPFC voxel using the extracted timecourses and constructed an $\mathrm{N} X \mathrm{~N}$ correlation matrix.

A common step before the application of modularity is to binarize the correlation matrix by applying an absolute weight threshold (Rubinov and Sporns, 2010) such that weak or non-significant links, corresponding most likely to spurious connections, are discarded, while graph connectedness remains intact. Since the threshold value is arbitrarily determined and no sensible "rule-of-thumb" exists, we defined the following range of thresholds for the correlation matrix over which the parcellation algorithm was applied: $0.005,0.01,0.015,0.02,0.025,0.03,0.035,0.04,0.045$ and 0.05 . From these thresholds we chose the one for which modularity was maximum while graph connectedness was not significantly affected.

To partition the binarized correlation matrix into distinct functional subunits we then employed the Louvain module detection algorithm (implemented in MatLab as part of the Brain Connectivity Toolbox; Rubinov and Sporns, 2010), one of the best performing algorithms (Lancichinetti and Fortunato, 2009) for fast and efficient detection of modules in extended networks. For each dataset we run 50 repetitions of the parcellation algorithm and at the end of this process selected the parcellation solution with the highest $Q$. The parcellation procedure results in the unique classification of 
every voxel in the OMPFC into one of the modules in the solution. This classification was transformed into a 3D parcellation module map in order to visualize the distinct modules identified in each subject. All the analyses were performed using MATLAB (Mathworks, Natick, MA, USA).

After partitioning the OMPFC patch into modules in each individual dataset, we integrated the parcellation results at the group level based on the spatial similarity between the modules. Spatial similarity was defined as the inverse of the Euclidean distance between the center of mass (COM) of modules from two different parcellations. The integration progressed iteratively. At each iteration, the parcellation by parcellation cost matrix for matching every individual parcellation with every other individual parcellation was computed. Then, the pairs with the lowest matching cost were merged one after the other by weighted averaging of the COMs of assigned modules. The merged parcellations entered the next iteration level and the procedure was repeated until a final set of COMs was obtained. Matching of pairs of parcellations was accomplished by the modified Hungarian assignment algorithm (Munkres, 1957), and comprised of assigning modules from the two parcellations in a way that minimized the average distance (i.e., the cost) between the COMs of assigned modules.

Averaging of COMs from assigned modules during merging was performed in a weighted manner. Each module of each parcellation started at the first iteration with a weight of 1.0. Whenever, during the merging process, a module remained un-assigned (i.e., due to different numbers of modules in the two parcellations), it was penalized by decreasing its weight. As a consequence, the misfitting module's influence during averaging with another module at the next level was reduced. In the final set of COMs, modules with a weight $<0.5$ were eliminated. As a final COM-distance check, the $10 \%$ modules farthest away from the final group COM were eliminated from each cluster.

At the end of this procedure, each final COM represents a cluster of modules from individual parcellations merged into this common COM. Note that not every initial module is represented in every final cluster, due to different numbers of modules per parcellation and due to the final COMdistance check. Finally, for each cluster a new COM was computed as the average coordinates of all voxels with a positive within-module degree zscore (Guimerá and Amaral, 2005) (i.e. only voxels/nodes tightly connected within their module had an influence on the clusters' COMs).

\section{fMRI FC Biomarkers Analysis}

To systematically examine connectivity changes in each OMPFC cortical field associated with MD, we generated whole-brain FC maps seeding from the COM (Table X) of each of the 19 subregions. The timecourse of each seed was de-noised (Eigen decomposition) before calculating Pearson's $r$ between each seed's time-course and the time-course of all 
voxels in the brain. Values in the whole-brain FC maps were $r$ - to- $Z$ transformed using Fisher's formula (REFERENCE). Subsequently, the FC maps of OMPFC field were subjected to an analysis of covariance (ANCOVA) with group as a factor and age, gender and IQ Raven score as covariates.

To test for connectivity biomarkers of MD vulnerability, we created conjunction maps of the F contrasts between 1) the healthy controls and the MD patients and 2) the healthy controls and the familial MD participants. The $F$ contrasts entered in SPM for the conjunction maps in this analysis were: 1) Healthy controls $\sim$ MD patients $(1,0,-1)$ and 2 ) Healthy controls $\sim$ familial $\mathrm{MD}(0,1,-1)$. A third $\mathrm{F}$ contrast was created for: MD patients $\sim$ familial MD $(1,-1,0)$. Thus, MD vulnerability FC biomarkers were defined as changes: 1$)$ significantly different between the healthy controls and the MD patients and vulnerable individuals (conjunction map) and logical 2) not significantly different between MD patients and MD vulnerable individuals (third $F$ map). Conjunction maps were tested for significance with Monte Carlo simulations (10.000 iterations; voxel-level $p=.01$, cluster-level $p=.0025$ - Bonferroniadjusted for 19 independent comparisons). To retain in the analysis only brain areas with altered $\mathrm{FC}$ in both the disease and the vulnerability state, we masked out of the conjunction maps all voxels significant at a lenient threshold (.001 uncorrected) in the F contrast between MD patients and MD vulnerable participants.

To test for connectivity biomarkers of MD disease, we created conjunction maps of the F contrasts between 1) the MD patients and the healthy controls and 2) the MD patients and the MD vulnerable individuals. The F contrasts entered in SPM for the conjunction map in this analysis were: 1) MD patients healthy controls $(1,0,-1)$ and 2$)$ MD patients familial $M D(1,-1,0)$. A third $F$ contrast was created for: Healthy controls familial $\operatorname{MDD}(0,1,-1)$. Thus, MD disease FC biomarkers were defined as changes: 1) significantly different between the MD patients and the familial MD participants and healthy controls (conjunction map) and logical 2) not significantly different between familial MD and healthy controls (third F map). Conjunction maps were tested for significance with Monte Carlo simulations (10.000 iterations; voxel-level $p=.01$, cluster-level $p=.0025$ - Bonferroniadjusted for 19 independent comparisons). To retain in the analysis only brain areas with altered FC in exclusively the disease state, we masked out from the conjunction maps all voxels significant at a lenient threshold (.001 uncorrected) in the F contrast between familial MD and controls.

Since ANOVA is a general test for differences between groups, we determined the direction of FC differences in MD vulnerability and disease with ROI-based post-hoc tests in SPSS using the r-Z-transformed connectivity values of the statistically significant voxels in the conjunction maps. After being cluster-based corrected (Monte Carlo) the 19 FC conjunction maps (one for each OMPFC subregion) were used as binary 
masks to extract the $Z$ values of each individual in the three groups.

Subsequently, analyses of variance and post-tests with Bonferroni adjusted $p$ values were run in SPSS to establish the direction of the FC changes.

Finally, to relate our FC biomarkers to disease variables, correlation analyses for the MD group between the disease biomarkers and the clinical measures were run in SPSS.

\section{Control Analysis}

To ensure that the reported connectivity differences were not driven by antidepressant medication use and comorbidity in our MD sample, we filtered out of the F maps voxels significant at a lenient threshold (.001 uncorrected) in the comparison between MD medication and comorbidity subgroups. ANOVA tests concerned two MD-patient subgroups (one subgroup using one or more antidepressants and one not using antidepressants at all; one subgroup having at least an additional axis I diagnosis and one having only an MD diagnosis) for medication use and comorbidity (see Supplementary Table 1 for details). To establish that our FC differences were not accounted for by probability of grey matter differences in the tested voxels we removed from our $F$ maps all voxels significant in an ANOVA between all groups at a lenient threshold (.001 uncorrected). For this comparison we used the probability of the significant FC voxels/clusters being grey matter of the modulated and warped grey matter tissue masks created during the segmentation step of the preprocessing. 


\section{Supplementary Figures \& Tables}

Supplementary Table 1. Demographic and clinical data of the MD patient group.

\begin{tabular}{|c|c|c|c|c|}
\hline Age & Gender & Axis I Comorbidity & Current Medications & Med Type \\
\hline 22 & $\mathrm{~F}$ & & & \\
\hline 31 & $\mathrm{~F}$ & PTSD & Venlafaxine & SNRI \\
\hline 59 & $\mathrm{~F}$ & & & \\
\hline 49 & M & Social Phobia & & \\
\hline 34 & $\mathrm{~F}$ & & & \\
\hline 18 & $\mathrm{~F}$ & & Citalopram & SSRI \\
\hline 25 & $\mathrm{~F}$ & Social Phobia & Sertraline & SSRI \\
\hline 49 & $\mathrm{~F}$ & & Paroxetine & SSRI \\
\hline 20 & M & PTSD & & \\
\hline 52 & M & & & \\
\hline 31 & $\mathrm{~F}$ & & Nortriptyline & TCA \\
\hline 23 & $\mathrm{~F}$ & Specific Phobia & Bupropion & NDRI \\
\hline 52 & $\mathrm{~F}$ & Specific Phobia & Citalopram & SSRI \\
\hline 47 & M & & Venlafaxine, Seroquel & $\begin{array}{l}\text { SNRI, } \\
\text { Antipsychotic }\end{array}$ \\
\hline 44 & M & & Citalopram, Mirtazapine & $\begin{array}{l}\text { SSRI, } \\
\text { TetraCA }\end{array}$ \\
\hline 25 & $\mathrm{~F}$ & & Agomelatine & Melatonergic \\
\hline 36 & $\mathrm{~F}$ & & & \\
\hline 39 & $\mathrm{~F}$ & Social Phobia, GAD & & \\
\hline 52 & M & & & \\
\hline 44 & M & GAD & Paroxetine & SSRI \\
\hline 42 & $\mathrm{~F}$ & Panic Disorder with agoraphobia & Citalopram & SSRI \\
\hline 54 & $\mathrm{~F}$ & & & \\
\hline 55 & $\mathrm{~F}$ & PTSD & Citalopram & SSRI \\
\hline 49 & $\mathrm{~F}$ & & & \\
\hline 37 & M & Specific Phobia, Social Phobia & & \\
\hline 38 & $\mathrm{~F}$ & Social Phobia & & \\
\hline 44 & $\mathrm{~F}$ & & & \\
\hline 36 & $\mathrm{~F}$ & Social Phobia & & \\
\hline 38 & $\mathrm{~F}$ & & Escitalopram & SSRI \\
\hline 22 & $\mathrm{~F}$ & & & \\
\hline 19 & $\mathrm{~F}$ & $\begin{array}{l}\text { GAD, Panic with agoraphobia, } \\
\text { ADHD/ADD }\end{array}$ & Citalopram & SSRI \\
\hline 34 & M & $\begin{array}{l}\text { Panic Disorder with agoraphobia, } \\
\text { Social Phobia }\end{array}$ & Escitalopram & SSRI \\
\hline 43 & M & Social Phobia & & \\
\hline 54 & $\mathrm{~F}$ & Agoraphobia & & \\
\hline 44 & $\mathrm{~F}$ & Panic disorder, OCD & Fluvoxamine & SSRI \\
\hline
\end{tabular}


Abbreviations: PTSD (Post Traumatic Stress Disorder); GAD (Generalized Anxiety Disorder); ADHD/ADD (Attention Deficit Hyperactivity Disorder); OCD (Obsessive Compulsive Disorder); SNRI (Serotonin Norepinephrine Reuptake Inhibitor); SSRI (Selective Serotonin Reuptake Inhibitor); TCA (Tricyclic Antidepressant); NDRI (Norepinephrine Dopamine Reuptake Inhibitor); TetraCA (Tetracyclic Antidepressant).

Supplementary Table 2. Summary table of MRI results

\begin{tabular}{|c|c|c|c|c|c|c|}
\hline Map & OMPFC area & Anatomy & Average MNI & p Value & $\begin{array}{l}\text { Post-Hoc } \\
\text { p(Bonf.) }\end{array}$ & $\begin{array}{c}\text { Post-Hoc } \\
\text { p(Bonf.) }\end{array}$ \\
\hline MD Vulnerability & Ant. Insula & Brainstem, PAG & $3,-26,-15$ & 0.000005 & $0.000014^{a}$ & $0.000237^{b}$ \\
\hline MD Vulnerability & p32 & Dorsal PCC & $1,-30,30$ & 0.000048 & $0.000091^{a}$ & $0.001494^{b}$ \\
\hline MD Vulnerability & p32 & BA $40 / 39$ & $44,-50,45$ & 0.000027 & $0.000116^{a}$ & $0.000363^{b}$ \\
\hline MD Vulnerability & BA9 & IPS & $-30,-72,32$ & 0.000001 & $0.000026^{a}$ & $0.000030^{\mathrm{b}}$ \\
\hline MD Vulnerability & d32 & Occipital & $23,-91,20$ & 0.000001 & $0.000004^{a}$ & $0.000030^{\mathrm{b}}$ \\
\hline MD Disease & $47 / 12$ (OFC) & IT & $48,-46,-18$ & 0.000001 & $0.000025^{c}$ & $0.000004^{\mathrm{d}}$ \\
\hline MD Disease & 47/12 (OFC) & Occipital (RH) & $26,-92,20$ & 0.000003 & $0.000061^{c}$ & $0.000016^{\mathrm{c}}$ \\
\hline MD Disease & 47/12 (OFC) & Occipital (LH) & $-25,-87,21$ & 0.000001 & $0.000011^{c}$ & $0.000007^{\circ}$ \\
\hline MD Disease & 47/12 (OFC) & $\begin{array}{l}\text { Paracentral } \\
\text { Lobule }\end{array}$ & $2,-47,73$ & 0.000003 & $0.000046^{c}$ & $0.000013^{\mathrm{c}}$ \\
\hline MD Disease & Ant. Insula & Cerebellum & $29,-61,-36$ & 0.000001 & $0.000007^{c}$ & $0.000014^{\mathrm{c}}$ \\
\hline MD Disease & Ant. Insula & Fusiform & $34,-72,-18$ & 0.000006 & $0.000381^{c}$ & $0.000010^{\circ}$ \\
\hline MD Disease & Ant. Insula & Putamen & $-23,5,5$ & 0.000002 & $0.000040^{c}$ & $0.000008^{\circ}$ \\
\hline MD Disease & Ant. Insula & Caudate & $13,25,2$ & 0.000538 & $0.000984^{c}$ & $0.000514^{\mathrm{c}}$ \\
\hline MD Disease & p32 & Precuneus (LH) & $-8,-72,33$ & 0.000000 & $0.000000^{c}$ & $0.000036^{\circ}$ \\
\hline MD Disease & p32 & Precuneus (RH) & $11,-71,41$ & 0.000130 & $0.000352^{c}$ & $0.001185^{\circ}$ \\
\hline MD Disease & p24 & aPFC & $-27,58,15$ & 0.000269 & $0.000082^{c}$ & $0.000097^{\circ}$ \\
\hline MD Disease & a24' & $\begin{array}{l}\text { DLPFC } \\
\text { Paracentral }\end{array}$ & $-10,42,47$ & 0.000037 & $0.000168^{c}$ & $0.000290^{\circ}$ \\
\hline MD Disease & $a 24^{\prime}$ & Lobule & $-7,-25,63$ & 0.000008 & $0.000124^{c}$ & $0.000034^{d}$ \\
\hline MD Disease & 11 (OFC) & Precuneus & $4,-66,52$ & 0.000929 & $0.004654^{c}$ & $0.002322^{\circ}$ \\
\hline
\end{tabular}

Post-hoc Bonferroni corrected $p$ values a and $b$ concern the comparisons MD patient group versus $H C$ and familial $M D$ group versus $H C$ respectively for the $M D$ vulnerability markers. Post-hoc Bonferroni corrected $p$ values $c$ and $d$ concern the comparisons MD patient group versus the familial MD group and MD patient group versus $H C$ respectively for the $M D$ disease markers. 


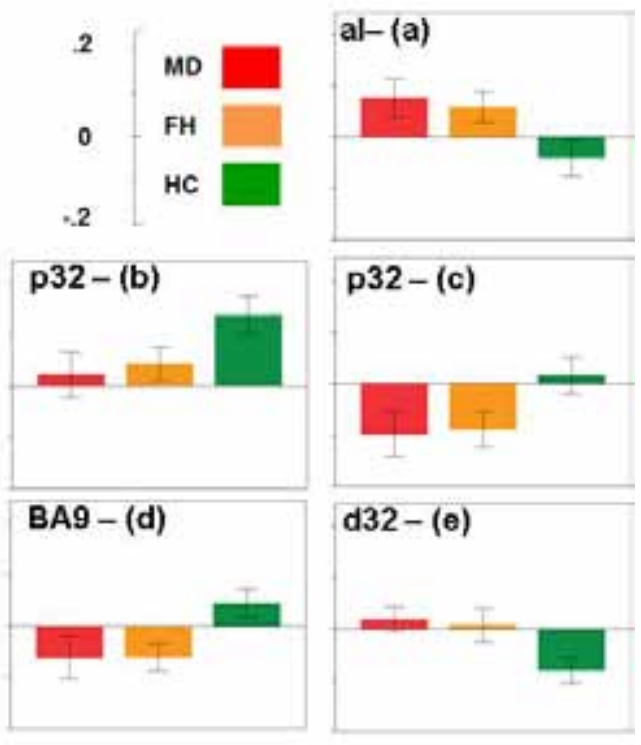

Supplementary Figure 1. Bar plots for FC differences in MD vulnerability (Error Bars $=95 \%$ Confidence Intervals). Region nomenclature and letters correspond to main Figure 1.

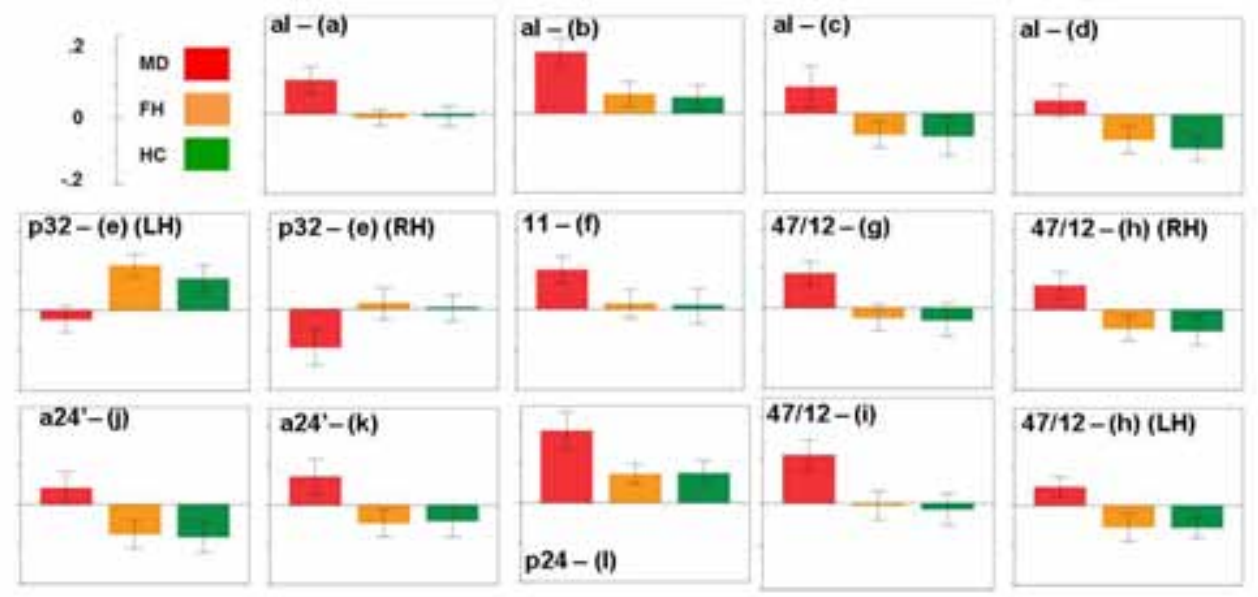

Supplementary Figure 2. Bar plots for FC differences in MD vulnerability (Error Bars $=95 \%$ Confidence Intervals). Region nomenclature and letters correspond to main Figure 2. 
Supplementary Table 3. Mean and standard deviations of functional connectivity per group.

MD FH HC

\begin{tabular}{|c|c|c|c|c|c|c|c|c|}
\hline Map & OMPFC area & Anatomy & Mean & SD & Mean & SD & Mean & SD \\
\hline $\begin{array}{l}\text { MD } \\
\text { Vulnerability }\end{array}$ & Ant. Insula & Brainstem, PAG & 0.08 & 0.11 & 0.06 & 0.09 & -0.04 & 0.11 \\
\hline $\begin{array}{l}\text { MD } \\
\text { Vulnerability }\end{array}$ & p32 & $\mathrm{dPCC}$ & 0.02 & 0.13 & 0.04 & 0.09 & 0.14 & 0.12 \\
\hline $\begin{array}{l}\text { MD } \\
\text { Vulnerability }\end{array}$ & p32 & BA $40 / 39$ & -0.10 & 0.13 & -0.09 & 0.10 & 0.02 & 0.11 \\
\hline $\begin{array}{l}\text { MD } \\
\text { Vulnerability }\end{array}$ & BA9 & IPS & -0.06 & 0.12 & -0.06 & 0.08 & 0.04 & 0.08 \\
\hline $\begin{array}{l}\text { MD } \\
\text { Vulnerability }\end{array}$ & d32 & Occipital & 0.02 & 0.07 & 0.01 & 0.10 & -0.08 & 0.08 \\
\hline MD Disease & 47/12 (OFC) & IT & 0.11 & 0.10 & -0.01 & 0.10 & -0.01 & 0.11 \\
\hline MD Disease & 47/12 (OFC) & Occipital (RH) & 0.06 & 0.10 & -0.04 & 0.09 & -0.05 & 0.11 \\
\hline MD Disease & 47/12 (OFC) & Occipital (LH) & 0.05 & 0.07 & -0.05 & 0.10 & -0.06 & 0.08 \\
\hline MD Disease & 47/12 (OFC) & $\begin{array}{l}\text { Paracentral } \\
\text { Lobule }\end{array}$ & 0.09 & 0.09 & -0.02 & 0.09 & -0.03 & 0.12 \\
\hline MD Disease & Ant. Insula & Cerebellum & 0.08 & 0.10 & -0.01 & 0.06 & -0.01 & 0.08 \\
\hline MD Disease & Ant. Insula & Fusiform & 0.04 & 0.12 & -0.06 & 0.10 & -0.09 & 0.09 \\
\hline MD Disease & Ant. Insula & Putamen & 0.15 & 0.11 & 0.05 & 0.09 & 0.04 & 0.09 \\
\hline MD Disease & Ant. Insula & Caudate & 0.07 & 0.16 & -0.05 & 0.10 & -0.06 & 0.15 \\
\hline MD Disease & p32 & Precuneus (LH) & -0.02 & 0.09 & 0.11 & 0.08 & 0.08 & 0.10 \\
\hline MD Disease & p32 & Precuneus (RH) & -0.09 & 0.13 & 0.02 & 0.11 & 0.00 & 0.10 \\
\hline MD Disease & $\mathrm{p} 24$ & aPFC & 0.18 & 0.14 & 0.07 & 0.08 & 0.07 & 0.10 \\
\hline MD Disease & a24' & DLPFC & 0.07 & 0.13 & -0.05 & 0.10 & -0.04 & 0.12 \\
\hline MD Disease & a24' & $\begin{array}{l}\text { Paracentral } \\
\text { Lobule }\end{array}$ & 0.04 & 0.12 & -0.07 & 0.11 & -0.08 & 0.12 \\
\hline MD Disease & 11 (OFC) & Precuneus & 0.10 & 0.10 & 0.01 & 0.10 & 0.01 & 0.13 \\
\hline
\end{tabular}




\section{References}

Ajilchi B, Nejati V. 2013. Attention Bias to Sad Faces and Images: Which Is Better for Predicting Depression? Open J Depress. 02:19-23.

American, Psychiatric Association (1994): Diagnostic and Statistical Manual of Mental Disorders, 4th ed. Washington DC: American Psychiatric Press.

Awh E, Gehring WJ. 1999. The anterior cingulate cortex lends a hand in response selection. Nat. Neurosci. 2:853-854.

Baldaçara L, Guilherme J, Borgio F, Luiz A, Lacerda TD, Jackowski AP. 2008. Cerebellum and psychiatric disorders $\mathrm{O}$ cerebelo e os transtornos psiquiátricos. Sinapse. 30: 281-289.

Barbas H.1988. Anatomic organization of basoventral and mediodorsal visual recipient prefrontal regions in the rhesus monkey. Journal of Comparative Neurology. 276:313342.

Barbas H, Pandya DN.1989. Architecture and intrinsic connections of the prefrontal cortex in the rhesus monkey. J Comp Neurol. 286:353-375.

Barnes KA, Cohen AL, Power JD, Nelson SM, Dosenbach YBL, Miezin FM, et al. 2010). Identifying Basal Ganglia divisions in individuals using resting-state functional connectivity MRI. Front Syst Neurosci. 4: 18.

Beck AT, Steer RA, Ball R, Ranieri W. 1996. Comparison of Beck Depression Inventories-IA and - II in psychiatric outpatients. J Pers Assess. 67:588-597.

Boes AD, McCormick LM, Coryell WH, Nopoulos P. 2008. Rostral Anterior Cingulate Cortex Volume Correlates with Depressed Mood in Normal Healthy Children. Biol Psychiatry. 63: 391-397.

Blondel VD, Guillaume JL, Lefebvre E. 2008. Fast unfolding of communities in large networks. J Stat Mech P.10008.

Blood AJ, Zatorre RJ. 2001. Intensely pleasurable responses to music correlate with activity in brain regions implicated in reward and emotion. Proc Natl Acad Sci U S A. 98:1181823.

Browning M, Holmes EA, Charles M, Cowen PJ, Harmer CJ. 2012. Using attentional bias modification as a cognitive vaccine against depression. Biol Psychiatry. 72(7):572-9.

Buckner RL, Andrews-Hanna JR, Schacter DL. 2008. The brain's default network: anatomy, function, and relevance to disease. Ann N Y Acad Sci. 1124: 1-38.

Buckner RL, Krienen FM, Castellanos A, Diaz JC, Yeo BTT. 2011. The organization of the human cerebellum estimated by intrinsic functional connectivity. J Neurophysiol. 106: 2322-45.

Bush G, Luu P, Posner MI. 2000. Cognitive and emotional influences in anterior cingulate cortex. Trends Cogn Sci. 4: 215-222.

Cannon DM, Ichise M, Rollis D, Klaver JM, Gandhi SK, Charney DS, et al. 2007. Elevated serotonin transporter binding in major depressive disorder assessed using positron 
emission tomography and [11C]DASB; comparison with bipolar disorder. Biol Psychiatry. 62: 870-7.

Carmichael ST, Price JL. 1995. Sensory and premotor connections of the orbital and medial prefrontal cortex of macaque monkeys. Journal of Comparative Neurology. 363:642664.

Caspers S, Eickhoff SB, Rick T, von Kapri A, Kuhlen T, Huang R, et al. 2011. Probabilistic fibre tract analysis of cytoarchitectonically defined human inferior parietal lobule areas reveals similarities to macaques. Neuroimage. 58: 362-80.

Caspers S, Schleicher A, Bacha-Trams M, Palomero-Gallagher N, Amunts K, Zilles K .2013. Organization of the human inferior parietal lobule based on receptor architectonics. Cereb Cortex. 23:615-28.

Castellanos FX, Margulies DS, Kelly C, Uddin LQ, Ghaffari M, Kirsch A, et al. 2008. Cingulateprecuneus interactions: a new locus of dysfunction in adult attention-deficit/hyperactivity disorder. Biol Psychiatry. 63:332-7.

Chadick JZ, Zanto TP, Gazzaley A. 2014. Structural and functional differences in medial prefrontal cortex underlie distractibility and suppression deficits in ageing. Nat Commun. 5:4223.

Chantiluke K, Halari R, Simic M, Pariante CM, Papadopoulos A, Giampietro V, Rubia K. 2012. Fronto-striato-cerebellar dysregulation in adolescents with depression during motivated attention. Biol Psychiatry. 71:59-67.

Chaumon M, Kveraga K, Barrett LF, Bar M. 2013. Visual Predictions in the Orbitofrontal Cortex Rely on Associative Content. Cereb Cortex. 2899-2907.

Cloutman LL, Lambon Ralph MA. 2012. Connectivity-based structural and functional parcellation of the human cortex using diffusion imaging and tractography. Front Neuroanat. 6:34.

Compton RJ, Banich MT, Mohanty A, Milham MP, Herrington J, Miller GA, et al. 2003. Paying attention to emotion: an fMRI investigation of cognitive and emotional stroop tasks. Cogn Affect Behav Neurosci. 3: 81-96.

Critchley HD. 2005. Neural mechanisms of autonomic, affective, and cognitive integration. J Comp Neurol. 493: 154-66.

Crottaz-Herbette S, Menon V. 2006. Where and when the anterior cingulate cortex modulates attentional response: combined fMRI and ERP evidence. J. Cogn. Neurosci. 18:766780.

Dastjerdi M, Foster BL, Nasrullah S, Rauschecker AM, Dougherty RF, Townsend JD, et al. 2011.Differential electrophysiological response during rest, self-referential, and nonself-referential tasks in human posteromedial cortex. Proc Natl Acad Sci U S A. 108:3023-8.

Dearing KF, Gotlib IH. 2009. Interpretation of ambiguous information in girls at risk for depression. J Abnorm Child Psychol. 37:79-91.

Derogatis L, Melisaratos N. 1983. The Brief Symptom Inventory: An introductory report. 
Psychological Medicine. 13:595-605.

Derogatis LR, Savitz KL. 2000. The SCL-90-R and the Brief Symptom Inventory (BSI) in Primary Care In: M.E.Maruish, ed. Handbook of psychological assessment in primary care settings, Volume 236 Mahwah, NJ: Lawrence Erlbaum Associates, pp 297-334

Dosenbach NUF, Visscher KM, Palmer ED, Miezin FM, Wenger KK, Kang HC, et al. 2006. A Core System for the Implementation of Task Sets. Neuron. 50: 799-812.

Dosenbach NUF, Fair DA, Miezin FM, Cohen AL, Wenger KK, Dosenbach RA, et al. 2007. Distinct brain networks for adaptive and stable task control in humans. Proc Natl Acad Sci U S A. 104:11073-8.

Downar J, Daskalakis ZJ. 2013. New targets for rTMS in depression: a review of convergent evidence. Brain Stimul. 6:231-40.

Egner T, Etkin A, Gale S, Hirsch J. 2008. Dissociable neural systems resolve conflict from emotional versus nonemotional distracters. Cereb Cortex. 18:1475-84.

Engelmann JB, Berns GS, Dunlop BW. 2014. Depression distorts neural loss aversion in anterior insula and caudate nucleus.78292.

Etkin A, Egner T, Peraza DM, Kandel ER, Hirsch J. 2006. Resolving emotional conflict: a role for the rostral anterior cingulate cortex in modulating activity in the amygdala. Neuron. 51: 871-82.

Fair DA, Dosenbach NUF, Church JA, Cohen AL, Brahmbhatt S, Miezin FM, et al. 2007. Development of distinct control networks through segregation and integration. Proc Natl Acad Sci U S A. 104:13507-12.

Ferrucci R, Giannicola G, Rosa M, Fumagalli M, Boggio PS, Hallett M, et al. 2012. Cerebellum and processing of negative facial emotions: cerebellar transcranial DC stimulation specifically enhances the emotional recognition of facial anger and sadness. Cogn Emot. 26:786-99.

First MB, Spitzer RL, Gibbon M, Williams JBW. 2002. Structured Clinical Interview for DSM-IVTR Axis I Disorders, Research Version, Patient Edition. (SCID-I/P) New York: Biometrics Research, New York State Psychiatric Institute, November 2002.

Fitzgerald PB, Laird AR, Maller J, Daskalakis ZJ. 2008. A meta-analytic study of changes in brain activation in depression. Hum Brain Mapp. 29: 683-95.

Foland-Ross LC, Gotlib IH. 2012. Cognitive and neural aspects of information processing in major depressive disorder: an integrative perspective. Front Psychol. 3:489.

Fortunato S. (2010). Community detection in graphs. Phys Rep. 486:75-174.

Fu CHY, Williams SCR, Cleare AJ, Scott J, Mitterschiffthaler MT, Walsh ND, et al. 2008. Neural responses to sad facial expressions in major depression following cognitive behavioral therapy. Biol Psychiatry 64: 505-12.

Fu CHY, Steiner H, Costafreda SG. 2013. Predictive neural biomarkers of clinical response in depression: a meta-analysis of functional and structural neuroimaging studies of pharmacological and psychological therapies. Neurobiology of Disease. 52:75-83. 
Gehring WJ, Fencsik DE. 2001. Functions of the medial frontal cortex in the processing of conflict and errors. J. Neurosci. 21:9430-9437.

Gerlach KDK, Spreng RN, Madore KP, Schacter DL. 2014. Default network activity couples with frontoparietal control network and reward-processing regions during process and outcome simulations. Soc Cogn Affect Neurosci.1942-1951.

Girvan M, Newman MEJ. 2002. Community structure in social and biological networks. Proc Natl Acad Sci U S A. 99:7821-6.

Goldapple K, Segal Z, Garson C, Lau M, Bieling P, Kennedy S, Mayberg H. 2004. Modulation of Cortical-Limbic Pathways in Major Depression. Arch Gen Psych. 61(1):34-41.

Gong Q, Wu Q, Scarpazza C, Lui S, Jia Z, Marquand A, et al. 2011. Prognostic prediction of therapeutic response in depression using high-field MR imaging. Neuroimage. 55:1497-503.

Goulas A, Uylings HBM, Stiers P. 2012. Unravelling the intrinsic functional organization of the human lateral frontal cortex: a parcellation scheme based on resting state fMRI. J Neurosci. 32:10238-52.

Greicius MD, Flores BH, Menon V, Glover GH, Solvason HB, Kenna H, et al. 2007. Restingstate functional connectivity in major depression: abnormally increased contributions from subgenual cingulate cortex and thalamus. Biol Psychiatry. 62:429-37.

Grimm S, Boesiger P, Beck J, Schuepbach D, Bermpohl F, Walter M, et al. 2009. Altered negative BOLD responses in the default-mode network during emotion processing in depressed subjects. Neuropsychopharmacology. 34:932-843.

Grupe DW, Nitschke JB. 2013. Uncertainty and anticipation in anxiety: an integrated neurobiological and psychological perspective. Nat Rev Neurosci. 14:488-501.

Guimerá R, Amaral LAN. 2005. Functional cartography of complex metabolic networks. Nature. 433:895-900.

Guo H, Cheng C, Cao X, Xiang J, Chen J, Zhang K. 2014. Resting-state functional connectivity abnormalities in first-onset unmedicated depression. Neural Regen Res. 9:153-63.

Halari R, Simic M, Pariante CM, Papadopoulos A, Cleare A, Brammer M, et al. 2009. Reduced activation in lateral prefrontal cortex and anterior cingulate during attention and cognitive control functions in medication-naïve adolescents with depression compared to controls. J Child Psychol Psychiatry. 50:307-16.

Hamani C, Mayberg H, Stone S, Laxton A, Haber S, Lozano AM. 2010. The Subcallosal Cingulate Gyrus in the Context of Major Depression. Biological Psychiatry. 69:301-308.

Harsay HA, Spaan M, Wijnen JG, Ridderinkhof KR. 2012. Error awareness and salience processing in the oddball task: shared neural mechanisms. Front Hum Neurosci. 6:246.

Herwig U, Kaffenberger T, Baumgartner T, Jäncke L (2007): Neural correlates of a "pessimistic" attitude when anticipating events of unknown emotional valence. Neuroimage 34: 848-58. 
Hooker Cl, Knight RT. 2006. The role of lateral orbitofrontal cortex in inhibitory control of emotion. In: Zald DH, Rauch SL, editors. The Orbitofrontal Cortex. Oxford University Press; pp. 307-324.

lidaka T. 2014. Role of the fusiform gyrus and superior temporal sulcus in face perception and ecognition: An empirical review. Jpn Psychol Res. 56:33-45.

Jacobs RH, Jenkins LM, Gabriel LB, Barba A, Ryan K a, Weisenbach SL, et al. 2014. Increased coupling of intrinsic networks in remitted depressed youth predicts rumination and cognitive control. PLoS One. 9:e104366.

Jin Y, Olk B, Hilgetag CC. 2010. Contributions of human parietal and frontal cortices to attentional control during conflict resolution: a 1-Hz offline rTMS study. Exp brain Res. 205:131-8.

Kanwisher N, Yovel G. 2006. The fusiform face area: a cortical region specialized for the perception of faces. Phil. Trans. R. Soc. B. 361:2109-2128.

Kayser AS, Allen DC, Navarro-Cebrian A, Mitchell JM, Fields HL. 2012. Dopamine, corticostriatal connectivity, and intertemporal choice. J Neurosci. 32:9402-9.

Kerr N, Scott J, Phillips ML. 2005. Patterns of attentional deficits and emotional bias in bipolar and major depressive disorder. British Journal of Clinical Psychology. 44: 343-356.

Kim C, Chung C, Kim J. 2010. Multiple cognitive control mechanisms associated with the nature of conflict. Neurosci Lett. 476:156-160.

Knutson B, Greer SM. 2008. Anticipatory affect: neural correlates and consequences for choice. Philos Trans R Soc Lond B Biol Sci. 363:3771-86.

Krain A, Shehzad Z, Margulies DS, Kelly AMC, Uddin LQ, Gotimer K, Biswal BB, Castellanos FX, Milham MP. 2009. Neurolmage Functional connectivity of the human amygdala using resting state fMRI. Neuroimage. 45:614-626.

Kringelbach ML. 2005. The human orbitofrontal cortex: linking reward to hedonic experience. Nat Rev Neurosci. 6:691-702.

Kringelbach ML, Rolls ET. 2004. The functional neuroanatomy of the human orbitofrontal cortex: evidence from neuroimaging and neuropsychology. Prog Neurobiol 72: 341-72.

Lamm C, Meltzoff AN, Decety J. 2010. How do we empathize with someone who is not like us? A functional magnetic resonance imaging study. J Cogn Neurosci. 22:362-376.

Lancichinetti A, Fortunato S. 2009. Community detection algorithms: a comparative analysis. Phys Rev E. 80:056117.

Li B, Liu L, Friston KJ, Shen H, Wang L, Zeng LL, Hu D. 2013. A treatment-resistant default mode subnetwork in major depression. Biol Psychiatry. 74:48-54.

Linnman C, Moulton EA, Barmettler G, Becerra L, Borsook D. 2013. Neuroimaging of the Periaqueductal Gray: State of the Field. Neuroimage. 60(1): 505-522. 
Liston C, Matalon S, Hare TA, Davidson MC, Casey BJ. 2006. Anterior cingulate and posterior parietal cortices are sensitive to dissociable forms of conflict in a task-switching paradigm. Neuron. 50: 643-53.

Liston C, Chen AC, Zebley BD, Drysdale AT, Gordon R, Leuchter B, et al. 2014. Default Mode Network Mechanisms of Transcranial Magnetic Stimulation in Depression. Biol Psychiatry. 76: 17-526.

Liu L, Zeng L-L, Li Y, Ma Q, Li B, Shen H, Hu D. 2012. Altered cerebellar functional connectivity with intrinsic connectivity networks in adults with major depressive disorder. PLoS One. 7:e39516.

Luo, Q., Mitchell, D., Jones, M., Mondillo, K., Vythilingam, M., Blair, R.J., 2007. Common regions of dorsal anterior cingulate and prefrontal-parietal cortices provide attentional control of distracters varying in emotionality and visibility. Neuroimage. 38:631-639.

Maldjian JA, Laurienti PJ, Kraft RA, Burdette JH. 2003. An automated method for neuroanatomic and cytoarchitectonic atlas-based interrogation of fMRI data sets. Neuroimage. 19:1233-1239.

Maldjian JA, Laurienti PJ, Burdette JH. 2004. Precentral gyrus discrepancy in electronic versions of the Talairach atlas. Neuroimage. 21:450-455.

Manoliu A, Meng C, Brandl F, Doll A, Tahmasian M, Scherr M, et al. 2013. Insular dysfunction within the salience network is associated with severity of symptoms and aberrant internetwork connectivity in major depressive disorder. Front Hum Neurosci. 7:930.

Marchetti I, Koster EHW, Sonuga-Barke EJ, De Raedt R. 2012. The default mode network and recurrent depression: a neurobiological model of cognitive risk factors. Neuropsychol Rev. 22:229-51.

Margulies DS, Vincent JL, Kelly C, Lohmann G, Uddin LQ, Biswal BB, et al. 2009. Precuneus shares intrinsic functional architecture in humans and monkeys. Proc Natl Acad Sci U S A.106: 20069-74.

Mars RB, Jbabdi S, Sallet J, O'Reilly JX, Croxson PL, Olivier E, et al. 2011. Diffusion-weighted imaging tractography-based parcellation of the human parietal cortex and comparison with human and macaque resting-state functional connectivity. J Neurosci. 31:4087100.

Mattfeld AT, Gluck M a, Stark CEL. 2011.Functional specialization within the striatum along both the dorsal/ventral and anterior/posterior axes during associative learning via reward and punishment. Learn Mem. 18:703-11.

Mayberg H. 2003. Modulating dysfunctional limbic-cortical circuits in depression: towards development of brain-based algorithms for diagnosis and. Br Med Bull.193-207.

Mcrae K, Hughes B, Chopra S, Gabrieli JDE, Gross JJ, Ochsner KN. 2009. The neural basis of distraction and reappraisal. Journal of Cognitive Neuroscience. 1-15.

Meunier D, Lambiotte R, Fornito A, Ersche KD, Bullmore ET. 2009. Hierarchical modularity in human brain functional networks. Front Neuroinform. 3:37. 
Miranda-Dominguez O, Mills BD, Grayson D, Woodall A, Grant KA, Kroenke CD, Fair DA. 2014. Bridging the gap between the human and macaque connectome: a quantitative comparison of global interspecies structure-function relationships and network topology. J Neurosci. 34:5552-5563.

Mobbs D, Petrovic P, Marchant JL, Hassabis D, Weiskopf N, Seymour B, Dolan RJ, Frith CD. 2007. When fear is near: threat imminence elicits prefrontal-periaqueductal gray shifts in humans. Science. 317:1079-1083.

Modinos G, Ormel J, Aleman A. 2009. Activation of anterior insula during self-reflection. PLoS One 4: e4618.

Morecraft RJ, Geula C, Mesulam M-M. 1992. Cytoarchitecture and neural afferents of orbitofrontal cortex in the brain of the monkey. Journal of Comparative Neurology. 323:341-358.

Munkres J. 1957. Algorithms for the assignment and transportation problems. J Soc Ind Appl Math. 5:32-38.

Newman MEJ. 2006. Modularity and community structure in networks. Proc Natl Acad Sci USA. 103:8577-8582.

Nobre AC, Coull JT, Frith CD, Mesulam MM. 1999. Orbitofrontal cortex is activated during breaches of expectation in tasks of visual attention. Nature Neuroscience. 2(1):11-12.

Nomura EM, Reber PJ. 2008. A review of medial temporal lobe and caudate contributions to visual category learning. Neuroscience and Biobehavioral Reviews. 32: 279-291.

Ongür D, Price JL. 2000. The organization of networks within the orbital and medial prefrontal cortex of rats, monkeys and humans. Cereb Cortex. 10:206-219.

Pardo JV, Sheikh SA, Schwindt GC, Lee JT, Kuskowski MA, Surerus C, et al. 2008. Chronic vagus nerve stimulation for treatment-resistant depression decreases resting ventromedial prefrontal glucose metabolism. Neuroimage. 42: 879-89.

Parkes LM, Chan RCK, Kemp GJ, et.al. 2009. Depressive disorders: focally altered cerebral perfusion measured with arterial spin-labeling MR imaging. Radiology. 251(2):47684.

Paus, T., 2001. Primate anterior cingulate cortex: where motor control, drive and cognition interface. Nat. Rev. Neurosci. 2:417-424.

Pearson JM, Heilbronner SR, Barack DL, Hayden BY, Platt ML.2012. Posterior Cingulate Cortex: Adapting Behavior to a Changing Environment. Trends Cogn Sci. 15(4):143151.

Peng J, Liu J, Nie B, Li Y, Shan B, Wang G, Li K. 2011. Cerebral and cerebellar gray matter reduction in first-episode patients with major depressive disorder: a voxel-based morphometry study. Eur J Radiol. 80:395-9.

Pessiglione M, Seymour B, Flandin G, Dolan RJ, Frith CD. 2006. Dopamine-dependent prediction errors underpin reward-seeking behaviour in humans. Nature. 442:1042-5. 
Pezawas L, Meyer-Lindenberg A, Drabant EM, Verchinski B a, Munoz KE, Kolachana BS, et al. 2005. 5-HTTLPR polymorphism impacts human cingulate-amygdala interactions: a genetic susceptibility mechanism for depression. Nat Neurosci. 8:828-34.

Pizzagalli DA. 2010. Frontocingulate Dysfunction in Depression: Toward Biomarkers of Treatment Response. Neuropsychoph.1-24.

Ploner M, Lee MC, Wiech K, Bingel U, Tracey I. 2010. Prestimulus functional connectivity determines pain perception in humans. Proc Natl Acad Sci U S A. 107:355-360.

Porro CA, Cettolo V, Francescato MP, Baraldi P. 2003. Functional activity mapping of the mesial hemispheric wall during anticipation of pain. Neuroimage. 19:1738-1747.

Power JD, Barnes KA, Snyder AZ, Schlaggar BL, Petersen SE. 2012. Spurious but systematic correlations in functional connectivity MRI networks arise from subject motion. Neuroimage. 59:2142-2154.

Pourtois G, Vocat R, N'Diaye K, Spinelli L, Seeck M, Vuilleumier P. 2010. Errors recruit both cognitive and emotional monitoring systems: simultaneous intracranial recordings in the dorsal anterior cingulate gyrus and amygdala combined with fMRI. Neuropsychologia. 48:1144-1159.

Price JL, Drevets WC. 2010. Neurocircuitry of mood disorders. Neuropsychoph. 35:192-216.

Price JL, Drevets WC. 2012. Neural circuits underlying the pathophysiology of mood disorders. Trends Cogn Sci. 16:61-71.

Qiu L, Lui S, Kuang W, Huang X, Li J, Zhang J, et al. 2014. Regional increases of cortical thickness in untreated, first-episode major depressive disorder. Transl Psychiatry. 4:e378.

Raven J, Raven JC, Court JH. 2003. Manual for Raven's Progressive Matrices and Vocabulary Scales. Section 1: General Overview. San Antonio, TX: Harcourt Assessment.

Rey G, Desseilles M, Favre S, Dayer A, Piguet C, Aubry J-M, Vuilleumier P. 2014. Modulation of brain response to emotional conflict as a function of current mood in bipolar disorder: preliminary findings from a follow-up state-based fMRI study. Psychiatry Res. 223:8493.

Riva-Posse P, Holtzheimer PE, Garlow SJ, Mayberg HS. 2013. Practical considerations in the development and refinement of subcallosal cingulate white matter deep brain stimulation for treatment-resistant depression. World Neurosurg. 80:25-34.

Rolls ET. 2004. The functions of the orbitofrontal cortex. Brain Cogn. 55:11-29.

Rorden C, Brett M. 2000. Stereotaxic display of brain lesions. Behav Neurol. 12:191-200.

Roy M, Shohamy D, Wager TD. 2012. Ventromedial prefrontal-subcortical systems and the generation of affective meaning. Trends Cogn Sci. 16:147-56.

Rubinov M, Sporns O. 2010. Complex network measures of brain connectivity: uses and interpretations. Neuroimage. 52:1059-69. 
Rush AJ, Trivedi MH, Ibrahim HM, Carmody TJ, Arnow B, Klein DN, Markowitz JC, Ninan PT, Kornstein S, Manber R, Thase ME, Kocsis JH, Keller MB. 2003. The 16-item Quick Inventory of Depressive Symptomatology (QIDS) Clinician Rating (QIDS-C) and SelfReport (QIDS-SR): A psychometric evaluation in patients with chronic major depression. Biological Psychiatry. 54:573-583.

Salomons T V, Johnstone T, Backonja M-M, Davidson RJ. 2004. Perceived controllability modulates the neural response to pain. J Neurosci. 24:7199-203.

Samara Z, Evers EAT, Goulas A, Uylings HBM, Rajkowska G, Ramaekers JG, Stiers P. (submitted): Human orbitomedial prefrontal cortex: Intrinsic connectivity parcellation and functional organization.

Sambataro F, Wolf ND, Giusti P, Vasic N, Wolf RC. 2013. Default mode network in depression: A pathway to impaired affective cognition? Clin Neuropsychiatry. 10: 212216.

Seeley WW, Menon V, Schatzberg AF, Keller J, Glover GH, Kenna H, et al. 2007. Dissociable intrinsic connectivity networks for salience processing and executive control. J Neurosci. 27:2349-56.

Seminowicz DA, Mayberg H, Mclntosh a R, Goldapple K, Kennedy S, Segal Z, Rafi-Tari S. 2004. Limbic-frontal circuitry in major depression: a path modeling metanalysis. Neuroimage. 22: 409-18.

Sheline YI, Price JL, Yan Z, Mintun MA. 2010. Resting-state functional MRI in depression unmasks increased connectivity between networks via the dorsal nexus. Proc Natl Acad Sci. 107:11020-11025.

Singh-Curry V, Husain M. 2009. The functional role of the inferior parietal lobe in the dorsal and ventral stream dichotomy. Neuropsychologia. 47:1434-48.

Sohn MH, Albert M., Jung KJ, Carter CS, Anderson JR, 2007. Anticipation of conflict monitoring in the anterior cingulate cortex and the prefrontal cortex. Proc. Natl Acad. Sci. USA 104:10330-10334.

SPM 5: Welcome Trust Center for Neuroimaging, London, UK.

Stuhrmann A, Suslow T, Dannlowski U. 2011. Facial emotion processing in major depression: a systematic review of neuroimaging findings. Biol Mood Anxiety Disord. 1:10.

Sui R, Zhang L, Min L, Yuan J, Li X. 2009. Cerebellar dysfunction may play an important role in post-stroke depression. Med. Hypotheses. 72:643-646.

Sundermann B, Pfleiderer B. 2014. Meta-analysis of resting-state fMRI in depression Peer J PrePrints: $1-25$ doi.org/10.7287/peerj.preprints.412v1.

Takahashi T, Yücel M, Lorenzetti V, Tanino R, Whittle S, Suzuki M, et al. 2010. Volumetric MRI study of the insular cortex in individuals with current and past major depression. $J$ Affect Disord. 121:231-8.

Talairach T, Tournoux P. 1988. Co-planar stereotaxic atlas of the human brain. Thieme Medical Publishers Inc: New York. 
Tricomi E, Fiez JA. 2008. Feedback signals in the caudate reflect goal achievement on a declarative memory task. Neuroimage. 41:1154-1167.

Tzourio-Mazoyer N, Landeau B, Papathanassiou D, Crivello F, Etard O, Delcroix N, Mazoyer $B$, Joliot M. 2002. Automated anatomical labeling of activations in SPM using a macroscopic anatomical parcellation of the MNI MRI single-subject brain. Neuroimage. 15:273-289.

Upadhyay J, Maleki N, Potter J, Elman I, Rudrauf D, Knudsen J, et al. 2010. Alterations in brain structure and functional connectivity in prescription opioid-dependent patients. Brain. 133: 2098-114.

Ursu S, Clark KA, Aizenstein HJ, Stenger VA, Carter CS. 2009. Conflict-related activity in the caudal anterior cingulate cortex in the absence of awareness. Biol. Psychol. 80:279286.

Van Dijk KRA, Hedden T, Venkataraman A, Evans KC, Lazar SW, Buckner RL. 2010. Intrinsic functional connectivity as a tool for human connectomics: theory, properties, and optimization. J Neurophysiol. 103:297-321.

Van Dijk KRA, Sabuncu MR, Buckner RL. 2012. The influence of head motion on intrinsic functional connectivity MRI. Neuroimage. 59:431-438.

Van Tol M-J, Li M, Metzger CD, Hailla N, Horn DI, Li W, et al. 2013. Local cortical thinning links to resting-state disconnectivity in major depressive disorder. Psychol Med.1-13.

Vasic N, Walter H, Höse A, Wolf RC. 2008. Gray matter reduction associated with psychopathology and cognitive dysfunction in unipolar depression: a voxel-based morphometry study. J Affect Disord. 109:107-16.

Vincent JL, et al. 2006. Coherent spontaneous activity identifies a hippocampalparietal memory network. J Neurophysiol. 96:3517-3531.

Viviani R. 2013.Emotion regulation, attention to emotion, and the ventral attentional network. Front Hum Neurosci. 7: 746.

Wang L, LaBar KS, Smoski M, Rosenthal MZ, Dolcos F, Lynch TR, et al. 2008. Prefrontal mechanisms for executive control over emotional distraction are altered in major depression. Psychiatry Res.163:143-55.

Wang L, Hermens DF, Hickie IB, Lagopoulos J. 2012. A systematic review of resting-state functional-MRI studies in major depression. J Affect Disord. 142:6-12.

Yeo BT, Krienen FM, Sepulcre J, Sabuncu MR, Lashkari D, Hollinshead M, Roffman JL, Smoller JW, Zöllei L, Polimeni JR, Fischl B, Liu H, Buckner RL. 2011. The organization of the human cerebral cortex estimated by functional connectivity. J Neurophysiol. 106:1125-1165.

Yeterian EH, Pandya DN, Tomaiuolo F, Petrides M. 2012. The cortical connectivity of the prefrontal cortex in the monkey brain. Cortex. 48:58-81.

Zaki J, Ochsner KN, Hanelin J, Wager TD, Mackey SC. 2007. Different circuits for different pain: patterns of functional connectivity reveal distinct networks for processing pain in self and others. Social neuroscience. 2:276-291. 
Zeng L-L, Shen H, Liu L, Wang L, Li B, Fang P, et al. 2012. Identifying major depression using whole-brain functional connectivity: a multivariate pattern analysis. Brain. 135:1498507.

Zhang J, Wang J, Wu Q, Kuang W, Huang X, He Y, Gong Q. 2011. Disrupted brain connectivity networks in drug-naive, first-episode major depressive disorder. Biol Psychiatry. 70:334-42. 

Chapter 4

Altered face processing in Major Depression Vulnerability: Inhabiting different social worlds?

Zoe Samara, Elisabeth A.T. Evers, Johannes G. Ramaekers and Peter Stiers 


\section{Abstract}

Neuroimaging abnormalities during the processing of faces have been repeatedly reported in major depression (MD). However, it is not clear whether these abnormalities are mere correlates of the depressive episode or precede the onset of symptoms and possibly confer vulnerability to MD. We compared a group of participants with $M D(N=28)$, a group of individuals with familial MD vulnerability $(\mathrm{N}=30)$ and a group of matched controls $(\mathrm{N}=28)$. Participants were tested while passively viewing emotionally valenced (sad and happy) and neutral faces. We used functional magnetic resonance imaging (MRI) measures of blood oxygen level-dependent (BOLD) signal to quantify brain activity during face processing. By means of conjunction analyses we delineated the BOLD signal changes that were common to MD participants and participants with familial vulnerability compared to controls. These changes constitute vulnerability markers. BOLD changes associated with MD vulnerability were increases in the hippocampus and amygdala, lateral visual areas, the post and pre-central gyrus, the angular gyrus and the cerebellum. BOLD decreases were observed in frontal and cingulate areas, the thalamus and caudate nucleus, primary visual cortex, the angular gyrus and parts of the cerebellum. These changes were observed for all faces irrespective of affective valence. MD vulnerability is characterized by some of the most typical neurobiological abnormalities seen in the acute MD phase during face processing. Thus abnormalities in face processing precede the onset of the syndrome and might play a role in the development of MD. This study highlights the importance of human faces as stimuli to study affective processing in MD. 


\section{Introduction}

Human faces are highly significant stimuli of innate biological and social importance. The processing of emotional expressions on human faces is believed to be tightly linked to pathways that are also responsible for expressing affect facially (Adolphs, 2002; Anderson et al., 2011a) and for experiencing and regulating emotions (Bistricky et al., 2011). Given that, it is not surprising that behavioral and neurobiological abnormalities in affective face processing are found in many psychiatric syndromes, including major depression (MD). There is some behavioral evidence suggesting that abnormal processing of fearful (Bourke et al., 2010), sad (Bouhuys et al., 1999) and even neutral faces (Suslow et al., 2004; Leppanen et al., 2004) may be trait markers of MD (Kohler et al., 2011). Such abnormalities concern either impaired recognition of the depicted facial emotion (Gohier et al., 2014) or attentional biases towards and/or away from faces of particular valence (Gotlib et al., 2004; Joormann et al., 2007).

At the neurobiological level, studies employing $\mathrm{fMRI}$ during affective face processing implicate the amygdala, the insula, the parahippocampal and fusiform gyri, the putamen, the orbitofrontal cortex and parts of the cingulate gyrus in MD (reviewed in Stuhrmann et al., 2011). These studies have examined BOLD responses in acutely depressed patients. Thus, it is not clear whether these abnormalities are mere correlates of the depressive state or whether they precede the onset of episodes and possibly play a causal role in the development of MD. Studies that attempted to clarify this issue examined behavioral and neuroimaging abnormalities of affective face processing in remitted depressed and found evidence for the persistence of some of these abnormalities in the non-acute phase (Drevets et al., 1992; Joormann and Gotlib, 2007; Anderson et al., 2011b; Bhagwagar et al., 2004; Victor et al., 2011; Goulden et al., 2012) (for inconsistent findings see: Elliott et al., 2011; Leppanen, 2006; Thomas et al., 2011; Harmer et al., 2009; Fu et al., 2004; Sheline et al., 2001). While these studies dissociate abnormal face processing from the disease state, they cannot decisively answer whether changes in affective face processing preceded the onset of the symptoms since the possibility remains that the persisting abnormalities are disease "scars" or post-disease-onset adaptations.

To clarify this issue, efforts have been focused on whether affective face processing abnormalities are present in individuals susceptible to MD. Previous studies examined the effect of temperamental (neuroticism; Chan et al., 2009) or genetic predisposition (polymorphisms of the 5-HTTP serotonin 
transporter gene; Dannlowski et al., 2008; Del-Ben et al., 2008; other serotonergic genes; lidaka et al., 2005; Canli et al., 2008; catecholaminergic; Surguladze et al., 2012; Homan et al., 2014; noradrenergic; Neumeister et al., 2006) on face processing. Their results implicate several brain areas reported for the acute phase with the amygdala being the most consistently identified region. An alternative approach is to study samples of individuals who are at familial risk for MD (i.e. first-degree blood relatives of MD patients), without themselves having suffered an episode of depression. Thus far, evidence from at-familial-MD-risk individuals is limited. Monk et al. (2008) examined amygdala and nucleus accumbens responses to fear and happy faces in children and adolescent offspring of MD patients. In adults, van der Veen et al. (2007) found that tryptophan depletion led to stronger amygdala responses during face processing only in participants with familial history of MD. Recently, Miskowiak et al. (2015) found widespread changes in neural responses to fearful and happy faces in a small sample of monozygotic twins of depressed compared to twins of non-depressed siblings.

In the current study, we examine for the first time a large group of adult participants at familial risk for MD. Our aim was to examine BOLD signal changes during processing of sad, happy and neutral faces. More importantly, our approach is not limited to a comparison between MD vulnerable participants and healthy controls, but also includes a group of depressed participants. We specifically seek to identify face processing changes, relative to healthy controls, that are common to both patients and at-familiar-risk individuals, thus delineating putative MD endo-phenotypic markers.

\section{Methods}

\section{Design \& Participants}

Twenty-eight MD participants currently experiencing a major depressive episode (cutoff BDI-II > 20; mean age 39.2; SD 12.3; 68\% female), thirty unaffected first-degree MD relatives (mean age 33.2; SD 14.3; $73 \%$ female) and twenty-eight healthy control participants (mean age 35.5 ; SD $16.6 ; 71 \%$ female) took part in the study after providing informed consent. Patients were included if they met DSM-IV-TR criteria for major depressive disorder and excluded if they were diagnosed with Bipolar I or II, substance dependence or were taking benzodiazepines. Participants in the other two groups were excluded in the presence of any axis I diagnosis. Recruitment and screening procedures for all participants and diagnostic and medication 
histories of patients are reported in the Supplement. The study was approved by the Medical Ethical Committee of Maastricht University Medical Center.

During testing, participants completed the following self-report scales: Beck Depression Inventory-II (BDI-II), Brief Symptom Inventory (BSI), and the Quick Inventory of Depressive Symptomatology Self-Report (QIDS-SR) (Beck et al., 1996; Derogatis and Melisaratos, 1983; Rush et al., 2003). Participants were also assessed with a computerized version of Raven's Standard Progressive Matrices, a 60-item test of non-verbal IQ (Raven et al., 2003).

\section{Affective Faces Task}

Participants were instructed to categorize faces according to their gender (50\% female, 41 identities). Face stimuli were selected from the NimStim faces package based on the \% correct identification of the displayed emotion (sad, happy and neutral) (all faces $>80 \%$ ) by two independent samples (Tottenham et al., 2009; our own pilot). Affective faces were presented in blocks ( 32 secs) of either neutral and sad or neutral and happy faces ( 8 emotional and 4 neutral faces in each block). Face blocks were alternated with rest blocks ( 24 secs) during which participants were fixating on a cross in the middle of the screen (16 face and 16 rest blocks in total). Each face was presented in random order at the center of the computer screen against a gray background (E-prime) for 2 seconds. The interstimulus interval (cross fixation) varied randomly between $0,0.75,1.25$ or 2 seconds. Participants' reaction times and response accuracy were recorded.

\section{Image acquisition and preprocessing}

Scanning was conducted on a Siemens MAGNETOM Allegra 3T MRI head-only scanner. Head motion was constrained by the use of foam padding. For each subject, 680 T2*- weighted gradient echo planar images (EPI) with 33 slices were acquired. Imaging parameters for the EPI sequence were as follows: TR, $2000 \mathrm{~ms}$; TE, 25ms; flip angle, 90\%; matrix size, $128 \mathrm{X}$ 96; and FOV, 256mm; distance factor, $20 \%$; resulting in a voxel size of 2X2X3.24mm. A gradient echo image with the same grid and slice orientation as the functional images (TR, 704ms; TE, $5.11,7.57 \mathrm{~ms}$; flip angle, $60^{\circ}$ ) was used to generate a field map (offline correction of geometric distortions). In order to enable the localization of functional data, a high-resolution T1weighted image was acquired with the following parameters: TR, 2250ms; TE, 2.6ms; flip angle, 9'; FOV, $256 \mathrm{~mm}$; slice thickness, $1 \mathrm{~mm}$; matrix size, 
256X256; number of slices, 192; voxel size, 1X1X1mm. Echo time and voxel size of the EPI sequence were optimized for minimization of signal loss in orbital, medial frontal and temporal regions (susceptible to artefacts due to their proximity to air-tissue interfaces) that are of interest in face processing.

Preprocessing of fMRI data was performed using SPM 5 software (Welcome Trust Center for Neuroimaging, London, UK) and MATLAB (versions 7.9 and 8.2). The functional data were subjected to the following preprocessing: slice time correction, spatial correction using the field map, realignment, co-registration with the anatomical scan, normalization to the Montreal Neurological Institute (MNI) template, reslicing to $2 \mathrm{~mm}$ isotropic voxels and smoothing with a $6 \mathrm{~mm}$ full width half maximum (FWHM) Gaussian kernel. The T1-weighted images were segmented into grey matter, white matter and CSF tissue maps which were later used in the analyses.

\section{Statistical analyses}

Functional images were analyzed using the SPM 5 software (Welcome Trust Center for Neuroimaging, London, UK) and MATLAB. For each participant, general linear model (GLM) analysis was used to assess whole brain effects of task parameters on BOLD measures of activation. The first-level model included three experimental factors (sad, neutral and happy faces) with time and dispersion derivatives. As effects of no interest we included six realignment parameters, the averaged signal from white matter and CSF and trials in which the frame-wise displacement exceeded a set threshold (see Supplement for details). Low frequency signal drift was eliminated using a high-pass filter (cutoff 128-secs) and voxels' autocorrelations were corrected by an autoregressive model. During the first level analysis \% BOLD signal change maps for each face valence against baseline were created for every participant.

To compare brain activation during overall face processing between the three groups the \% BOLD signal change maps were entered in a secondlevel random-effects analysis, using a 1-way factorial design with one factor (MD, family MD history and healthy controls) and three covariates (age, gender and Raven IQ score). MD vulnerability at the brain level was defined as voxels, whose response did not differ between MD patients and familial MD participants, while it was significantly different in the comparison of both experimental groups with the healthy control participants. These MD vulnerability marking voxels were identified in two steps. We first created a conjunction map of two F contrasts: 1) the contrast map between healthy 
controls and the MD patients and 2) between the healthy controls and the familial MD participants. This conjunction map was tested for significance with Monte Carlo simulations (10.000 iterations; voxel-level $p=.01$, clusterlevel $p=.005)$. The second step was implemented to retain as vulnerability markers only voxels that did not differ significantly between MD patients and familial MD participants. Consequently, we removed from the conjunction map all voxels that were significant at a lenient threshold (.001 uncorrected) in the F contrast between MD patients and familial MD participants. We'll refer to this map as the corrected conjunction map for MD vulnerability.

Since ANOVA is a general test for differences between groups, we determined the direction of the BOLD differences in MD vulnerability with a post-hoc group based ROI analysis (IBM SPSS 21) of the voxel clusters retained in the above conjunction map. Finally, behavioral data were analyzed to test whether groups differed in accuracy and reaction time of gender categorization of the facial stimuli. For parameters and details of all analyses see Supplement. 


\section{Results}

\section{Demographic and Clinical Characteristics}

The familial MD group did not differ significantly in scores of depressive and general psychiatric symptomatology from the healthy controls (see Table 1 for this section). MD patients at the time of the scan had on average BDI-II and QIDS-SR scores of 32.32 and 16.14 respectively. Groups were matched in terms of age, gender or IQ (all p's >.2).

Table 1. Sample characteristics and clinical measures.

\begin{tabular}{|c|c|c|c|c|c|c|c|c|}
\hline & & & & & & & & \\
\hline & Mean & SD & Mean & SD & Mean & SD & $F(2,83)$ & $\begin{array}{c}\mathbf{p} \\
\text { Values }\end{array}$ \\
\hline BDI-II & 32.32 & 7.26 & 4.53 & 3.88 & 2.68 & 3.30 & 300.34 & $.000^{a}$ \\
\hline $\begin{array}{l}\text { BSI } \\
\text { GSI }\end{array}$ & 1.63 & 0.53 & 0.23 & 0.22 & 0.15 & 0.18 & 164.66 & $.000^{\mathrm{a}}$ \\
\hline $\begin{array}{l}\text { BSI } \\
\text { PST }\end{array}$ & 38.29 & 8.11 & 10.03 & 8.29 & 6.79 & 7.27 & 135.30 & $.000^{\mathrm{a}}$ \\
\hline $\begin{array}{l}\text { BSI } \\
\text { PSDI }\end{array}$ & 2.22 & 0.43 & 1.09 & 0.27 & 0.88 & 0.44 & 99.52 & $.000^{\mathrm{a}}$ \\
\hline QIDS & 16.14 & 3.16 & 3.03 & 2.22 & 2.25 & 2.01 & 274.00 & $.000^{\mathrm{a}}$ \\
\hline Age & 39.21 & 12.29 & 33.23 & 14.28 & 35.54 & 16.63 & 1.25 & .293 \\
\hline $\begin{array}{l}\% \\
\text { Female }\end{array}$ & $68 \%$ & --- & $73 \%$ & --- & $71 \%$ & --- & $X^{2}(2)=.22$ & .898 \\
\hline $\begin{array}{l}\text { Raven } \\
\text { IQ }\end{array}$ & 45.93 & 7.13 & 48.63 & 6.16 & 45.68 & 7.15 & 1.69 & .190 \\
\hline
\end{tabular}

$M D$, patient group; $F H$, at-familial risk group; $H C$, healthy controls; GSI, global severity index; PST, positive symptom total; PSDI, positive symptom distress index. ${ }^{a}$ The MD group differs significantly from relatives and controls.

Accuracy of gender categorization of the presented facial stimuli did not differ significantly among any of the groups $(p>.4)$. With regard to reaction time, MD participants were significantly slower $(F(2,83)=12.27, p=.000)$ from both the familial MD ( $p=.008$; post-hoc Bonferroni corrected) and the healthy 
control group ( $p=.000$; post-hoc Bonferroni corrected). The last two groups did not differ significantly from one another in reaction times $(p>2)$.

\section{BOLD markers of MD Vulnerability during face processing}

The corrected conjunction map revealed 27 independent clusters in which BOLD signal differed significantly voxel-wise between both the MD patient and familial MD groups compared to healthy controls during face processing. ROI-based post-hoc tests of these clusters determined the direction of BOLD signal changes. Table 2 reports the anatomical location, local maxima, coordinates, $p$ values and size in voxels for all clusters with differential activation in MD vulnerability. 
Table 2. Summary table of MRI results

\section{Anatomy}

Cerebellum $\mathrm{R}$

Fusiform L

Hippocampus/Amygdala R

Temporal Inferior L

Temporal Mid R

Temporal Inferior R

Angular $\mathrm{R}$

Post-central L

Pre/Post-central R

Post-central L

Post-central R

Cerebellum L (Crus I)

Cerebellum R (6)

Cerebellum L (Crus I)

Calcarine L

Calcarine $\mathrm{L}$

Calcarine $\mathrm{R}$

Lingual/Vermis L

Thalamus L

Frontal (S/M/O) R

Caudate $\mathrm{R}$

Lingual $\mathrm{R}$

Lingual L

Cuneus L

Angular L

Cingulate Mid L

Frontal Mid L
ROI $F$ value (local maximum)

13.00

17.71

17.54

12.51

27.22

15.29

14.36

14.32

15.19

21.28

27.64

21.15

11.98

22.55

13.23

12.92

39.04

11.73

17.27

14.07

14.63

13.91

13.25

19.58

10.97

12.70

13.86
MNI

(loc.max)

14, $-36,-20$

$-40,-76,-16$

$18,-4,-16$

$-58,-36,-16$

$54,-74,-6$

$56,-50,-6$

$32,-62,32$

$-36,-44,36$

$46,-28,42$

$-50,-18,42$

$56,-20,44$

$-44,-50,-36$

$22,-74,-24$

$-6,-82,-16$

$6,-92,-6$

$-10,-90,-2$

$18,-74,-2$

$-2,-68,-2$

$-14,-12,2$

$26,68,2$

$14,18,0$

4, -66, 2

$-30,-64,4$

$-10,-92,38$

$-40,-64,36$

$-10,8,38$

$-36,32,40$ p Value

.0006

.0041

.0013

.0015

.0012

.0096

.0025

.0005

.0003

.0005

.0003

.0083

.0145

.0099

.0062

.0537

.0003

.0143

.0000

.0013

.0002

.0235

.0058

.0019

.0153

.0079

.0021
Size in

voxels

34

64

46

74

137

39

44

65

126

154

156

33

34

44

50

27

425

27

57

54

36

35

32

24

39

31

88 
Anatomy labels correspond to AAL (Tzourio-Mazoyer et al., 2002); red corresponds to areas with increased $B O L D$ signal in $M D$ vulnerability while green corresponds to areas with decreased signal (see also Figure 1). $P$ values correspond to overall $F$ test (see Supplementary table 2 for $p$ values of post-hoc comparisons).

MD vulnerability markers (Figure 1 ) were exaggerated reactions of the right hippocampus and amygdala, enhanced responses of lateral visual areas (left fusiform gyrus and right inferior and mid temporal cortex) and increased responses in somatosensory and motor regions of the pre and post-central gyrus bilaterally. MD vulnerability was also characterized by weakened responses in the left mid-cingulate and dorsolateral prefrontal cortex in both hemispheres, and primary visual areas (left and right calcarine and lingual and left cuneus). In addition, weakened reactions were observed in the left thalamus and the head of the right caudate nucleus. MD vulnerability was also associated with BOLD signal changes (both increases and decreases compared to controls) in regions of the cerebellum. Finally we observed a laterality effect in the inferior parietal cortex; in MD vulnerability the right angular gyrus showed increased BOLD responses during face processing while the left angular showed the opposite pattern.
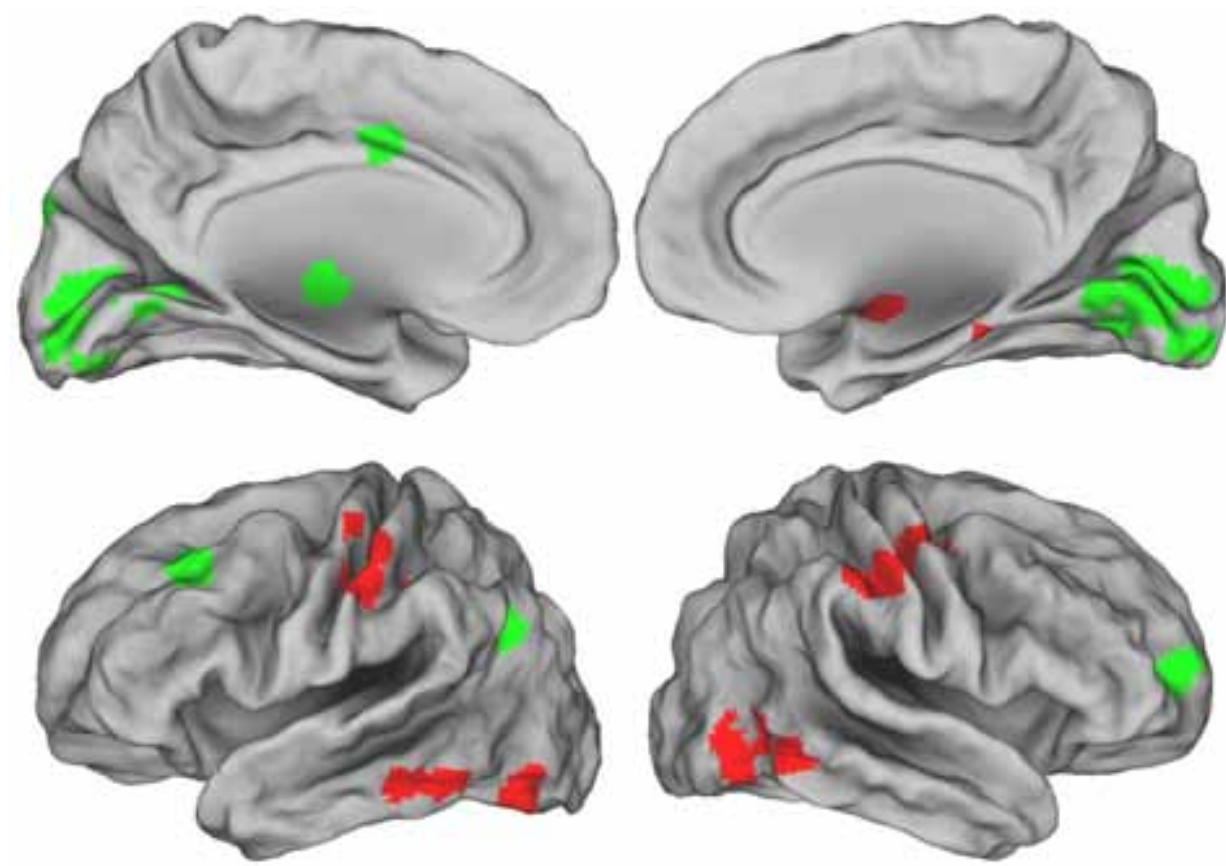

Figure 1. Surface representation (CARET; Pals B12 Fiducial) of main results. Red plots regions with increased \% BOLD signal in MD vulnerability (MD patients and at- 
familial-risk participants) compared to controls. Green plots decreases of signal in the same comparison. Not all clusters are shown on the surface, for MNI coordinates see Table 2.

For all regions reported here showing altered responses in $M D$ vulnerability to faces, the direction of the change in BOLD signal was uniform across the face valence categories (i.e. no area shows a different pattern for sad, neutral and happy faces). For illustration purposes, Figure 2 plots the mean \% BOLD signal change of the amygdala and the angular gyrus bilaterally across all faces and for each valence separately (see Supplement for complete data).

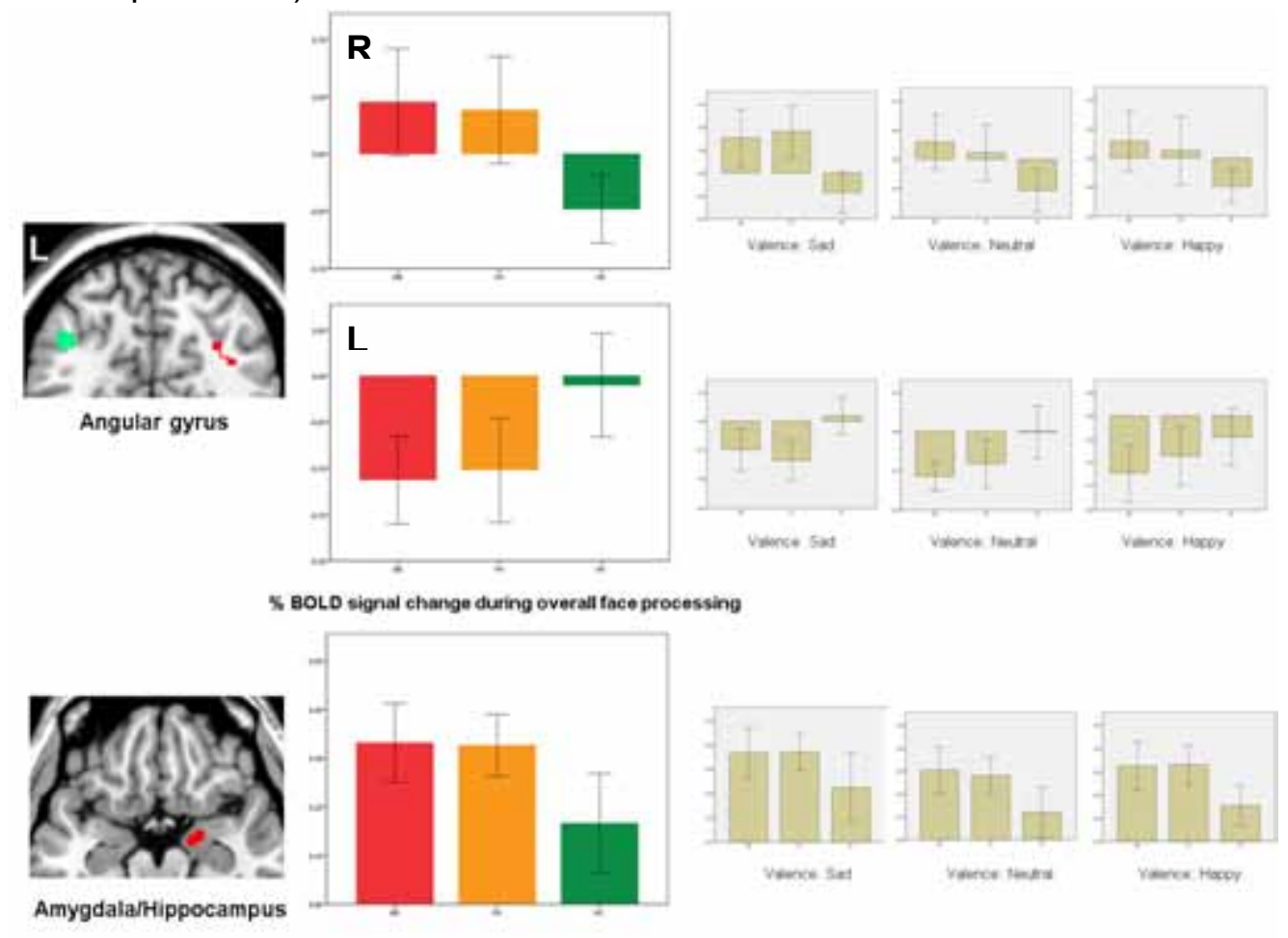

Figure 2. Bar plots (error bars signify $95 \%$ confidence intervals) of the effects in the angular gyrus bilaterally and the right amygdala and hippocampus. Red color bars correspond to the MD patient group, orange to the familial MD group and green to healthy controls. Main graph y axes range from top to bottom: -0.1 to $.01 ;-0.2$ to .05 ; 0.0 to 0.5 . Right most panels plot the $\%$ BOLD signal change for each face valence separately to demonstrate the uniformity of effects (for all data see Supplement). 


\section{Discussion}

This study delineated differences during face processing in MD vulnerability contrasting a group of familial MD participants and MD patients with healthy controls. A conjunction analysis revealed BOLD response markers of MD vulnerability reflecting three main patterns. First, enhanced BOLD reactions were observed for MD vulnerability in the hippocampus and amygdala, lateral visual areas and somatosensory and motor regions. Second, decreased BOLD responses were found in frontal and primary visual areas, the caudate and the thalamus. Finally, the angular gyrus exhibited a lateralized effect of decreased left and increased right BOLD response during face processing in MD vulnerability. These data are the first to demonstrate face processing abnormalities in at familial risk adults similar to those reported in the acute and remitted MD phase. These changes were found for all faces (sad, neutral and happy), thus irrespective of their affective valence. Behaviorally, attention and cognitive performance of the MD patient and MD familial vulnerability groups was comparable to healthy controls (accuracy of gender categorization did not differ significantly among any of the groups) while MD patients were slower to respond compared to the other two groups, in line with studies finding a general tardiness in patients' responses (Buyukdura et al. 2010). We found that MD vulnerability is characterized by exaggerated BOLD responses in the hippocampus and the amygdala during face processing. Our finding is in agreement with previous studies showing that MD adult patients have increased hippocampal responses to masked sad facial stimuli (Victor et al. 2010, 2012) and that increased hippocampal response to sad faces is associated with depression severity in pre-school onset MD (Barch et al. 2012). On the other hand, contrary to our finding, Lee et al. (2009) have reported decreased activity in the hippocampus for sad faces vs baseline. During facial processing the hippocampus matches perceptual characteristics to memory representations and activates the associations triggered by the incoming stimuli, thus providing input to the amygdala about the environmental context (Victor et al., 2010).

Abnormalities in amygdala's response to faces is suggested to be a trait MD marker (Victor et al., 2010; Arnone et al. 2012), mediated by serotonergic (Anderson et al. 2007, 2011; Dannlowski et al. 2008; Lau et al. 2009; Lee et al. 2009; Hornboll et al. 2013; Ruhé et al. 2014) and noradrenergic (Neumeister et al. 2006; Henderson et al. 2014) interactions. Amygdala's role in face processing is believed to be the assessment of the stimuli's biological relevance and the evaluation of the stimuli-emotional 
significance associations (Leppänen and Nelson 2009). However, it is still under debate whether amygdala responds predominantly to negative and in particular threatening stimuli such as fear and anger in MD, as well as whether BOLD signal abnormalities in the amygdala are of opposite direction in response to negative versus positive stimuli. Here we report differences in the right amygdala between MD vulnerability (patients and at risk individuals) and the healthy control group for sad, neutral and happy faces. Thus our results suggest that amygdala in MD vulnerability is hyper-activated in response to all presented stimuli, irrespective of their affective valence, signifying, thus, an overall emotional or alert reaction. In line with this, in a sample of adolescents with depressive and/or anxiety disorder van den Bulk et al. (2014), reported a positive correlation between anxiety symptoms and $\%$ BOLD signal change in right amygdala for fearful, neutral and happy faces compared to fixation. Gaffrey et al. (2011) found evidence for a stronger activation of the right amygdala in depressed preschoolers in response to happy faces. Interestingly, he also found evidence for the same effect in neutral faces when analysis was restricted to a smaller group of the sample with less movement. In addition to that, we report differences in the right amygdala in the same direction for sad and happy faces. This finding is at odds with studies reporting BOLD increases for sad faces vs baseline and BOLD decreases for happy faces vs baseline in the amygdala (Suslow et al. 2010; Victor et al. 2010; Stuhrmann et al. 2013) but agrees with studies reporting uniform effects in the MD acute phase (Sheline et al. 2001; Dannlowski et al. 2009; Lau et al. 2009). Similarly for MD vulnerability, Dannlowski et al. (2008) found that MD patients with the s allele of the 5HTTLPR gene had stronger amygdala reactions not only to negative (sad and angry) but also to happy faces. Similar effects have been reported for $s$ allele carriers with panic disorder (Domschke et al., 2006).

The amygdala is reciprocally connected to higher-tier visual areas (Zhang et al. 2015). According to Leppänen and Nelson (2009), evidence from the processing of emotional stimuli supports a model in which the amygdala responds to coarse information about facial expressions at the early stages of processing and via back-projections enhances subsequent detailed perceptual processing in the fusiform and temporal cortex. In agreement with this proposition, we also observed increased BOLD responses in the fusiform gyrus and inferior temporal cortex in MD vulnerability. Our finding of increased BOLD activation in the fusiform is in agreement with previous studies reporting increased activation to sad faces 
(Keedwell et al. 2005; Surguladze et al. 2005; Suslow et al. 2010) in MD patients although decreases in activity (Fu et al. 2008) and differential response based on affective valence (Surguladze et al., 2005; Suslow et al., 2010) have also been reported. With regard to the inferior temporal cortex in MD, Canli et al. (2008) found that activation of the inferior temporal cortex in the fear versus baseline contrast of faces was positively correlated with having the combined 5-HTTLRP and TPH-2 vulnerability genotypes.

According to neurobiological models of emotional face processing, the somatosensory cortex is part of an extended system of brain areas involved in processing the meaning and significance of facial information (Stuhrmann et al., 2011). It is speculated that processing of faces with intense emotional expressions activates representations of the perceived emotion in the individual (Adolphs, 1999; Haxby et al., 2000) and the somatosensory cortex codes the physical aspect of the emotional state or feeling. Many studies report aberrant activations and connectivity of the somatosensory cortex in MD, likely related to altered emotional reactivity or experience at its most basic level. Victor et al. (2012) reported increased BOLD responses in the post-central gyrus of MD patients for masked-happy vs masked-neutral faces. Here we report increased activation in the pre and postcentral gyrus in MD vulnerability, consistent with previous studies in MD patients (Fu et al., 2004; Keedwell et al. 2005; Frodl et al. 2008; Fu et al. 2008; Scheuerecker et al. 2010).

Parallel to increased BOLD signal changes in the aforementioned regions, we observed diminished responses in a number of areas in MD vulnerability, namely in midline visual areas, the dorsolateral frontal and cingulate cortex as well as the thalamus and caudate nucleus. Low-tier visual regions in the lingual gyrus, calcarine fissure and the cuneus have been repeatedly implicated in the MD pathology (Zeng et al., 2012), although the exact role of these abnormalities remains unclear. A large meta-analysis of 105 imaging studies (Fusar-Poli et al. 2009) reported that the visual cortex is activated during face processing in healthy controls across all emotional conditions. It is plausible that MD vulnerability impacts early perceptual processing of facial stimuli (Fusar-Poli et al., 2009). Alternatively, differential BOLD signal changes in these areas might underlie differences in attention or the reward value of the incoming stimuli (Stănişor et al. 2013) between groups. The midcingulate is involved in signaling predictions and error monitoring also in social interactions (Apps et al. 2013).Here we report decreased activation in MD vulnerability which might contribute to altered 
perception or representation of social interactions and the associated expectations and thus social decision making. Previous studies has reported decreased mid-cingulate activation in MD for sad faces vs baseline (Fu et al. 2008; Pan et al. 2013), while increases have also been reported (Fu et al., 2004; Frodl et al., 2009; Keedwell et al., 2005). Although the resolution at $3 T$ does not enable precise localization of thalamic nuclei, the cluster reported here appears to fall within the ventrolateral region of the thalamus, a region involved in motor functions (Anderson et al. 2006) and sensory processing (Ro et al., 2007;Ro 2010). According to Victor et al. (2012), the sensory part of the thalamus together with the amygdala, function to modulate neural and behavioral responses to emotional stimuli on the basis of learning, context and changing reinforcement contingencies. The thalamus has been found to show both less (Lawrence et al. 2004) and more (Fu et al., 2004; Mingtian et al. 2012; Victor et al. 2012) activation in MD patients during face processing. The caudate is considered part of the extended network associated with emotional aspects of faces (Arsalidou et al. 2010). The caudate is known to play a role in motor control (Menon, Glover, \& Pfefferbaum, 1998), learning visual categories (Nomura \& Reber, 2008) and processing reward-related information (Tricomi and Fiez 2008). Findings in MD patients during facial processing in the caudate are mixed, with studies reporting both increased (Fu et al., 2004; Scheuerecker et al., 2010) and decreased (Lawrence et al., 2004; Lee et al., 2008;) responses. Murrough et al. (2015) found reduced activation of the right caudate in treatment resistant patients to happy faces, which normalized after a single dose of ketamine. In healthy groups, mirtazapine (acting on serotonine and noradrenergic receptors) also modulated activation of the caudate in response to facial expressions (Rawlings et al. 2010). Finally, we report here BOLD increases and decreases of various cerebellar regions and the angular gyrus in MD vulnerability. The cerebellum remains a largely unexplored cortical area, mostly known for its involvement in motor function. However, there is evidence implicating the cerebellum both in MD and in emotional face processing (Chan et al. 2009). Recently, Ferrucci et al. (2012) demonstrated that transcranial direct current stimulation of the cerebellum enhanced sensory processing of facial expressions. The angular gyrus and particularly the left, appears to participate in the processing of facial characteristics (Kesler-West et al. 2011) such as the ones which decode age on human faces (Homola et al. 2012) and has been previously implicated in MD (Gaffrey et al., 2013; Zeng et al. 2012). The angular gyrus is also part of the 
default mode network and the cortical-limbic circuits and is thought to play a role in emotional modulation (Zeng et al., 2012). Here we report differential responses to faces in the left and right angular gyrus, with left angular showing decreased and right angular gyrus showing increased BOLD response during face processing.

Limitations of our study might be lack of power to detect effects for each affective valence separately and examination of BOLD responses only for sad and happy faces (it remains open whether the effects generalize to other types of negative and positive emotions). In addition, our neutral faces were presented in blocks of emotional faces (either sad or happy); given that, it is possible that some of the effects we report here for the neutral faces are confounded by the fact our neutral stimuli were embedded in either negative or positive context. Finally, our study cannot answer the question whether abnormalities at the neurobiological level translate to behavioral effects in MD vulnerability since the task participants were asked to perform was simple gender categorization.

In conclusion, we observed a pattern of BOLD signal increases in limbic and lateral visual areas and decreases in thalamic, striatal, cingulate, and primary occipital regions during face processing. These areas have been consistently identified before as being relevant to MD. We demonstrate for the first time in this report that these abnormalities are found not only in MD patients but are also shared by adults at familial risk for depression. 


\section{Supplement}

\section{Design \& Participants}

Twenty-eight MD participants currently experiencing a major depressive episode (cutoff BDI-II > 20; mean age 39.2; SD 12.3; 68\% female), thirty unaffected first-degree MD relatives (mean age 33.2; SD 14.3; $73 \%$ female) and twenty-eight healthy controls (mean age 35.5; SD 16.6; $71 \%$ female) participated in the study after providing informed consent. The three groups did not differ significantly in terms of age $(F(2,83)=1.25$; $p=.29)$, gender $(X 2(2)=0.22 ; p=.90)$ or IQ $(F(2,83)=1.69 ; p=.19)$. Nonetheless, to further remove variability associated with these parameters, we included them as covariates in our fMRI analyses.

Participants in all three groups were recruited via advertisements in the local press; MD patients were also recruited from the regional institute for outpatient mental healthcare (RIAGG, Maastricht NL). MD patients recruited from the community were screened for axis I disorders with SCID-I (DSM-IV structured clinical interview) (1) and BDI-II by the experimenter (Clinical Psychologist, MSc.) and trained research assistants. Outpatients of the regional institute for mental healthcare were interviewed for axis I disorders with SCID-I at their intake at the center. All aspects of our screening and experimental procedure were approved by the Medical Ethical Committee of the Academic hospital of Maastricht University and conducted in accordance with the University's and Committee's guidelines.

MD patients were included if they met DSM-IV-Text Revision criteria for major depressive disorder as the primary diagnosis and had a BDI-II score of $>20$ (moderate to severe depression) and excluded if they met criteria for Bipolar I or II, substance dependence or were taking benzodiazepines. MD patients with additional axis I diagnoses (except substance dependence and psychotic disorders) or taking other classes of antidepressants were allowed to participate. Table 1 details the diagnostic and medication histories of MD participants. To exclude the possibility that our main results were driven by comorbidity and medication use in part of our MD sample we filtered out from our analyses voxels significant in associated comparisons (for details see section on MRI analyses).

Participants with familial MD were included if they had a first-degree relative with $M D$, as detailed in a screening questionnaire administered to them. The questionnaire included DSM-IV items for the diagnosis of MD and the differential diagnosis of Bipolar I, II and psychotic features. Participants in 
this group were excluded if they had been personally diagnosed with any axis I disorder and were assessed with the Symptoms-Checklist 90 (SCL-90) (2) for current psychiatric symptomatology (exclusion cutoff for males SCL-90 > 116, females SCL-90 > 130; above average population norms).

Healthy controls were excluded if they had ever been diagnosed with any axis-I disorder and if they had a first or second degree relative with psychiatric history. They were assessed with the SCL-90 for current psychiatric symptomatology and were excluded based on the same cutoffs as the familial MD group. All participants were screened for the following somatic conditions and MRI contra-indications: neurological disorders, epilepsy, severe head injury, claustrophobia, pregnancy or lactation, metal implants, pacemakers and intrauterine contraceptive devices.

\section{Magnetic Resonance Imaging Acquisition \& Preprocessing}

Preprocessing of fMRI data was performed using SPM 5 software (3) and MATLAB scripts (Mathworks, Natick, MA, USA). The functional data were subjected to the following preprocessing: slice time correction, spatial correction using the field map, realignment, co-registration with the anatomical scan, normalization to the Montreal Neurological Institute (MNI) template, reslicing to $2 \mathrm{~mm}$ isotropic voxels and smoothing with a $6 \mathrm{~mm}$ full width half maximum (FWHM) Gaussian kernel. The T1-weighted images were segmented into grey matter, white matter and CSF tissue maps which were later used in the analyses.

During the preprocessing step we calculated various metrics of framewise displacement and excluded from further analysis all subjects with translation distance $>3 \mathrm{mms}$. In order to reduce the bias of head motion in our data we took the following approach: 1) we estimated the scan-to-scan head motion and identified scans during which the frame-wise displacement exceeded a particular threshold (absolute motion difference in the $z$ direction $>0.4 \mathrm{~mm}\left(1 / 10\right.$ of voxel size); rotation in the $x$ direction $>0.26^{\circ}$ (angle corresponding to $0.4 \mathrm{~mm} z$-displacement of frontopolar voxels, assuming the rotation point in the middle of the brain is $88 \mathrm{~mm}$ from the anterior end of the brain's frontal pole; Talairach and Tournoux, 1988)), 2) we marked and excluded the identified volumes together with the 1-back and the 2-forward frames (to avoid spin history assumptions' violations caused by movement; Power et al., 2012), and 3) we included in the analyses only participants for whom more than $25 \%$ (corresponding to 48 volumes) of the event trials were removed. Finally, to ensure that group BOLD effects would not be attributable 
to movement, we compared and equated the three groups on the total number of removed trials and the mean relative translation distance after cleaning (resulted in exclusion of one participant of the MD group with the maximum mean relative translation distance). 


\section{Supplementary Figures \& Tables}

Supplementary Table 1. Demographic and clinical data of the MD patient group.

\begin{tabular}{|c|c|c|c|c|}
\hline Age & Gender & Axis I Comorbidity & $\begin{array}{l}\text { Current } \\
\text { Medications }\end{array}$ & Med Type \\
\hline 22 & $\mathrm{~F}$ & & & \\
\hline 31 & $\mathrm{~F}$ & PTSD & Venlafaxine & SNRI \\
\hline 59 & $\mathrm{~F}$ & & & \\
\hline 49 & M & Social Phobia & & \\
\hline 34 & $\mathrm{~F}$ & & & \\
\hline 18 & $\mathrm{~F}$ & & Citalopram & SSRI \\
\hline 25 & $\mathrm{~F}$ & Social Phobia & Sertraline & SSRI \\
\hline 49 & $\mathrm{~F}$ & & Paroxetine & SSRI \\
\hline 20 & $M$ & PTSD & & \\
\hline 52 & M & & & \\
\hline 31 & $\mathrm{~F}$ & & Nortriptyline & TCA \\
\hline 23 & $\mathrm{~F}$ & Specific Phobia & Bupropion & NDRI \\
\hline 52 & $\mathrm{~F}$ & Specific Phobia & Citalopram & SSRI \\
\hline 47 & M & & $\begin{array}{l}\text { Venlafaxine, } \\
\text { Seroquel }\end{array}$ & $\begin{array}{l}\text { SNRI, } \\
\text { Antipsychotic }\end{array}$ \\
\hline 44 & $\mathrm{M}$ & & $\begin{array}{l}\text { Citalopram, } \\
\text { Mirtazapine }\end{array}$ & SSRI, TetraCA \\
\hline 25 & $\mathrm{~F}$ & & Agomelatine & Melatonergic \\
\hline 36 & $\mathrm{~F}$ & & & \\
\hline 39 & $\mathrm{~F}$ & Social Phobia, GAD & & \\
\hline 52 & M & & & \\
\hline 44 & M & GAD & Paroxetine & SSRI \\
\hline 42 & $\mathrm{~F}$ & Panic Disorder with agoraphobia & Citalopram & SSRI \\
\hline 54 & $\mathrm{~F}$ & & & \\
\hline 55 & $\mathrm{~F}$ & PTSD & Citalopram & SSRI \\
\hline 49 & $\mathrm{~F}$ & & & \\
\hline 37 & M & Specific Phobia, Social Phobia & & \\
\hline 38 & $\mathrm{~F}$ & Social Phobia & & \\
\hline 44 & $\mathrm{~F}$ & & & \\
\hline 36 & $\mathrm{~F}$ & Social Phobia & & \\
\hline 38 & $\mathrm{~F}$ & & Escitalopram & SSRI \\
\hline 22 & $\mathrm{~F}$ & & & \\
\hline 19 & $\mathrm{~F}$ & $\begin{array}{l}\text { GAD, Panic with agoraphobia, } \\
\text { ADHD/ADD }\end{array}$ & Citalopram & SSRI \\
\hline 34 & $\mathrm{M}$ & $\begin{array}{l}\text { Panic Disorder with agoraphobia, Social } \\
\text { Phobia }\end{array}$ & Escitalopram & SSRI \\
\hline 43 & M & Social Phobia & & \\
\hline 54 & $\mathrm{~F}$ & Agoraphobia & & \\
\hline 44 & $\mathrm{~F}$ & Panic disorder, OCD & Fluvoxamine & SSRI \\
\hline
\end{tabular}


Abbreviations: PTSD (Post Traumatic Stress Disorder); GAD (Generalized Anxiety Disorder); ADHD/ADD (Attention Deficit Hyperactivity Disorder); OCD (Obsessive Compulsive Disorder); SNRI (Serotonin Norepinephrine Reuptake Inhibitor); SSRI (Selective Serotonin Reuptake Inhibitor); TCA (Tricyclic Antidepressant); NDRI (Norepinephrine Dopamine Reuptake Inhibitor); TetraCA (Tetracyclic Antidepressant).
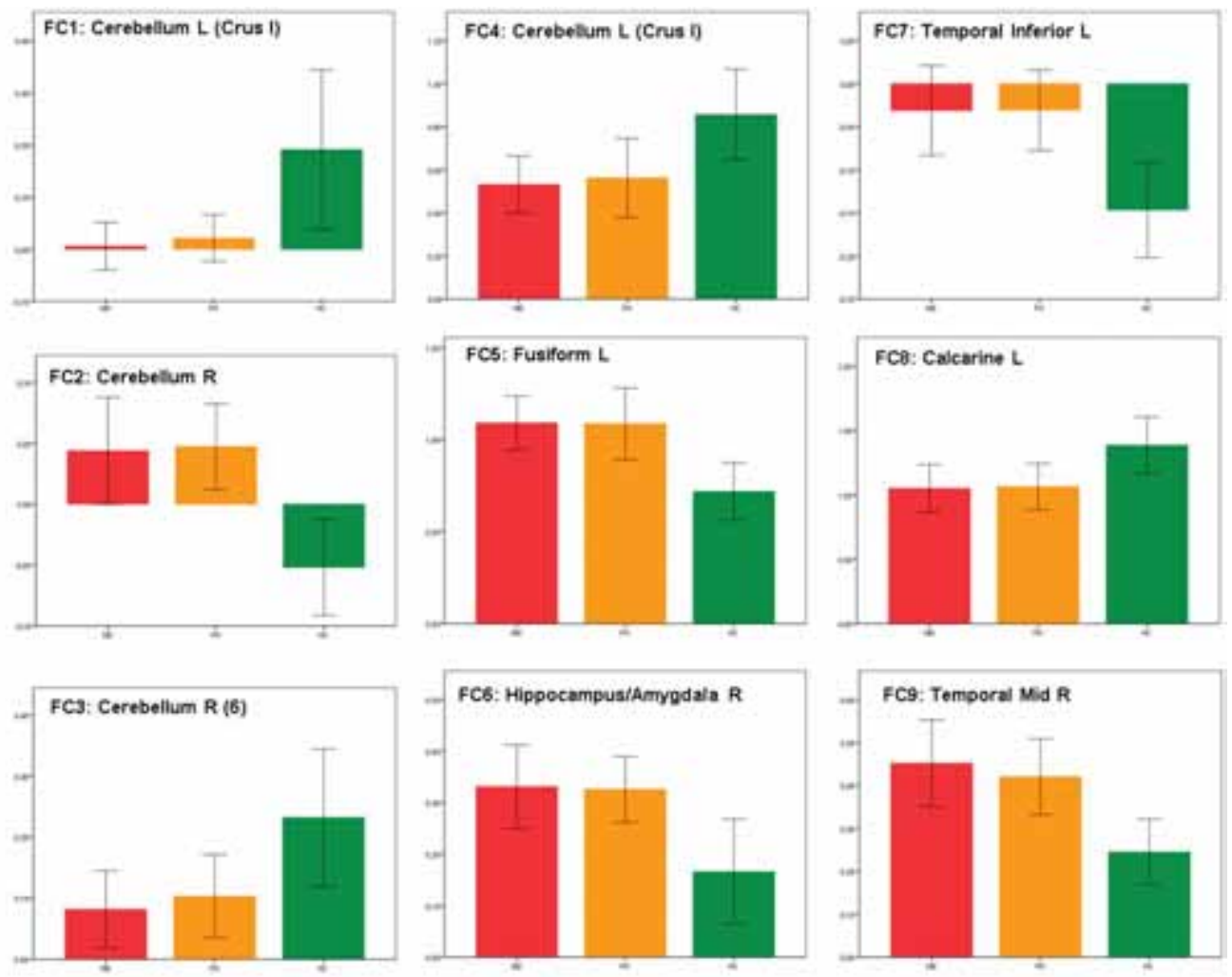

Supplementary Figure 1. Bar plots (error bars signify 95\% confidence intervals) for all effects. Red color bars correspond to the MD patient group, orange to the familial $M D$ vulnerability group and green to healthy controls. 

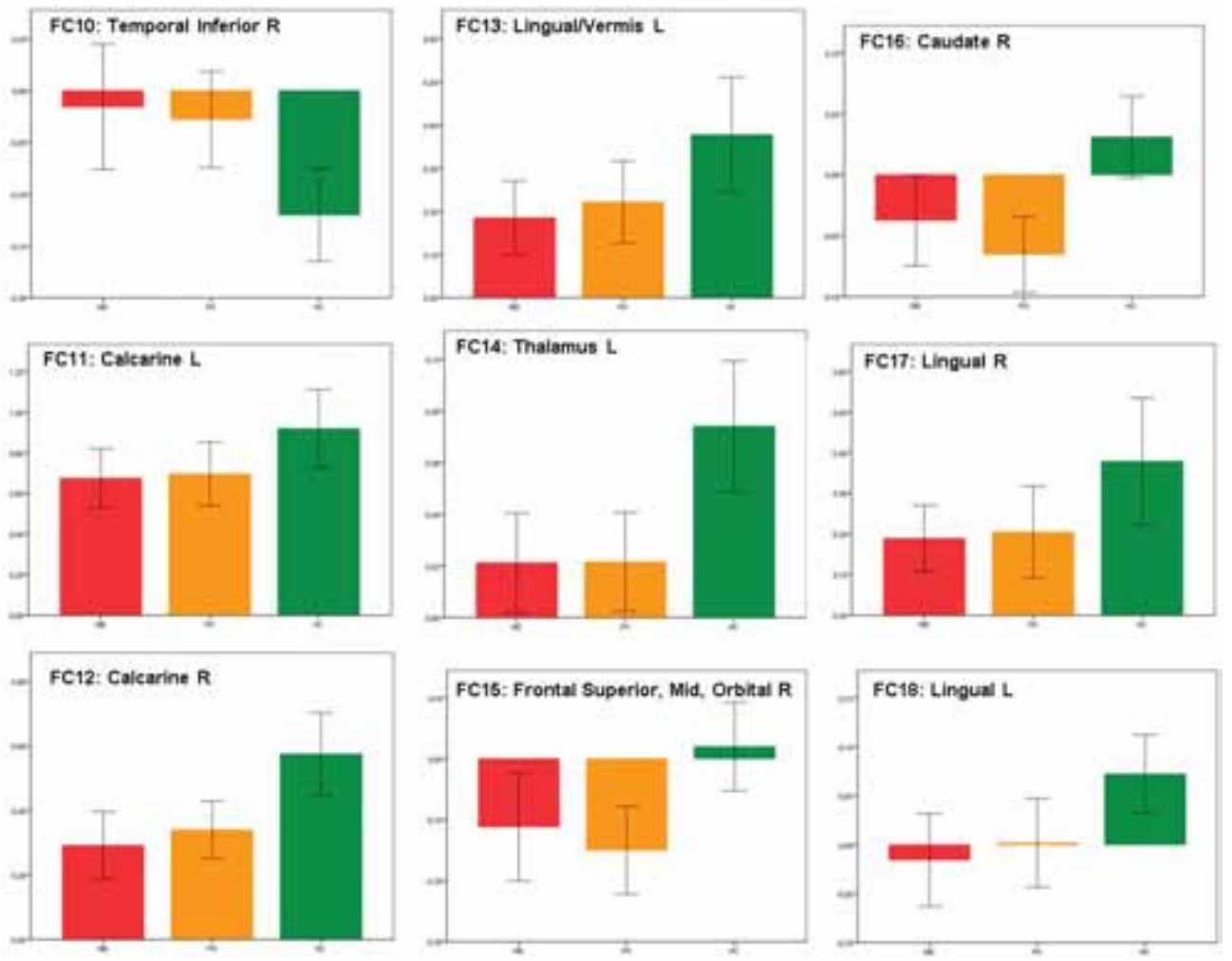

Supplementary Figure 2. Bar plots (error bars signify 95\% confidence intervals) for all effects. Red color bars correspond to the MD patient group, orange to the familial $M D$ vulnerability group and green to healthy controls. 

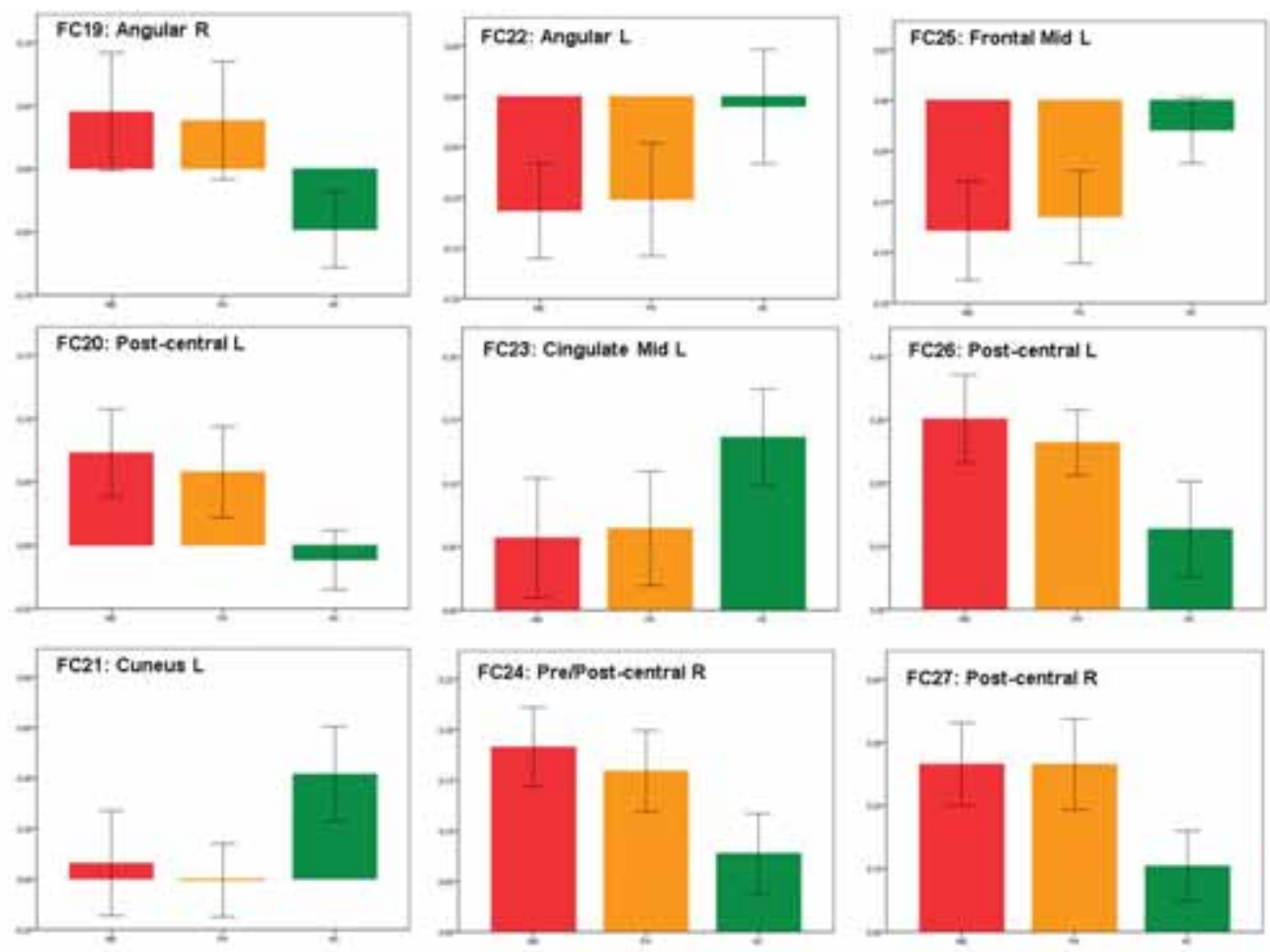

Supplementary Figure 3. Bar plots (error bars signify 95\% confidence intervals) for all effects. Red color bars correspond to the MD patient group, orange to the familial $M D$ vulnerability group and green to healthy controls. 


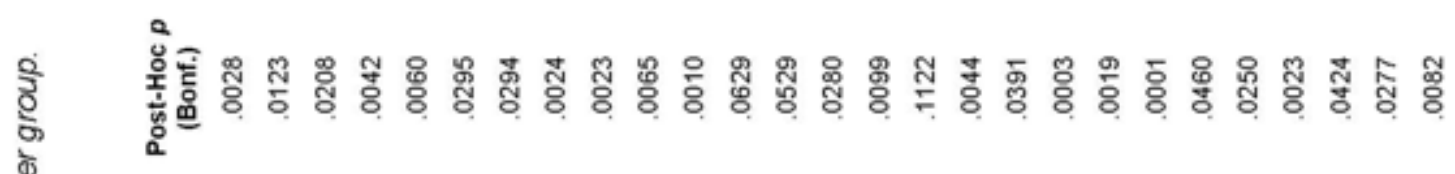

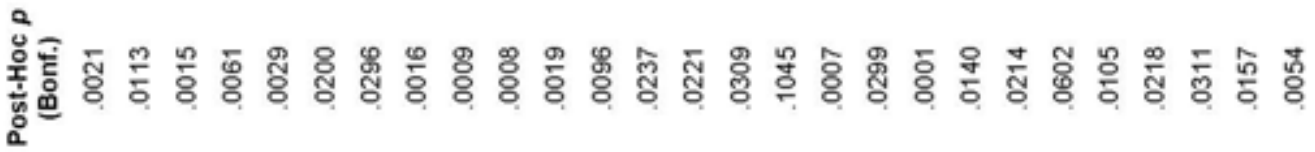

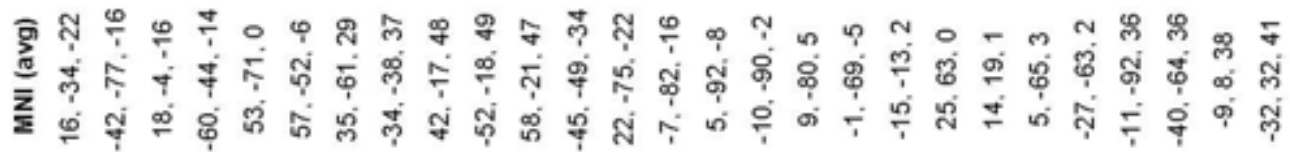

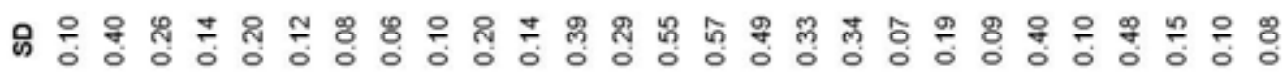
오

ॠ

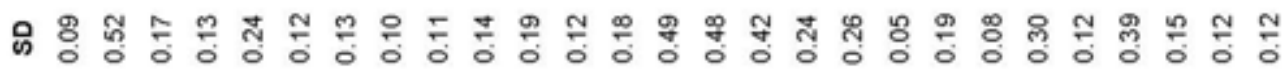
TI

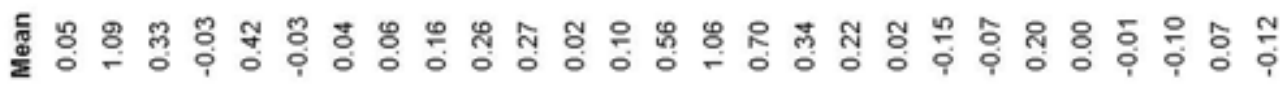

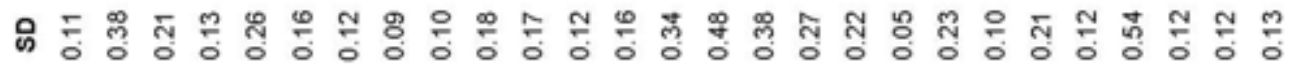
올

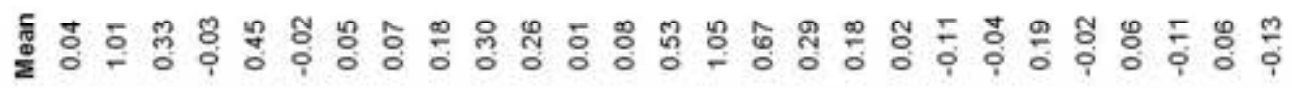

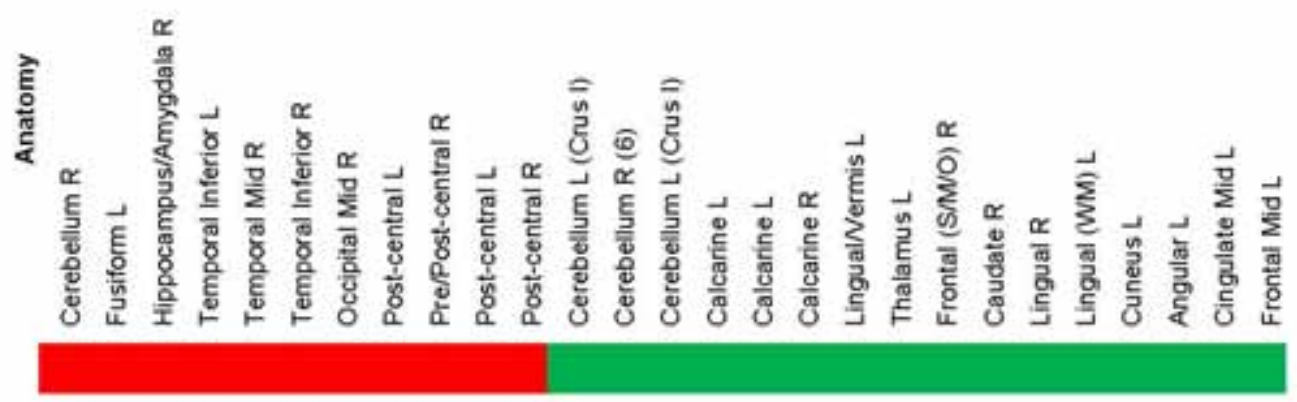


Anatomy labels correspond to AAL (Tzourio-Mazoyer et al., 2002); red corresponds to areas with increased BOLD signal in MD vulnerability while green corresponds to areas with decreased signal (see also main Figure 1). $P$ values in the penultimate column correspond to the comparison MD patient group versus healthy controls while $p$ values of last column to the comparison familial MD group versus healthy controls. 


\section{References}

Adolphs R, Spezio M. 2006. Role of the amygdala in processing visual social stimuli. Brain. 156:363-78.

Anderson IM, Del-Ben CM, Mckie S, Richardson P, Williams SR, Elliott R, Deakin JFW. 2007. Citalopram modulation of neuronal responses to aversive face emotions: a functional MRI study. Neuroreport. 18:1351-1355.

Anderson IM, Juhasz G, Thomas E, Downey D, McKie S, Deakin JFW, Elliott R. 2011. The effect of acute citalopram on face emotion processing in remitted depression: A pharmacoMRI study. Eur Neuropsychopharmacol. 21:140-148.

Anderson TR, Hu B, Iremonger K, Kiss ZHT. 2006. Selective attenuation of afferent synaptic transmission as a mechanism of thalamic deep brain stimulation-induced tremor arrest. J Neurosci. 26:841-850.

Apps M a J, Lockwood PL, Balsters JH. 2013. The role of the midcingulate cortex in monitoring others' decisions. Front Neurosci. 7:1-7.

Arnone D, McKie S, Elliott R, Thomas EJ, Downey D, Juhasz G, Williams SR, Deakin JFW, Anderson IM. 2012. Increased amygdala responses to sad but not fearful faces in major depression: Relation to mood state and pharmacological treatment. Am J Psychiatry. 169:841-850.

Arsalidou M, Barbeau EJ, Bayless SJ, Taylor MJ. 2010. Brain responses differ to faces of mothers and fathers. Brain Cogn. 74:47-51.

Azorin J.M., Benhaim P., Hasbroucq T., Possamai C.A. 1995. Stimulus preprocessing and response selection in depression: A reaction time study. Acta Psychol;89:95-100.

Barch DM, Gaffrey MS, Botteron KN, Belden AC, Luby JL. 2012. Functional brain activation to emotionally valenced faces in school-aged children with a history of preschool-onset major depression. Biol Psychiatry. 72:1035-1042.

Beck AT, Steer RA, Ball R, Ranieri W. 1996. Comparison of Beck Depression Inventories-IA and -II in psychiatric outpatients. J Pers Assess. 67:588-597.

Bhagwagar Z, Cowen PJ, Goodwin GM, Harmer CJ. 2004. Normalization of enhanced fear recognition by acute SSRI treatment in subjects with a previous history of depression. Am J Psychiatry. 161:166 -168.

Bistricky SL, Ingram RE, Atchley RA. 2011. Facial affect processing and depression susceptibility: Cognitive biases and cognitive neuroscience. Psychol Bull. 137: 9981028.

Bouhuys AL, Geerts E, Gordijn MC. 1999. Depressed patients' perceptions of facial emotions in depressed and remitted states are associated with relapse: A longitudinal study. Journal of Nervous and Mental Disease.187:595-602. 
Bourke C, Douglas K, Porter R. 2010. Processing of facial emotion expression in major depression: a review. Aust N Z J Psychiatry. 44: 681-96.

Buyukdura JS, McClintock SM, Croakin PE. 2010.Psychomotor retardation in depression: Biological underpinnings, measurement and treatment. Prog Neuropsychopharmacol Biol Psychiatry. 35:395-409.

Canli T, Congdon E, Todd Constable R, Lesch KP. 2008. Additive effects of serotonin transporter and tryptophan hydroxylase-2 gene variation on neural correlates of affective processing. Biol Psychol. 79:118-125.

Chan SWY, Norbury R, Goodwin GM, Harmer CJ. 2009. Risk for depression and neural responses to fearful facial expressions of emotion. Br J Psychiatry. 194:139-145.

Dannlowski U, Kersting A, Donges U, Lalee-Mentzel J, Arolt V, Suslow T. 2006. Subliminal facial affect priming is associated with therapy response in clinical depression. Eur Arch Psychiatry Clin Neurosci.256: 215-221.

Dannlowski U, Ohrmann P, Bauer J, Deckert J, Hohoff C, Kugel H, Arolt V, Heindel W, Kersting A, Baune BT, Suslow T. 2008. 5-HTTLPR biases amygdala activity in response to masked facial expressions in major depression. Neuropsychopharmacology. 33:418424.

Dannlowski U, Ohrmann P, Konrad C, Domschke K, Bauer J, Kugel H, Hohoff C, Scho S, Kersting A. 2009. Reduced amygdala - prefrontal coupling in major depression: association with MAOA genotype and illness severity. Int J Neuropsychopharmacol. 1122.

Del-Ben CM, Ferreira CAQ, Graeff FG. 2008. Serotonergic modulation of face-emotion recognition. Braz J Med Biol Research. 41:263-269.

Derogatis L, Melisaratos N. 1983. The Brief Symptom Inventory: An introductory report. Psychological Medicine. 13:595-605.

Domschke K, Braun M, Ohrmann P, Suslow T, Kugel H, Bauer J et al. 2006. Association of the functional -1019C/G 5-HT 1A polymorphism with prefrontal cortex and amygdala activation measured with 3T fMRI in panic disorder. Int J Neuropsychopharmacol. 9: 349-355.

Drevets WC, Videen TO, Price JL, Preskorn SH, Carmichael ST, Raichle ME. 1992. A functional anatomical study of unipolar depression. J Neurosci. 12:3628-41.

Elliott R, Zahn R, Deakin JF, Anderson IM. 2011. Affective cognition and its disruption in mood disorders. Neuropsychopharmacology. 36:153-182.

Ferrucci R, Giannicola G, Rosa M, Fumagalli M, Boggio PS, Hallett M, Zago S, Priori A. 2012. Cerebellum and processing of negative facial emotions: cerebellar transcranial DC stimulation specifically enhances the emotional recognition of facial anger and sadness. Cogn Emot. 26:786-799. 
Frodl T, Koutsouleris N, Bottlender R, Born C, Jäger M, Mörgenthaler M, Scheuerecker J, Zill P, Baghai T, Schüle C, Rupprecht R, Bondy B, Reiser M, Möller H-J, Meisenzahl EM. 2008. Reduced gray matter brain volumes are associated with variants of the serotonin transporter gene in major depression. Mol Psychiatry. 13:1093-1101.

Fu CHY, Williams SCR, Cleare AJ, Brammer M, Walsh ND, Kim J, Andrew CM, Pich EM, Williams PM, Reed LJ, Mitterschiffthaler MT, Suckling J, Bullmore ET: Attenuation of the neural response to sad faces in major depression by antidepressant treatment: a prospective, event-related functional magnetic resonance imaging study. Arch Gen Psychiatry 2004, 61:877-889.

Fu CHY, Mourao-Miranda J, Costafreda SG, Khanna A, Marquand AF, Williams SCR, Brammer MJ. 2008. Pattern classification of sad facial processing: toward the development of neurobiological markers in depression. Biol Psychiatry. 63:656-662.

Fusar-Poli P, Placentino A, Carletti F, Landi P, Allen P, Surguladze S, Benedetti F, Abbamonte M, Gasparotti R, Barale F, Perez J, McGuire P, Politi P. 2009. Functional atlas of emotional faces processing: a voxel-based meta-analysis of 105 functional magnetic resonance imaging studies. J Psychiatry Neurosci. 34:418-432.

Gaffrey MS, Luby JL, Belden AC, Hirshberg JS, Volsch J, Barch DM. 2011. Association between depression severity and amygdala reactivity during sad face viewing in depressed preschoolers: An fMRI study. J Affect Disord. 129:364-370.

Gohier B, Senior C, Radua J, El-Hage W, Reichenberg a., Proitsi P, et al. 2014. Genetic modulation of the response bias towards facial displays of anger and happiness. Eur Psychiatry. 29:197-202.

Gotlib IH, Krasnoperova E, Yue DN, Joormann J. 2004. Attentional biases for negative interpersonal stimuli in clinical depression. J Abnorm Psychol. 113:121 - 135.

Goulden N, McKie S, Thomas EJ, Downey D, Juhasz G, Williams SR, et al. 2012. Reversed frontotemporal connectivity during emotional face processing in remitted depression. Biol Psychiatry. 72: Society of Biological Psychiatry604-11.

Harmer CJ, O'Sullivan U, Favaron E, Massey-Chase R, Ayres R, Reinecke A, et al. 2009. Effect of acute antidepressant administration on negative affective bias in depressed patients. Am J Psychiatry. 166:1178-1184.

Haxby J, Hoffman E, Gobbini MI. 2000. The distributed human neural system for face perception. Trends Cogn Sci. 4:223-233.

Henderson SE, Vallejo AI, Ely B a., Kang G, Krain Roy A, Pine DS, Stern ER, Gabbay V. 2014. The neural correlates of emotional face-processing in adolescent depression: a dimensional approach focusing on anhedonia and illness severity. Psychiatry Res Neuroimaging. 224:234-241.

Homan P, Drevets WC, Hasler G. 2014. The effects of catecholamine depletion on the neural response to fearful faces in remitted depression. Int J Neuropsychopharmacol.17: 1419_ 1428. 
Homola GA, Jbabdi S, Beckmann CF, Bartsch AJ. 2012. A Brain Network Processing the Age of Faces. PLoS One. 7.

Hornboll B, Macoveanu J, Rowe J, Elliott R, Paulson OB, Siebner HR, Knudsen GM. 2013. Acute serotonin $2 \mathrm{~A}$ receptor blocking alters the processing of fearful faces in the orbitofrontal cortex and amygdala. J Psychopharmacol. 27:903-914.

lidaka T, Ozaki N, Matsumoto A, Nogawa J, Kinoshita Y, Suzuki T, et al. 2005. A variant $\mathrm{C} 178 \mathrm{~T}$ in the regulatory region of the serotonin receptor gene HTR3A modulates neural activation in the human amygdala. J Neurosci. 25: 6460-6466.

Joormann J, et al. 2007. Biased processing of emotional information in girls at risk for depression. Journal of Abnormal Psychology.116:135-143.

Joormann J, Gotlib IH. 2007. Selective attention to emotional faces following recovery from depression. J Abnorm Psychol. 116:80-85.

Keedwell PA, Andrew C, Williams SCR, Brammer MJ, Phillips ML. 2005. The neural correlates of anhedonia in major depressive disorder. Biol Psychiatry. 58:843-853.

Kesler-West ML, Kesler-West ML, Andersen a H, Andersen a H, Smith CD, Smith CD, Avison MJ, Avison MJ, Davis CE, Davis CE, Kryscio RJ, Kryscio RJ, Blonder LX, Blonder LX. 2001. Neural substrates of facial emotion processing using fMRI. Brain Res Cogn Brain Res. 11:213-226.

Kohler CG, Hoffman LJ, Eastman LB, Healey K, Moberg PJ. 2011. Facial emotion perception in depression and bipolar disorder: A quantitative review. Psychiatry Res.188:303-309.

Lau JYF, Goldman D, Buzas B, Fromm SJ, Guyer AE, Hodgkinson C, Monk CS, Nelson EE, Shen PH, Pine DS, Ernst M. 2009. Amygdala Function and 5-HTT Gene Variants in Adolescent Anxiety and Major Depressive Disorder. Biol Psychiatry. 65:349-355.

Lawrence NS, Williams AM, Surguladze S, Giampietro V, Brammer MJ, Andrew C, Frangou S, Ecker C, Phillips ML. 2004. Subcortical and ventral prefrontal cortical neural responses to facial expressions distinguish patients with bipolar disorder and major depression. Biol Psychiatry. 55:578-587.

Lee BT, Lee HY, Lee BC, Pae CU, Yoon BJ, Ryu SG, Choi IG, Lee MS, Ham BJ. 2009. Impact of the tryptophan hydroxylase 1 gene $\mathrm{A} 218 \mathrm{C}$ polymorphism on amygdala activity in response to affective facial stimuli in patients with major depressive disorder. Genes, Brain Behav. 8:512-518.

Leppanen JM, Milders M, Bell JS, Terriere E, Hietanen JK. 2004. Depression biases the recognition of emotionally neutral faces. Psychiatry Res.128:123 - 133.

Leppanen JM. 2006. Emotional information processing in mood disorders: A review of behavioral and neuroimaging findings. Curr Opin Psychiatry.19:34 -39. 
Leppänen JM, Nelson CA. 2009. Tuning the developing brain to social signals of emotions. Nat Rev Neurosci. 10:37-47.

Menon V, Glover GH, Pfefferbaum A. 1998. Differential activation of dorsal basal ganglia during externally and self paced sequences of arm movements. Neuroreport. 9(7):15671573.

Mingtian Z, Shuqiao Y, Xiongzhao Z, Jinyao Y, Xueling Z, Xiang W, Yingzi L, Jian L, Wei W. 2012. Elevated amygdala activity to negative faces in young adults with early onset major depressive disorder. Psychiatry Res - Neuroimaging. 201:107-112.

Miskowiak K, Glerup L, Vestbo C, Kessing L, Vinberg M. 2014. Aberrant neural and cognitive response to emotional faces stimuli in healthy monozygotic twins at heritable risk of depression. Eur Neuropsychopharmacol. 22: S195.

Monk CS, Klein RG, Telzer EH, Schroth EA, Mannuzza S, Moulton JL, et al. 2008. Amygdala and nucleus accumbens activation to emotional facial expressions in children and adolescents at risk for major depression. Am J Psychiatry. 165: 90-8.

Murrough JW, Collins KA, Fields J, DeWilde KE, Phillips ML, Mathew SJ, Wong E, Tang CY, Charney DS, losifescu D V. 2015. Regulation of neural responses to emotion perception by ketamine in individuals with treatment-resistant major depressive disorder. Transl Psychiatry. 5:e509.

Neumeister A, Drevets WC, Belfer I, Luckenbaugh DA, Henry S, Bonne O, Herscovitch P, Goldman D, Charney DS. 2006. Effects of a alpha 2C-adrenoreceptor gene polymorphism on neural responses to facial expressions in depression. Neuropsychopharmacology. 31:1750-1756.

Nomura EM, Rebe PJ. 2008. A review of medial temporal lobe and caudate contributions to visual category learning. Neuroscience and Biobehavioral Reviews. 32:279-291.

Pan LA, Hassel S, Segreti AM, Nau SA, Brent DA, Phillips ML. 2013. Differential patterns of activity and functional connectivity in emotion processing neural circuitry to angry and happy faces in adolescents with and without suicide attempt. Psychol Med. 43:2129_ 2142.

Raven J, Raven JC, Court JH. 2003. Manual for Raven's Progressive Matrices and Vocabulary Scales. Section 1: General Overview. San Antonio, TX: Harcourt Assessment.

Rawlings NB, Norbury R, Cowen PJ, Harmer CJ. 2010. A single dose of mirtazapine modulates neural responses to emotional faces in healthy people. Psychopharmacology (Berl). 212:625-634.

Ro T, Farne A, Johnson RM, Wedeen V, Chu Z, Wang ZJ, et al. 2007. Feeling sounds after a thalamic lesion. Annals of Neurology, 62:433-441, 2007.

Ro T. 2010. What can TMS tell us about visual awareness? Cortex. 46:110-113. 
Ruhé HG, Koster M, Booij J, van Herk M, Veltman DJ, Schene AH. 2014. Occupancy of serotonin transporters in the amygdala by paroxetine in association with attenuation of left amygdala activation by negative faces in major depressive disorder. Psychiatry Res Neuroimaging. 221:155-161.

Rush AJ, Trivedi MH, Ibrahim HM, Carmody TJ, Arnow B, Klein DN, Markowitz JC, Ninan PT, Kornstein S, Manber R, Thase ME, Kocsis JH, Keller MB. 2003. The 16-item Quick Inventory of Depressive Symptomatology (QIDS) Clinician Rating (QIDS-C) and SelfReport (QIDS-SR): A psychometric evaluation in patients with chronic major depression. Biological Psychiatry, 54:573-583.

Scheuerecker J, Meisenzahl EM, Koutsouleris N, Roesner M, Med C, Schöpf V, Linn J, Wiesmann M, Brückmann H, Möller H-J, Frodl T. 2010. Orbitofrontal volume reductions during emotion recognition in patients with major depression. J Psychiatry Neurosci. 35:311-321.

Sheline YI, Barch DM, Donnelly JM, Ollinger JM, Snyder AZ, Mintun MA. 2001. Increased amygdala response to masked emotional faces in depressed subjects resolves with antidepressant treatment: an fMRI study. Biol Psychiatry. 50:651-658.

Stănişor L, van der Togt C, Pennartz CM a, Roelfsema PR. 2013. A unified selection signal for attention and reward in primary visual cortex. Proc Natl Acad Sci U S A. 110:9136-9141.

Stuhrmann A, Dohm K, Kugel H, Zwanzger P, Redlich R, Grotegerd D, Rauch AV, Arolt V, Heindel W, Suslow T, Zwitserlood P, Dannlowski U. 2013. Mood-congruent amygdala responses to subliminally presented facial expressions in major depression: Associations with anhedonia. J Psychiatry Neurosci. 38:249-258.

Surguladze S, Brammer MJ, Keedwell P, Giampietro V, Young AW, Travis MJ, Williams SCR, Phillips ML. 2005. A differential pattern of neural response toward sad versus happy facial expressions in major depressive disorder. Biol Psychiatry. 57:201-209.

Suslow T, Konrad C, Kugel H, Rumstadt D, Zwitserlood P, Schöning S, Ohrmann P, Bauer J, Pyka M, Kersting A, Arolt V, Heindel W, Dannlowski U. 2010. Automatic moodcongruent amygdala responses to masked facial expressions in major depression. Biol Psychiatry. 67:155-160.

Thomas EJ, Elliott R, McKie S, Arnone D,DowneyD, Juhas ZG, et al. 2011. Interaction between a history of depression and rumination on neural response to emotional faces. Psychol Med. 41:1845-1855.

Tottenham N, Tanaka JW, Leon AC, McCarry T, Nurse M, Hare TA, et al. 2009. The NimStim set of facial expressions: Judgments from untrained research participants. Psychiatry Res.168: 242-249.

Tricomi E, Fiez JA. 2008. Feedback signals in the caudate reflect goal achievement on a declarative memory task. Neuroimage. 41:1154-1167.

Van den Bulk BG, Meens PHF, van Lang NDJ, de Voogd EL, van der Wee NJA, Rombouts SARB, Crone EA, Vermeiren RRJM. 2014. Amygdala activation during emotional face 
processing in adolescents with affective disorders: the role of underlying depression and anxiety symptoms. Front Hum Neurosci. 8:393.

Van der Veen FM, Evers EA T, Deutz NEP, Schmitt JAJ. 2007. Effects of acute tryptophan depletion on mood and facial emotion perception related brain activation and performance in healthy women with and without a family history of depression. Neuropsychopharmacology. 32: 216-224.

Victor TA, Furey ML, Fromm SJ, Ohman A, Drevets WC. 2010. Relationship between amygdala responses to masked faces and mood state and treatment in major depressive disorder. Arch Gen Psychiatry. 67:1128-1138.

Victor TA, Furey ML, Fromm SJ, Bellgowan PSF, Öhman A, Drevets WC. 2012. The Extended Functional Neuroanatomy of Emotional Processing Biases for Masked Faces in Major Depressive Disorder. PLoS One. 7:1-9.

Zeng L-L, Shen H, Liu L, Wang L, Li B, Fang P, Zhou Z, Li Y, Hu D. 2012. Identifying major depression using whole-brain functional connectivity: a multivariate pattern analysis. Brain. 135:1498-1507.

Zhang Y, Du G, Yang Y, Qin W, Li X, Zhang Q. 2015. Higher integrity of the motor and visual pathways in long-term video game players. Front Hum Neurosci. 9:1-7. 

Chapter 5

General Discussion 


\section{Thesis aims and main findings}

The objectives of the present thesis were on the one hand the development and application of parcellation methods to study the functional organization of extended cortical regions and on the other, the identification of functional connectivity and BOLD activation abnormalities that either precede or coincide with episodes of Major Depressive Disorder (MDD). We achieved these goals by studying a cohort of participants with MDD, individuals at MDD-risk and a healthy control group. We used a non-invasive technique of in vivo cortical mapping based on graph theory, low frequency BOLD co-fluctuations (i.e. MRI functional connectivity) during rest and BOLD activations during processing of affective face stimuli.

Recently developed MRI connectivity-based parcellations have made it possible to study the structural and functional organization of extended cortical regions systematically and in an unbiased way. This constitutes a significant step forward compared to seed-based analyses, where functional "units" of organization are usually inferred from task activations, or analyses utilizing general anatomical labels, which do not fully take into account what is known about cortical structure. Using low-frequency co-fluctuations in the BOLD signal during rest as input data, parcellation techniques reveal a sort of "default" or basic pattern of organization among regions, independent from experimental manipulations, task parameters or other demands. In our first study, described in Chapter 2, we extended the use of a novel parcellation method based on the intrinsic connectivity of voxels and the state-of-the-art Louvain modularity detection algorithm, to the study of the orbitomedial prefrontal cortex (OMPFC), an extended cortical region significant for functions of reward, emotion and decision making. The study revealed that the left hemisphere OMPFC is organized in 19 functional cortical fields in healthy controls. The way the fields were delineated replicated known cytoarchitectonic, and thus connectomic/functional, trends such as the cortical variation along two spatial gradients in the orbitofrontal cortex and the anterior versus mid-cingulate differentiation in the medial PFC. In addition to being neuroanatomically plausible, the delineated fields were found to be functionally organized in large-scale systems, in line with anatomical studies suggesting the existence of a medial and an orbital network of individual regions. Finally, our first study underscored the necessity of including signalto-noise measures of quality, replicability, and stability of functional connectivity metrics in MRI studies. This is important, especially for areas known to be susceptible to MRI artifacts, so that results are interpreted in the context of the ability to detect and replicate the effects under investigation.

One way to validate parcellation results is to compare the delineated fields of the parcellation map with existing cytoarchitectonic templates and tracing studies. The borders and absolute and relative spatial position of each field is compared to previous maps and homology is established qualitatively based on this information and on the similarity of connectivity 
patterns. To the extent that the parcellation fields truly represent organizational units, one expects that seeding from their center of mass will reveal differences between populations or manipulations. In our second study, described in Chapter 3, we used the functional fields of the left hemisphere parcellation map we created in order to systematically and in an unbiased way examine alterations of connectivity within the OMPFC, an area important for various psychiatric disorders including MDD. This study is the first illustration of how data-driven maps can aid the elucidation of dysfunction within extended cortical patches. The second aim of this study was to distinguish between connectivity abnormalities that precede the onset of the disease and are thus found both in patients and in at-risk individuals, versus those that coincide with the acute MDD phase. In the long run predisposing abnormalities can serve as endophenotypes, i.e. intermediate biological processes that can be linked to genotypes, whereas correlates of the depressed state can be used for diagnostics and predictors of treatment response. Our results suggest on the one hand that putative MDD endophenotypic aberrations found are functional connectivity abnormalities between the rostral cingulate and attentional regions, dorsal medial areas and occipital cortex (dorsal visual stream) and the anterior insula and the periaqueductal gray. On the other hand, MDD disease or acute phase markers are additional connectivity impairments between the rostral cingulate and the precuneus, between the anterior insula and striatal, fusiform and cerebellar regions, and between the dorsal cingulate and lateral orbitofrontal cortex and various cortical regions.

The functional connectivity markers we observed in our first study for MDD vulnerability and the MDD acute phase are resting-state patterns. We speculated that disturbance of these functional connections relates to the altered cognitive and affective processes seen in MDD (but also in other psychiatric disorders; Harvey et al., 2004), in the form of anticipatory affect, attentional and mood-congruent interpretation biases in the processing of incoming stimuli. Attentional and/or interpretational biases in processing of incoming visual stimuli are often studied with the use of facial stimuli with neutral or emotional expressions. Facial stimuli are carriers of complex socioemotionally highly relevant information; they depict agents with mental states and motivations which are vital to decode in order to ensure survival and personal well-being. At the same time, emotional expressions on human faces naturally activate in the observer the systems representing his/her own emotional states. Given these, it is not surprising that the presentation of facial stimuli is a simple yet effective paradigm to study affective modulation in MDD. Brain activation abnormalities during face processing have been repeatedly reported in MDD patients. However, it is not clear yet whether they are tied to the acute phase or characterize the MD vulnerability state as well, as our functional coupling results suggest. Our third study, described in Chapter 4, was designed to examine two hypotheses: first, whether the same 
group of patients showing the abnormal resting state connectivity patterns also show abnormal face processing in overlapping regions and second, whether abnormal face processing is present in the at-risk group as well. The findings of our last study, while they did not provide support for impaired activation of any of the OMPFC areas, revealed that a series of (other) regions with deviant BOLD responses found in MDD patients are also dysfunctional in the vulnerability state. These deviant responses follow a pattern of enhanced responsivity in limbic and ventral visual stream areas combined with diminished response in primary visual and frontal regions, as well as striatal and thalamic regions. Abnormal facial processing in these regions did not occur exclusively in the presence of intense emotional stimuli but was observed during processing of neutral faces as well. Our study results on face processing therefore suggest that abnormalities in affective modulation of environmental triggers might predispose at-risk individuals to perceive behaviorally relevant information, including faces and social interactions, in an affectively biased way and, thus, in this way be etiologically implicated in MDD.

\section{Mapping the functional organization of the human cortex}

Parcellation methods open the way to map the functional organization of the human brain and understand its function in health and disease. The methods developed in the context of this thesis are based on graph theory and functional connectivity, i.e. statistical significant co-fluctuation of the BOLD signal between regions. For comprehensive models of brain function however, it is important to be able to decipher whether communication is implemented via direct or indirect white matter pathways between pairs of brain regions as well as the direction of information flow. Thus, in the future such approaches need to be complemented with anatomical data, effective connectivity data, and laminar patterns of functional coupling as well. Even more importantly, future models of brain function in psychiatry need to be linked to molecular processes so that medicinal therapies can be improved. Therefore, to make clinically significant progress, brain organization models need to be complemented with information about neurotransmitter pathways as well. Integrating all these pieces of information increases the complexity of our models exponentially. We should thus in parallel develop sophisticated yet accessible ways to represent and simulate (e.g. computational models) the function of structural and functional directed neural networks for any real understanding of their purpose. The EU-funded human brain mapping project is a first step towards generating this level of information integration. Finally, it should be pointed out that in vivo mapping with high-field MRI and multimodal brain imaging will likely uncover additional levels of functional organization and will certainly modify our current paradigms.

The parcellation method used in the context of this thesis holds promise for the development of individually optimized clinical applications. 
The Louvain modularity detection algorithm we employed in our first study is able to delineate functional cortical fields at the level of the individual subject. These fields are subsequently merged/combined in a group representation of the cortical organization. Two points are worth mentioning with regard to this approach. Firstly, parcellation studies are currently in their infancy and, although promising, their results warrant replication in order to achieve consensus on some basic features of cortical topology. The example of early cytoarchitectonic maps is telling. Inadequate sampling, that is, group averaging of non-representative samples leads to results that do not replicate well nor generalize. We should thus strive to systematically explore the range of inter-individual variability in brain organization in order to create useful enough maps. Employment of large enough samples $(\mathrm{N}>1000)$ and validation of network maps in several samples, already employed by some groups (Yeo et al., 2011), should be regarded as a necessity rather than luxury.

The second point of concern our in vivo parcellation approach relates to the individually delineated fields. The centers of mass of the individual fields could be used as targets to guide individually optimized diagnosis and treatment in psychiatric practice. We should aim for a future, in which a 5 minute resting state scan, with no need of functional localizers or mental manipulations that depend heavily on the patient's compliance and motivation, would provide the targets for our neuro-modulation therapies. In order to create that future we need to understand better some parameters of our techniques. As discussed briefly at the end of the first chapter, particular methodological choices influence our results in ways that we cannot yet fully control. One such example is the choice of threshold for the binarization of the correlation matrix before its partitioning by the Louvain algorithm. The strength of the correlations in each individual dataset and in certain cortical areas varies and this has an influence on the resulting parcellation, since the applied threshold is inversely correlated with the number of delineated fields. During the research carried out in the context of the current thesis we stumbled upon this issue. In our first study, we used as seeds for the wholebrain connectivity profiles the centers of mass of the delineated fields at the individual level (i.e. modules) instead of the cluster coordinates (i.e. group weighted average). This was also our initial aim in the second study, in which we compare whole-brain connectivity profiles across groups. However, using the individually optimized seeds of the modules that correspond to a cluster, instead of the average coordinates of each cluster, led to a design with missing observations, since not all participants have modules corresponding to every cluster (see methods of Chapter 2). The results from such a design, aside from a loss in power, are difficult to interpret when one wants to draw conclusions across the repeated-measures comparisons. Since we currently lack objective ways to evaluate and address the impact of the choice of threshold on the individual parcellation maps and how results of individual 
datasets relate to one another, it was not possible to use the optimized seeds. While the average coms still lead to meaningful results, our ability to detect clinically significant differences or more differences might be enhanced if we had managed to capture better the individual functional OMPFC organization. This means that we need to handle this issue in order to be able to provide individually optimized targets for treatment applications.

\section{Neuroimaging markers of MD}

In our patient studies of Chapters 3 and 4 we sought to isolate abnormalities in functional connectivity and BOLD activation that characterize the genetic MDD vulnerability. Operationalizations of variables in research are often guided by pragmatic considerations such as financial means, time constraints and other issues (i.e. availability of sample, compliance, motivation to participate, etc.). We operationalized genetic vulnerability as having a first degree blood relative with MDD. However, pooling a sample with no genotyping does not ensure that all relatives carry the polymorphisms conferring vulnerability. Further, for reasons mentioned in the introduction (Chapter 1), in many cases of familial vulnerability (particularly in those of offspring of MDD patients) genetic load and environmental adversity is present. Thus, ideally, vulnerability should be measured in the same samples both by genotyping and by quantifying parameters such as childhood trauma/adversity, quality of attachment in primary relationships, psychological and societal/financial impact of parental MDD, socio-economic circumstances, and important life events as well. The above notwithstanding, the MDD vulnerability FC and BOLD changes we report are an important first step in the identification of biomarkers. These changes represent potential regions of interest in genetic studies that can help establish links from the genetic and molecular levels to brain processes and ultimately behavior and should be pursued further.

In the patient studies described here we took the approach of conjunction analyses in which we dissociated effects that are full-blown abnormalities in both at-risk individuals and MDD patients. This might give the impression that vulnerability is characterized by a few focal changes. However, the results we report here are only part of the differences between the MDD groups and controls. In exploratory analyses (not reported) we have also looked at the gradual changes associated with MDD. Those results, similar to the markers, confirm the presence of extensive connectivity abnormalities with basic sensory areas and the OMPFC. Altered connectivity patterns of prefrontal areas with sensory systems such as the ones we report in the current thesis, as well as differential processing of facial stimuli, signify that MDD entails a fundamentally different way of seeing the physical and social world. It is possible that functional connectivity changes that contribute to enhanced threat reactivity coupled with altered top-down mechanisms of attending to and making sense of the environment set the stage for a 
fundamentally different experience already in MDD vulnerability. These differences if coupled with high environmental demands or adversity, might lead to the changes that give rise to depressive symptoms.

Although such speculations are very interesting, it remains a challenge to give meaning to the connections affected by MDD. In future studies we should pursue ways to systematically test how functional connectivity disturbances relate to specific behavioral and mental processes we know are altered in MDD (i.e. attentional, interpretational and reasoning biases, impaired top-down control, inflexibility in choosing defense/stress regulation strategies, reality and arousal intolerant systems, etc.). Although it is not clear yet exactly how such processes map on the self-reported symptoms, they are nonetheless a sort of "endophenotypes" on their own, since they can be objectively measured and quantified; thus they can significantly aid empirical studies compared to self-reports.

\section{From biological findings to biomarkers}

Neuroscience research in psychiatric disorders has a specific and measurable objective: to produce results that work in clinical practice. The neuroimaging results reported here although valuable are only a tiny step towards future clinical applications. To achieve clinical relevance and applicability, a number of issues need to be addressed. First, psychiatric disorders exist in a continuum from idiosyncratic characteristics to dysfunctional states to psychiatric conditions. Given that, demonstrating differences in extreme comparisons between "picture-perfect healthy controls and prototypical patients" (Kapur et al., 2012) will not easily translate into robust classifications of subtle and continuous differences in the real world. Second, our DSM classifications are artificial dichotomies not only between "health" and "disease" but also among disorders since extensive comorbidity is the rule rather than the exception in clinical practice. Attempts to identify "trans-diagnostic" processes which cut across the current DSM taxonomy (Harvey et al., 2004) and inform our understanding of the discreetness and overlap among disorders need to be extended to neuroimaging results (Buckholtz and Meyer-Lindenberg, 2012). Lastly, MDD, like most syndromes, is highly heterogeneous. The findings reported here are average differences between groups. This means that not all members of the different groups show all changes reported at the group level. While various factors might account for this, one aspect of the phenomenon relates to the existence of subtypes of patients. The identification of subtypes within MDD will help us move away from diverse, sample-specific findings towards concrete and more replicable results and will open the way for personalized/stratified medicine and individually optimized treatments. 


\section{References}

Buckholtz JW, Meyer-Lindenberg A (2012): Psychopathology and the human connectome: toward a transdiagnostic model of risk for mental illness. Neuron 74: $990-1004$.

Harvey, A. G., Watkins, E., Mansell, W., \& Shafran, R. (2004). Cognitive behavioural processes across psychological disorders: A transdiagnostic approach to research and treatment. Oxford: Oxford University Press.

Kapur S, Phillips AG, Insel TR (2012): Why has it taken so long for biological psychiatry to develop clinical tests and what to do about it?: Molecular Psychiatry 17: 1174-1179.

Yeo BT, Krienen FM, Sepulcre J, Sabuncu MR, Lashkari D, Hollinshead M, Roffman JL, Smoller JW, Zöllei L, Polimeni JR, Fischl B, Liu H, Buckner RL. 2011. The organization of the human cerebral cortex estimated by functional connectivity. J Neurophysiol. 106:1125-1165. 
Major Depressive Disorder (MDD) is a psychiatric syndrome characterized by various affective, cognitive and somatic symptoms which cause significant loss of functioning and distress in patients. MDD is multifactorial and among other factors, genetic load influences the development of the syndrome. Immediate (first-degree blood) relatives of MDD patients are at increased risk of developing MDD themselves. Neurobiologically, MDD is a connectivity disorder in which the functional communication within an extended cortical region, the orbitomedial prefrontal cortex (OMPFC) is disturbed. It is also a disorder characterized by abnormalities in affective processing regions, the function of which can be effectively studied using facial emotional expressions as stimuli. Early diagnosis and individually tailored treatment is instrumental for the alleviation of MDD. In order to improve current prevention, diagnosis and treatment strategies, it is important to discriminate between predisposing factors, precipitating conditions, manifestations of the current depressive episode and possible adaptation-to-disease or compensation processes. By studying large cohorts of patients, at-risk individuals and healthy controls, we can distinguish brain imaging MDD vulnerability markers (i.e. objectively measured biological processes present at both patients and at-risk individuals which precede the onset of the disease state) and MDD disease markers (i.e. objectively measured biological processes observed solely during the acute phase).

The aims of the current thesis were threefold: a) validate and extend data-driven methods based on MRI functional connectivity and graph theory to parcellate the cortex, b) apply these methods to systematically and in an unbiased way delineate functional connectivity markers of MDD vulnerability and MDD disease within an extended cortical region and c) identify trait biomarkers of BOLD changes during face processing in MDD. We achieved these goals by testing a cohort of participants with Major Depressive Disorder, first-degree family relatives of MDD patients and healthy controls. We used a non-invasive technique of in vivo cortical mapping based on MRI connectivity, as well as low frequency BOLD co-fluctuations during rest and BOLD activations during processing of affective face stimuli.

Chapter 1 introduces the basic concepts and tools used in the research described in the current dissertation. Some clinical and theoretical background of MDD is also presented together with the aims and outline of the thesis.

Chapter 2 describes the first study in which a novel parcellation method based on the intrinsic connectivity of voxels and the state-of-the-art Louvain modularity detection algorithm was applied to the study of the OMPFC, a cortical region significant for functions of reward, emotion and decision making. The study revealed that the left hemisphere OMPFC is 
organized in 19 functional cortical fields in healthy controls. The way the fields were delineated replicated known cytoarchitectonic trends such as the cortical variation along two spatial gradients in the orbitofrontal cortex and the anterior versus mid-cingulate differentiation in the medial PFC. In addition to being neuroanatomically plausible, the delineated fields of the functional connectivity-based parcellation map were shown with agglomerative hierarchical clustering to be organized in large-scale systems in line with anatomical studies suggesting the existence of a medial and an orbital network of individual regions. Finally, this study underscored the usefulness of including measures of MRI quality, replicability (across runs and hemispheres) and inter-run stability of functional connectivity metrics in neuroimaging studies.

Chapter 3 is concerned with the application of the map developed in the first study to psychiatric disorders. It details the second study of this dissertation in which we used the left-hemisphere parcellation map mentioned above in order to systematically and in an unbiased way examine alterations of connectivity within the OMPFC, an area important for MDD. This study is the first illustration of how data-driven delineated maps can aid the elucidation of dysfunction in entire cortical patches and the first to distinguish between OMPFC connectivity abnormalities that precede the onset of the disease versus those that are present only during the acute MDD phase. The results of this study highlighted that the rostral cingulate and the anterior insula should be the focus of investigation for endophenotypes of vulnerability and biomarkers of the acute MDD phase.

Chapter 4 presents the last study of the thesis in which we examined which of the various abnormalities in BOLD activation during face processing previously reported for the MDD acute phase also characterize the MDD vulnerability state. The results underscored that a series of regions with deviant BOLD responses found in MDD patients are also dysfunctional in the vulnerability state. These deviant responses follow a pattern of enhanced reactivity in limbic and lateral visual areas combined with diminished response in primary visual, striatal, thalamic and frontal regions. Abnormal facial processing in these regions does not occur exclusively in the presence of intense emotional stimuli but is uniform during processing of faces irrespective of valence. These findings suggest that abnormalities in general face processing might predispose at-risk individuals to perceive social interactions in a distorted way and might be, thus, etiologically implicated in MDD.

Finally, Chapter 5 summarizes the main conclusions drawn in the context of this thesis and discusses the implications of our findings for parcellation approaches and neuroscience applications in psychiatry. The discussion is extended by considering open issues and future directions. 


\section{Knowledge Valorization}

Scientific knowledge is neither pursued nor produced in a vacuum as a theoretical or intellectual goal. It is meant to be utilized for societal benefit and progress. The valorization of scientific knowledge, i.e. its utilization in practice should therefore be actively explored and promoted. Scientific knowledge can be put to use in academic settings for research (science and methods) and educational purposes. Outside the university, experts in the public and private sector, institutions, individual consumers and the public in general benefit from the applied knowledge in the form of products and services. The present addendum discusses the valorization possibilities of the knowledge produced during this $\mathrm{PhD}$ project.

\section{Social \& economic relevance of current results}

MDD is a debilitating disorder which, according to the World Health Organization, is the leading cause of disability and is projected to be the first overall contributor to the worldwide burden of disease in 2030. MDD affects 350 million people globally and is associated with significant personal, societal and financial costs. These numbers become even more alarming if we take into account all affective disorders which involve episodes of depression as well as somatic conditions to which depressive episodes are secondary. MDD is among the leading causes of death as well, associated with suicide, death due to substance abuse and addiction or due to nonconformity of the patient to therapy in case of somatic disorders. The accurate, timely and effective prevention, diagnosis and treatment of MDD will essentially promote the physical and mental health and well-being of a significant percent of the population and at the same time reduce the economic burden in modern societies. Therefore, the valorization of knowledge towards these goals is very relevant both socially and economically.

In this work, we first extended and validated a new methodological approach to study the functional organization of the human orbitomedial prefrontal cortex. Complex cortical functional organization and connectivity is believed to subserve all higher human affective and cognitive faculties.

Subsequently, we used this and other methods to elucidate the role of certain cortical functional fields in MDD and further investigate abnormalities related to affective facial processing. The results of our studies hold promise for the development of applications to diagnose, subtype and treat MDD patients more effectively. These applications will enable clinicians to complement their diagnosis and decision making with neuroimaging/biological evidence about abnormal network function and reactivity. In this way, diagnosis and treatment strategies will become more effective in identifying patients in need of specific treatments. What is more, our results on the existence of network 
and reactivity pathology in genetically susceptible individuals might contribute to the development of psycho-prophylactic interventions.

\section{Target groups}

The results of the current thesis are immediately relevant to other researchers in the field who study functional organization, connectivity and reactivity in MDD disease and vulnerability states. Our results are also relevant to neuroscientists who are interested in parcellation methods and the organization and connectivity of the orbitomedial prefrontal cortex. Further, our results and their implications are of interest to health care professionals who diagnose and treat MDD patients. Most importantly, although not in the immediate future, our results will hopefully become highly relevant for patients, people at risk for depression and their families by contributing to the development of evidence-based diagnosis and treatment methods.

\section{Activities \& Products}

In the context of scientific research, our results give rise to new hypotheses to be tested mainly regarding the function and connectivity of the various cortical fields examined. With regard to the valorization of this knowledge outside of academia, resting state scans could be in the future used as adjunct means of diagnosis and treatment selection. Information on the cortical functional organization and functional connectivity of depressed patients' will assist health care professionals and clinicians in their diagnosis, prognosis and treatment plan. Information on affective processing or BOLD reactivity of specific cortical areas can also be used with the aid of machine learning algorithms to classify patients and at risk individuals. Since at present no single modality of neuroimaging findings sufficiently captures psychiatric pathology, it is also possible that algorithms that take into account both these pieces and other information will be used to classify and subtype depressive pathology. Finally, it is highly likely that soon resting state scans will not only assist in the selection of the best treatment option for individuals out of the existing ones but will also consistently provide new, individualized targets for connectivity-based therapies.

\section{Innovation}

The results presented in this thesis are innovative in following regards: firstly, we applied an innovative technique to parcellate the cortex into functional fields at the individual level. Although many methodological issues remain to be resolved, this parcellation approach might open the way for the study of cortical organization at the individual level, and many real-life applications in health and disease states. Secondly, we used large samples of depressed patients, healthy controls and people at genetic risk for depression, employing a biomarkers analysis. Our studies are innovative to 
the extent that for the first time we show that some of the most well established affective face processing abnormalities and connectivity aberrations are found already at the MDD vulnerable state. Though probably not all, some of these changes are etiologically linked to the development of depressive episodes and might be proven useful for applications in the field of prevention.

\section{Schedule \& Implementation}

The work presented here aimed to advance methods of delineating cortical functional organization based on connectivity and apply them to diseases known to be connectivity disorders and specifically MDD. What is more, we aimed to study and elaborate on brain changes not only in disease states but in vulnerability as well. Although our results will hopefully contribute to practical applications in the fields of prevention, diagnosis and personalized treatment in the future, there remain a number of critical issues to be addressed before the implementation of our knowledge valorization can be scheduled. These issues can be categorized as follows: those that have to do with the methodological aspects of our techniques and those that relate to the approach we have adopted within the psychiatry field. With regard to the methodological aspects, issues such as the establishment of the appropriate threshold for each dataset, cortical region and individual, the improvement of the group clustering/average parcellation solution and the elucidation of the function of each cortical field should be resolved. With regard to using these and other similar methods in clinical practice, issues to be addressed are the establishment of connectivity population norms, subtypes of depression, real-life samples and integration of information from multiple sources such as the connectivity and (re)activity information presented in the third and fourth chapters. 


\section{Curriculum Vitae \& Publications}

\section{Curriculum Vitae}

Zoe Samara was born in Thessaloniki on the $16^{\text {th }}$ of June 1977. In 1995 she ranked $5^{\text {th }}$ in the countrywide PanHellenic exams for a place in the Psychology Bachelor program at the Aristotle University. During her second year, she spent 9 months as an exchange student with the Erasmus scholarship at the University of Reading, UK. She wrote her Bachelor thesis on the paradigm of Connectionism in cognitive psychology. After graduation Zoe was trained as a psychodynamic psychotherapist for adults before moving to Leiden, NL in 2007. At Leiden University Zoe was granted the Leids Universiteits Fonds scholarship to follow the research MSc. program (cum laude) in Clinical and Health psychology. Her thesis research examined the neurobiological basis of a basic component of EMDR therapy. Zoe decided to conduct her $\mathrm{PhD}$ research at Maastricht University where she moved in 2010. Currently she is a post-doc at Stanford University in Palo Alto, California.

\section{Publications}

Samara Z., Evers E.A.T, Rajkowska G., Ramaekers J.G. \& Stiers P. (in preparation). Altered face processing in Major Depression Vulnerability: Inhabiting different social worlds?

Samara Z., Evers E.A.T., Goulas A., Uylings H.B.M., Rajkowska G., Ramaekers J.G. \& Stiers P. (in preparation). Orbitomedial prefrontal cortex in major depression: functional connectivity biomarkers of vulnerability and disease.

Samara Z., Evers E.A.T., Goulas A., Uylings H.B.M., Rajkowska G., Ramaekers J.G. \& Stiers P. (submitted). Human orbitomedial prefrontal cortex: Intrinsic connectivity parcellation and its functional organization.

Nieuwenhuis S., Elzinga B.M., Ras P.H., Berends F., Duijs P., Samara Z., \& Slagter H.A. (2013). Bilateral saccadic eye movements and tactile stimulation, but not auditory stimulation, enhance memory retrieval. Brain and cognition, 81(1), 52-6.

Samara Z., Elzinga B.M., Slagter H.A. \& Nieuwenhuis S. (2011). Do horizontal saccadic eye movements increase interhemispheric coherence? Investigation of a hypothesized neural mechanism underlying EMDR. Front. Psychiatry 2:4. 


\section{Conference abstracts}

Samara Z., Evers E.A.T, Uylings H.B.M., Rajkowska G., Ramaekers J.G. \& Stiers P. (2014). Can graph-theory parcellation schemes based on resting state functional connectivity inform us about depression disease and vulnerability status? Brain Connectivity, 4(9):P93A.

Samara Z., Evers E.A.T., Goulas A., Uylings H.B.M., Ramaekers J.G. \& Stiers P. (2013). The intrinsic organization of the human orbito-medial prefrontal cortex and the functional role of its subdivisions. Program No. 481.18/KKK26 Neuroscience Meeting Planner. San Diego, CA: Society for Neuroscience, 2013. Online.

Samara Z., Evers E.A.T., Huibers M., Peeters F., Ramaekers J.G. \& Stiers P. (2012). Is default mode network functional connectivity a biomarker of vulnerability to depression? Brain Connectivity, 2(4): A1-A156.

Stiers P., Goulas A., Samara Z. \& Uylings H.B.M. (2012). Intrinsic functional connectivity parcellation and task activation in human lateral prefrontal cortex. Brain Connectivity, 2(4): A1-A156.

Nieuwenhuis S., Elzinga B.M., Samara Z. \& Slagter H.A. (2011). Why saccades aid memory. 13th Winter Conference of the Dutch Psychonomic Society, Dec 16-17; Egmond aan Zee, NL.

Samara Z., Evers E.A.T., Huibers M., Peeters F., Ramaekers J.G. \& Stiers P. (2011). Neurobiological markers of Major Depressive Disorder, NWO 'Brain and Cognition: Societal innovation', Dec 1; Utrecht, Netherlands. 


\section{Acknowledgements}

The good life is one guided by knowledge and inspired by love (Bertrand Russell)

This thesis wouldn't have been written without the people who participated in our study. Especially our MD and FH groups, people who despite the pain they go though, each on their own way, came to help. Thank you for getting into that tiny space with the horrible noise and staying there; thank you for sharing your life stories and feelings. I wish there was a way to give you back answers and solutions at the moment instead of hopes and wishes for the future.

Peter, Lisbeth and Jan, thank you for giving me the opportunity to work with you on this specific project. It turned out to be way more difficult but also way more interesting than I could ever imagine. An old wisdom extract says "you don't always get the supervisors you want but you surely get the ones you need". I was lucky to have gotten a great mix of freedom to explore, guidance, inspiration, challenge and encouragement to pursue my goals. Peter, thank you for inspiring me never to lose sight of the heart of science and for showing me that one doesn't need much to create a lot.

Anke and Lisbeth, thank you for welcoming me to office 2.741 and helping me feel at home, thank you for all the laughs, tears, thoughts and chocolate we shared. Lisbeth, looking back at my time in Maastricht, I realize I have shared with and because of you some of the nicest experiences: critical thinking about our project and our goals as scientists, mud and sweat during Caveman run, truths and feelings at the book-club meetings, giggles and fun on train rides. Thank you for being there, things just wouldn't have been the same without you.

To all my colleagues at the NP\&PP department, thanks for the relaxed atmosphere, the fun outings and the supportive environment. A special, huge and fluffy thanks to Arjan for the movie nights, the jokes (well..some of them : ) and his help to PhD students. Jens, thank you for being my paranymph, the fun moments and the exciting discussions on our common topic; it's a pity there was such little time for those!

This thesis is dedicated to Phillip, my family and especially my mom, Elli. Mom, from all the gifts you have given me what I treasure the most is your faith and courage to fight forward regardless of the circumstances, and your insatiable passion for knowledge and understanding. Without this gift and your love I wouldn't have made it. 